Aus der Poliklinik für Zahnärztliche Prothetik

(Prof. Dr. med. dent. R. Bürgers)

im Zentrum Zahn-, Mund- und Kieferheilkunde

der Medizinischen Fakultät der Universität Göttingen

\title{
Untersuchung zur Reliabilität elektronischer Registriersysteme am Beispiel des ARCUSdigma II
}

\author{
INAUGURAL-DISSERTATION \\ zur Erlangung des Doktorgrades \\ der Zahnmedizin \\ der Medizinischen Fakultät der \\ Georg-August-Universität zu Göttingen
}

vorgelegt von

Aboud Rawik

aus

Aleppo / Syrien

Göttingen 2021 
Dekan:

Referent/in

Ko-Referent/in:

Drittreferent/in:
Prof. Dr. med. W. Brück

Prof. Dr. med. dent. R. Bürgers

PD Dr. Dr. P. Kauffmann

Prof. Dr. M. Oppermann

Datum der mündlichen Prüfung: 22.06.2021 
Hiermit erkläre ich, die Dissertation mit dem Titel "Untersuchung zur Reliabilität elektronischer Registriersysteme am Beispiel des ARCUSdigma II" eigenständig angefertigt und keine anderen als die von mir angegebenen Quellen und Hilfsmittel verwendet zu haben.

Göttingen, den 01.02.2021 
Die Daten, auf denen die vorliegende Arbeit basiert, wurden teilweise publiziert:

Krohn S, Rawik A, Rasing H, Kubein-Meesenburg D, Hampe T, Bürgers R (2018): The reliability of two different paraocclusal mounting methods for the digital instrumental functional diagnostics with ARCUSdigma II. J CranioMand Func 10, 355-363 


\section{Inhaltsverzeichnis}

Inhaltsverzeichnis ........................................................................................................

Abbildungsverzeichnis..............................................................................................................II

Tabellenverzeichnis ...........................................................................................

Abkürzungsverzeichnis .............................................................................................. V

$1 \quad$ Einleitung ............................................................................................................... 1

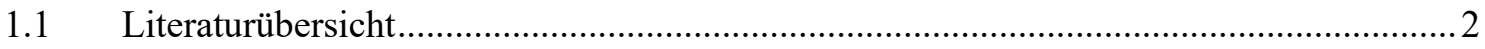

1.1.1 Die Anatomie des Kiefergelenks .................................................................................

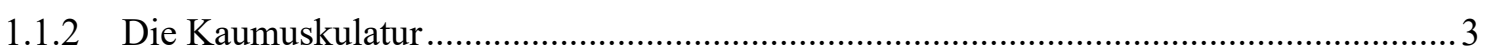

1.1.3 Mobilität des Unterkiefers .........................................................................................

1.1.4 Instrumentelle Bewegungsanalyse......................................................................... 14

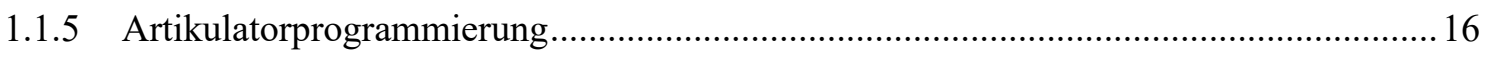

1.1.6 Elektronische Systeme zur Unterkieferaufzeichnung ...................................................21

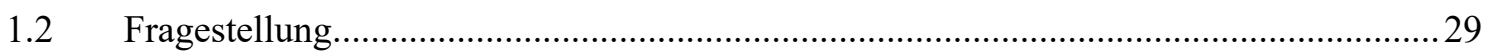

$2 \quad$ Material und Methoden ....................................................................................... 30

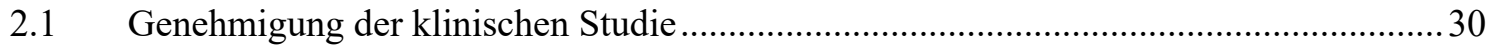

2.2 ProbandInnen und Ausschlußkriterien ................................................................... 30

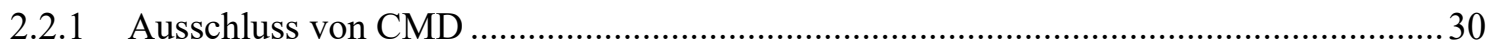

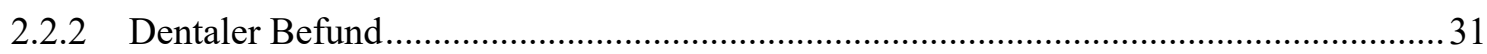

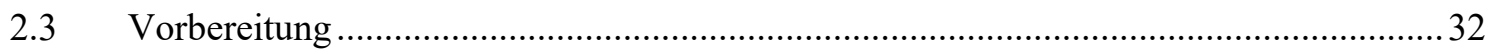

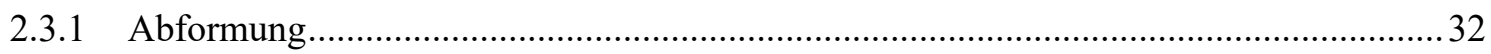

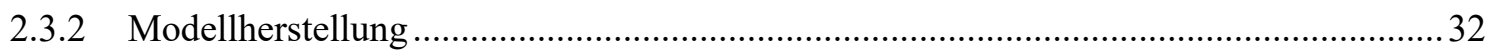

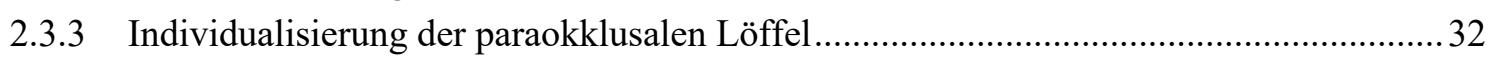

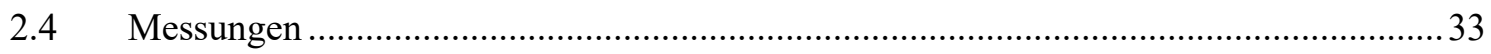

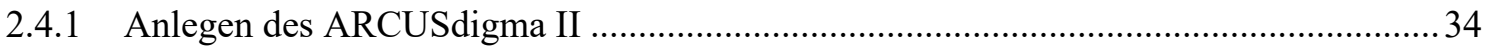

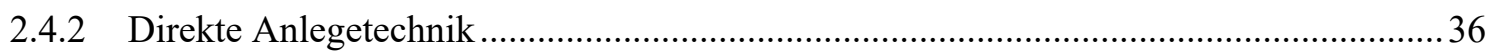

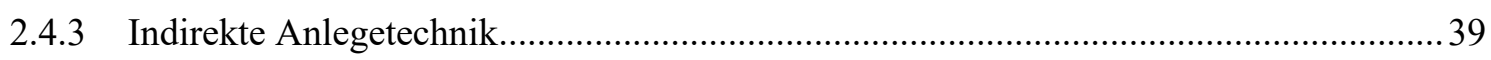

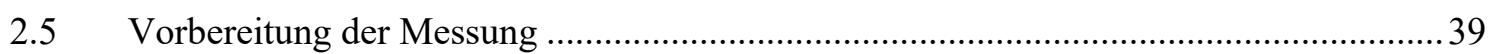

2.5.1 Kalibrierung des ARCUSdigma II.............................................................................. 40

2.5.2 Ablauf der Messungen .................................................................................... 40

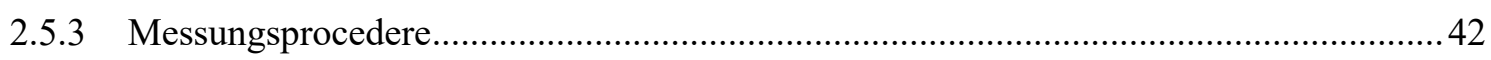

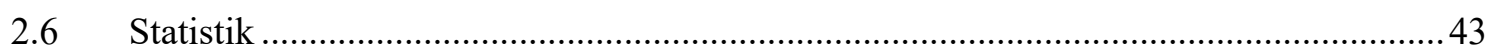

$3 \quad$ Ergebnisse .................................................................................................. 45

3.1 Erläuterungen zu den Grafiken ........................................................................ 45

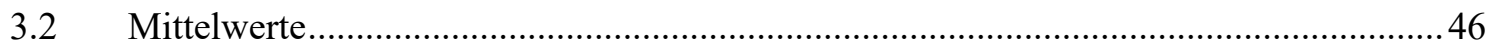




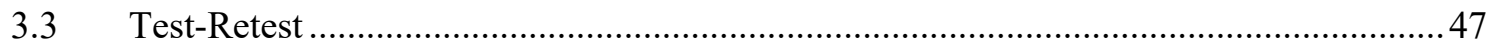

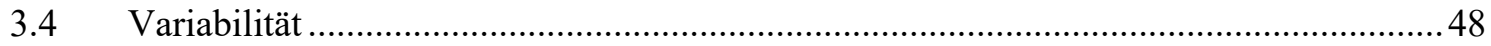

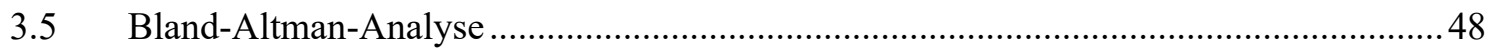

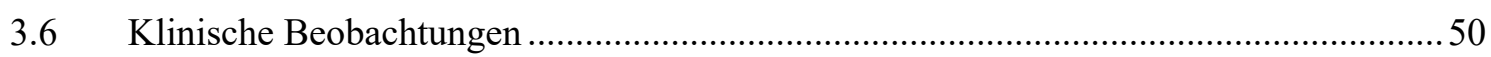

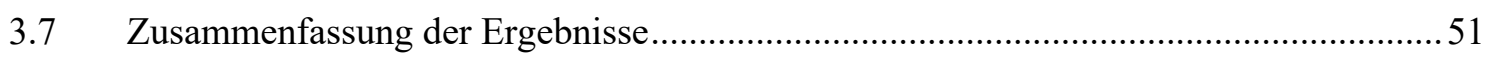

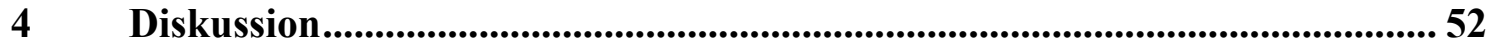

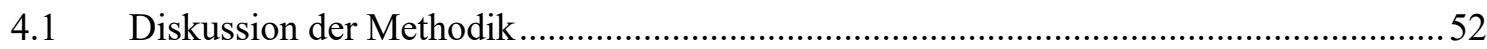

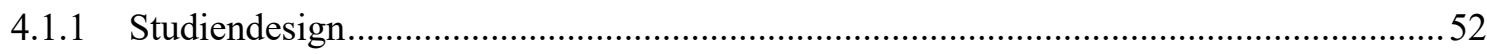

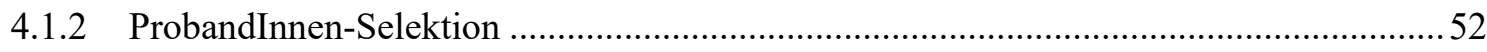

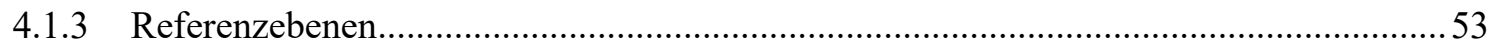

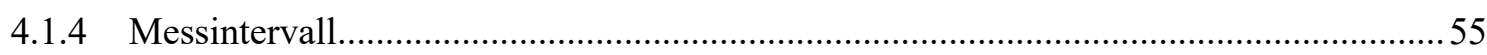

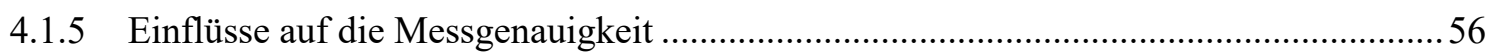

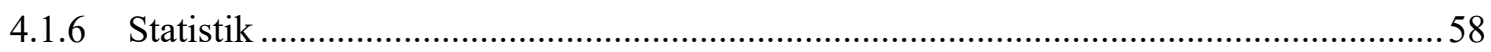

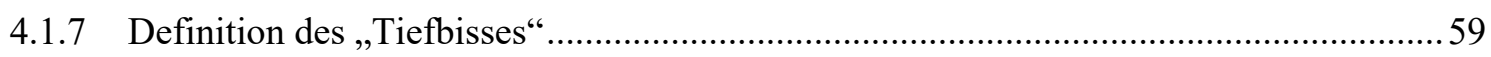

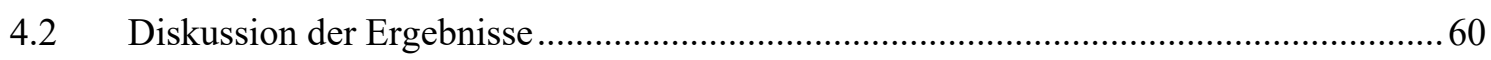

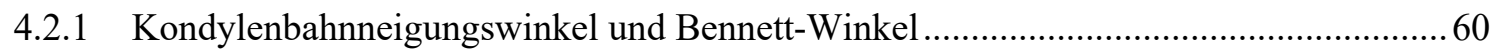

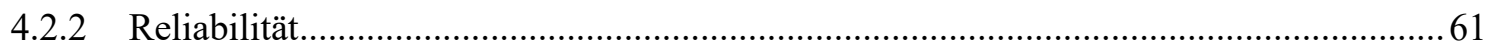

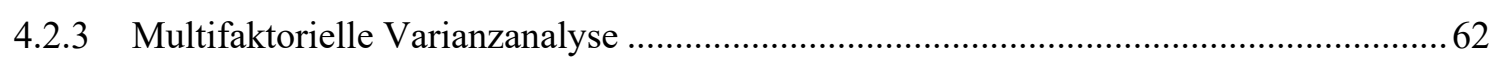

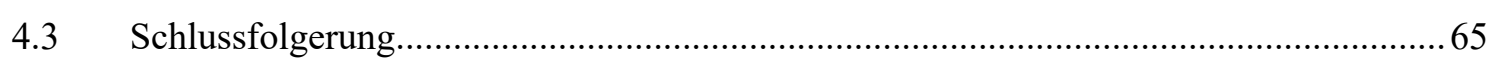

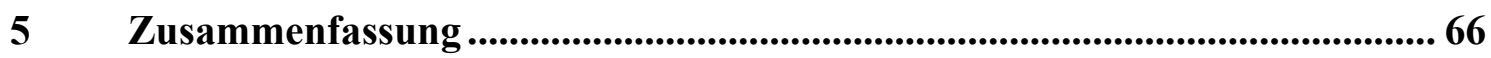

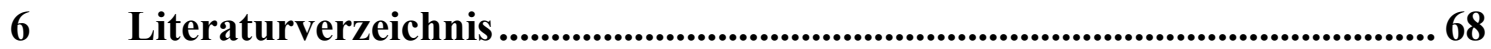




\section{Abbildungsverzeichnis}

Abbildung 1: Schematische Darstellung des Kiefergelenks in der Sagittalsicht.......................... 3

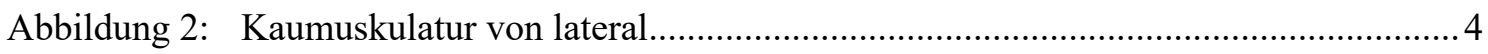

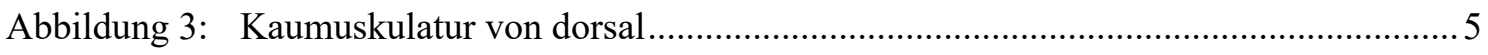

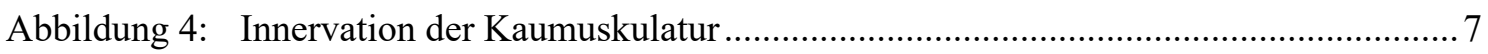

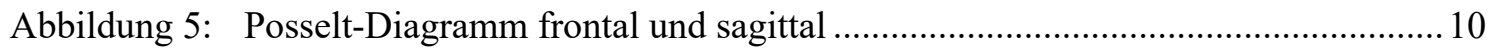

Abbildung 6: Kondylenbahnwinkel mit der Frankfurter Horizontalen als Referenzebene ....... 12

Abbildung 7: Versatz der Kondylen bei Laterosionsbewegung .............................................. 14

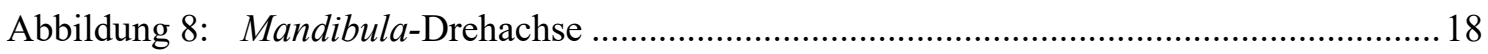

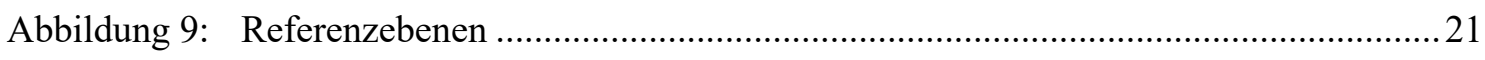

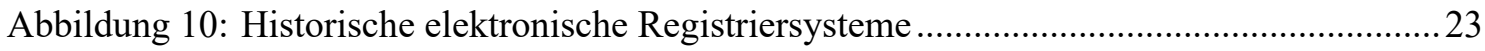

Abbildung 11: Datenerfassung und Darstellung auf dem ARCUSdigma-II-Monitor..................25

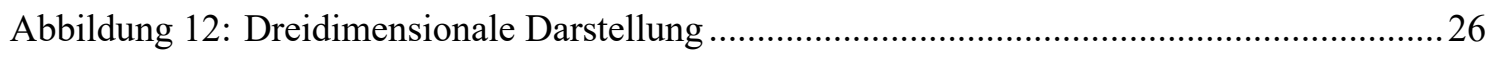

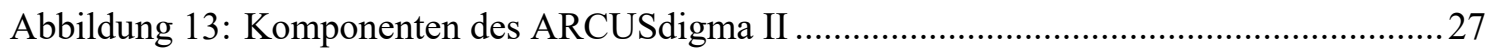

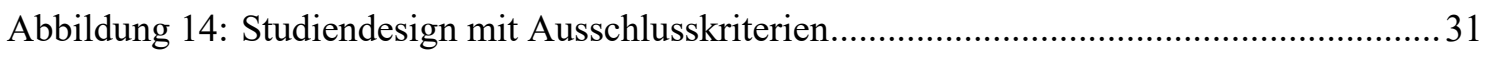

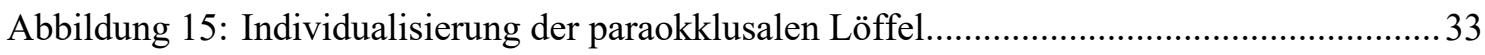

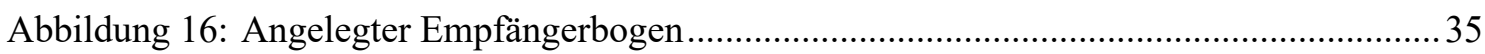

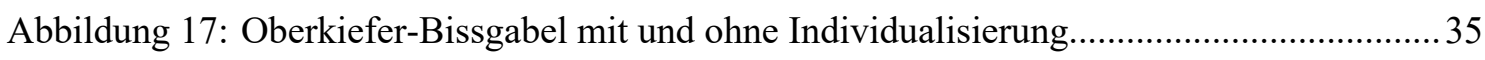

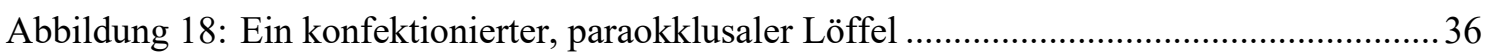

Abbildung 19: Einsetzen des Basisplattenwachses zum Vermeiden von Störkontakten ............. 37

Abbildung 20: Adaption des paraokklusalen Löffels an die Unterkieferzähne............................. 38

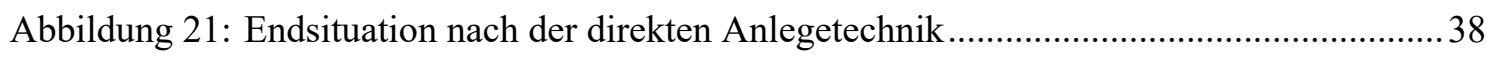

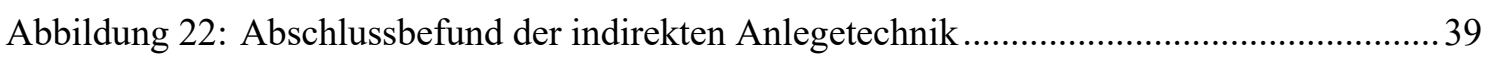

Abbildung 23: Während der Messung durchgeführte Unterkieferbewegungen .......................... 41

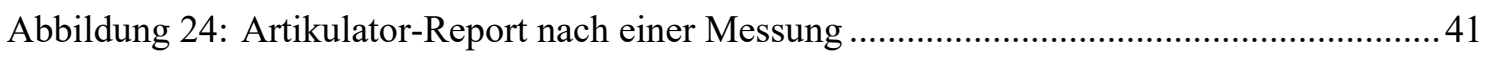

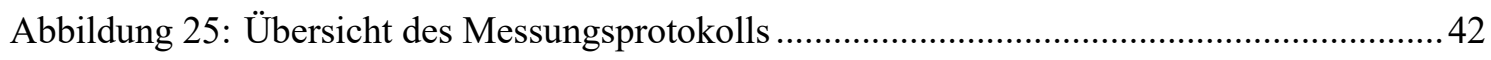

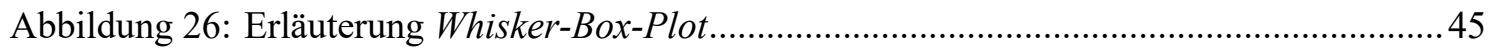

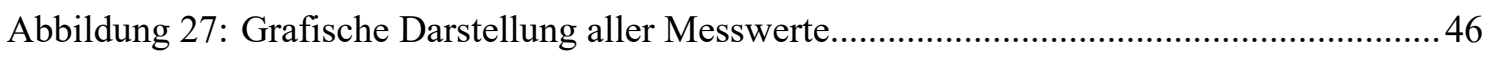

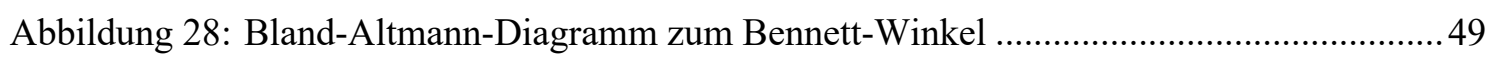

Abbildung 29: Bland-Altmann-Diagramm zum Kondylenbahnneigungswinkel .........................49

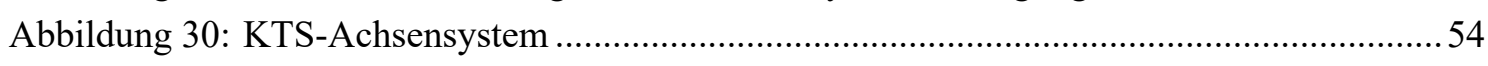

Abbildung 31: Tiefbiss und die daraus resultierende Reduktion der Retentionsfläche................59

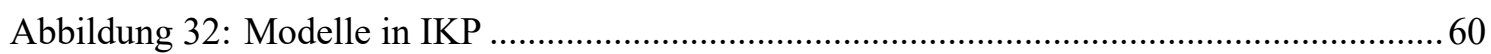




\section{Tabellenverzeichnis}

Tabelle 1: $\quad$ Literaturübersicht über die sagittale Kondylenbahnneigung................................ 11

Tabelle 2: $\quad$ Literaturübersicht über Untersuchungsergebnisse zur Messung des Bennett-

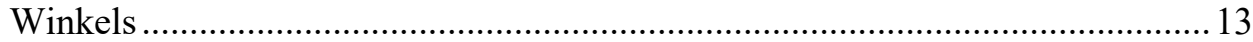

Tabelle 3: $\quad$ BA-Messwerte mit der größten Inter-Session-Differenz zur Überprüfung der Test-Retest-Reliabilität der direkten Messtechnik .47

Tabelle 4: $\quad$ BA-Messwerte mit der größten Inter-Session-Differenz zur Überprüfung der Test-Retest-Reliabilität der indirekten Messtechnik.................47

Tabelle 5: $\quad$ HCI-Messwerte mit der größten Inter-Session-Differenz zur Überprüfung der Test-Retest-Reliabilität der direkten Messtechnik

Tabelle 6: $\quad$ HCI-Messwerte mit der größten Inter-Session-Differenz zur Überprüfung der Test-Retest-Reliabilität der indirekten Messtechnik. .48

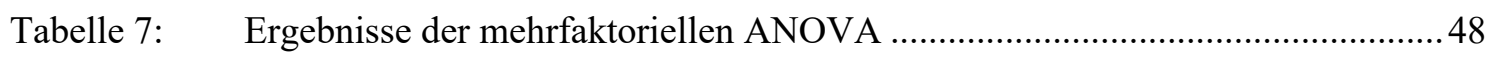

Tabelle 8: $\quad$ Vergleich direkte Anlegetechnik und indirekte Anlegetechnik ...........................50 


\section{Abkürzungsverzeichnis}

BA

$\mathrm{CAD} / \mathrm{CAM}$

CMD

EAEF

EMG

EPA

HCI

IKP

ISS

KTS

Lig.

Ligg.

M.

$\mathrm{Mm}$.

MRT

N.

Nn.
Bennett-Winkel

computer-aided design/computer-aided manufacturing

Craniomandibuläre Dysfunktion

elektronische Analyse ätiologischer Faktoren

Elektromyographie

electronic position analysis

Kondylenbahnneigungswinkel

maximale Interkuspidation

immediate side shift

KaVo-Transfer-System

lateinisch: Ligamentum

lateinisch: Ligamenti

lateinisch: Musculus

lateinisch: Musculi

Magnetresonanztomographie

lateinisch: Nervus

lateinisch: Nervi 


\section{$1 \quad$ Einleitung}

In den letzten Jahren ist die Diagnostik und Therapie funktioneller Störungen des stomatognathen Systems immer mehr in den Fokus der klinischen und wissenschaftlichen Zahnmedizin gerückt. Dabei wurden die manuellen und digitalen Methoden der klinischen Funktionsdiagnostik immer weiterentwickelt, um die traditionellen, ausschließlich auf Bewegungsanalysen des Kondylus fokussierten Techniken zu ersetzen. So werden aktuell zur Analyse der Unterkieferfunktion nicht mehr nur die zweidimensionalen Bewegungsbahnen einzelner Fixpunkte registriert, sondern die dreidimensionalen Bewegungen des gesamten Unterkiefers in Bezug auf alle beteiligten anatomischen Strukturen. Die Bewegungen des Unterkiefers werden also in allen Dimensionen, inklusive der Zeit, erfasst und ausgewertet, sodass eine objektive Bewertung und Beurteilung von eventuellen Pathologien, z. B. Craniomandibulären Dysfunktionen (CMD), unterstützt und präzisiert wird. Neue digitale Messtechniken sind zunehmend in der Lage, die Unterkieferbewegungen exakter und weniger invasiv zu erfassen bzw. abzubilden und liefern somit objektivere und reproduzierbare Werte für diagnostische und therapeutische Prozedere.

Die Erhebung funktionsanalytischer Daten wird heute zur Unterstützung in der klinischen Diagnostik von CMD oder vor prothetischen Rehabilitationen des Kausystems zur Verbesserung der Therapieerfolge eingesetzt. Volljustierbare Artikulatoren werden zur extraoralen Simulation von Unterkieferbewegungen beispielweise mittels BennettWinkel, Kondylenbahnneigungswinkel, interkondylärer Distanz und räumlicher Zuordnung von Oberkiefer zu Unterkiefer und der vermeintlichen Scharnierachse mit Hilfe eines Gesichtsbogens programmiert. In der aktuellen Literatur wird die umfassende Erhebung dieser Daten und die Verwendung eines Gesichtsbogens zur Herstellung von Zahnersatz kontrovers diskutiert. Die individuelle Erhebung von Bennett-Winkel und Kondylenbahnneigungswinkel führt jedoch nachweislich zur Reduktion erforderlicher Einschleifmaßnahmen und damit zur Steigerung der Qualität und der Überlebensraten bei umfangreichen prothetischen Versorgungen. Aktuell verfügbare elektronische Messsysteme unterstützen die digitale Erhebung dieser Daten und sind auch bei niedergelassenen Behandlern relativ weit verbreitet. 
Die Messungen bei ultraschallbasierten und optischen Systemen erfolgt berührungslos, wobei beide Verfahren die Anwendung von okklusionsbedeckenden (periokklusalen) oder paraokklusalen Löffeln zum Fixieren des Gerätes an der Unterkieferdentition benötigen. Diese Löffel können direkt am Patienten (chairside) oder indirekt (vorher im Dentallabor) hergestellt werden. Die Validität und insbesondere Reliabilität solcher moderneren Techniken sind bisher jedoch nicht ausreichend untersucht worden.

In dieser Studie sollte die Reliabilität solcher elektronischer Registriersysteme am Beispiel des ARCUSdigma II der Firma KaVo (Fa. KaVo) bei der Artikulatorprogrammierung untersucht werden, um dann eventuell eine Evidenz-basierte klinische Empfehlung geben $\mathrm{zu}$ können, in welchen Situationen das jeweilige Anlegeverfahren durchzuführen ist. Zusätzlich sollte die Praktikabilität beider Anlegeverfahren in Abhängigkeit zur Ausprägung des frontalen Überbisses evaluiert werden, um verlässliche Empfehlungen für den klinischen Alltag im Umgang mit einem elektronischen Axiographen zu formulieren.

\subsection{Literaturübersicht}

\subsubsection{Die Anatomie des Kiefergelenks}

Als einziges Doppelgelenk des menschlichen Körpers ist das Kiefergelenk aufgrund der anatomischen und biomechanischen Gegebenheiten hochkomplex (Kober et al. 2016). Die knöchernen Strukturen der Gelenkpfanne werden von Anteilen des Schläfenbeins (Tuberculum articulare und Fossa mandibularis) und des Unterkiefers (Kondylus mandibulae) gebildet (siehe Abbildung 1). Die Fossa mandibularis wird anterior durch das Tuberculum articulare und dorsal von dem Processus postglenoidalis begrenzt und stellt mit ihrer konkaven Form eine Vertiefung für den Kondylus mandibulae dar (Öberg et al. 1971). Unter physiologischen Bedingungen sind diese knöchernen Gelenkkörper von Faserknorpel unterschiedlicher Schichtdicke überzogen. Der bindegewebige Discus articularis lässt sich in eine anteriore und mediale sowie eine posteriore Zone unterteilen. Der posteriore Anteil bildet die dorsale Anheftung des Discus und ist aus einer superioren und einer inferioren Lamina aufgebaut, er wird aufgrund dieser doppelten Laminae auch bilaminäre Zone genannt (siehe Abbildung 1). Der anteriore Teil des Discus ist mit der Gelenkkapsel und dem Caput infratemporale des Musculus Pterygoideus lateralis (M.) mit Kollagenfasern verbunden und besteht aus fribrösem Anteil mit eingestreuten Knorpelzellen. Der mediale Teil ist von Faserknorpel geprägt und ist im Vergleich zur 
posterioren, bilaminären Zone nicht so stark vaskularisiert und innerviert (Okeson und de Leeuw 2011). Diese sensible Innervation macht es möglich, dass schon kleine Veränderungen als Schmerz oder Druck wahrgenommen werden können (Ahlers und Jakstat 2008). Der superiore Anteil der bilaminären Zone ist am hinteren Rand der Fossa mandibularis verankert und besteht hauptsächlich aus fibroelastischem Gewebe, während der inferiore Anteil aus sehr fibrösem und straffem Gewebe gebildet und am Hinterrand des Kondylus mandibulae befestigt ist. Die Gelenkkapsel weist mehrere Reservefalten auf und besteht aus einer dünnen, an elastischen Fasern reichen, bindegewebigen Schicht. Das lateral eingebettete Ligamentum laterale (Lig.) sowie die Ligamenti stylomanibulare und sphenomandibulare (Ligg.) tragen zur Verstärkung der Kapsel bei und sichern den physiologischen Bewegungsspielraum des Gelenks (Strub et al. 2011).

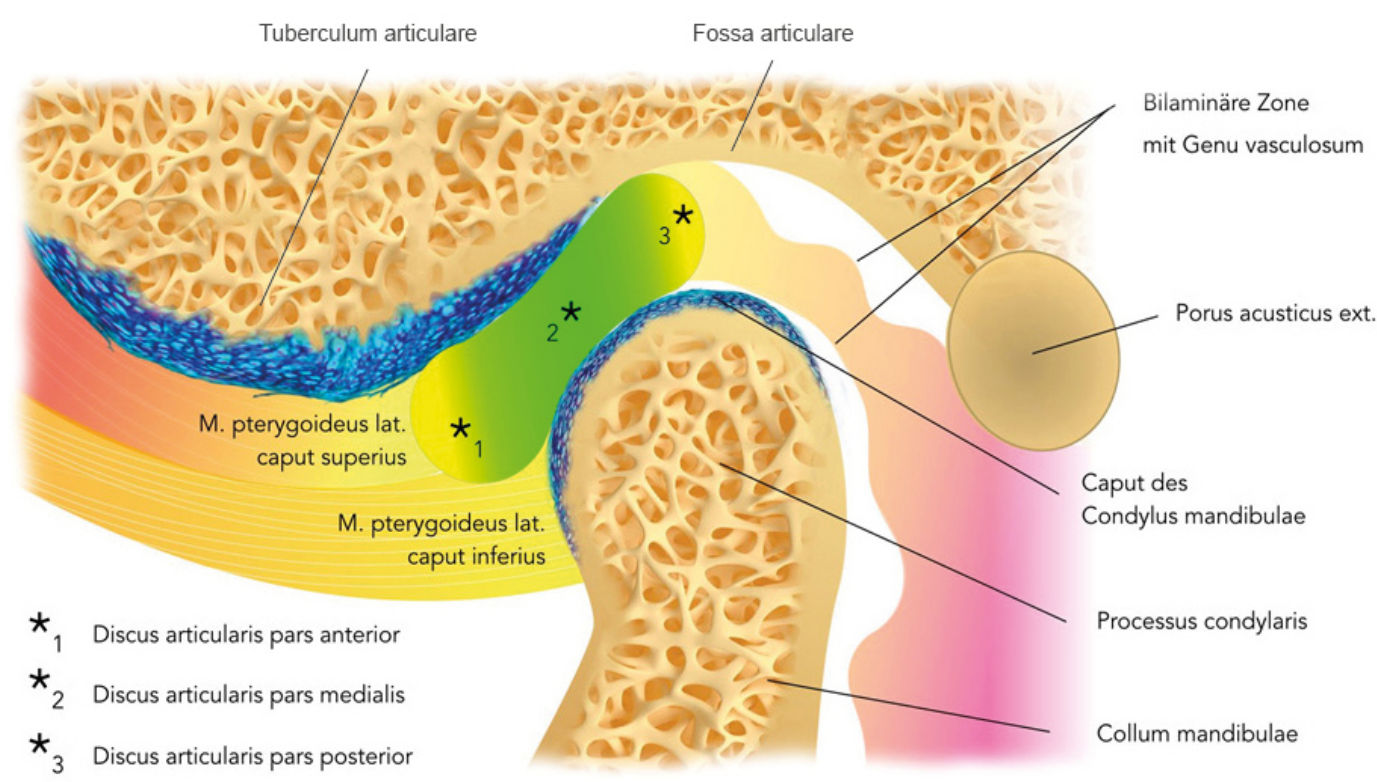

Abbildung 1: Schematische Darstellung des Kiefergelenks in der Sagittalsicht (Quelle: S. Krohn, Göttingen)

\subsubsection{Die Kaumuskulatur}

Die Kaumuskeln bilden mit den Kiefergelenken und mit den Zähnen eine funktionelle Einheit. Die vier Muskelpaare Musculi temporalis (Mm.), Mm. masseter, Mm. pterygoidei lateralis und Mm.pterygoidei medialis (siehe Abbildung 2 und Abbildung 3), die motorisch von Ästen des Nervus mandibularis (N.) des fünften Hirnnervs ( $N$. trigeminus) innerviert werden, nehmen einen direkten Einfluss auf die Kieferbewegungen und werden hier deshalb genauer beschrieben (siehe Abbildung 4) (Moll und Moll 2006). Die Muskulatur des Mundbodens, die infra- und suprahyalen 
Muskeln sowie die Halsmuskulatur unterstützen die Kaumuskeln in ihrer Funktion, den Unterkiefer gegen den Schädel zu bewegen (Lippert und Deller 2004).

\subsubsection{M. temporalis}

Der M. temporalis besteht aus zwei Anteilen und entspringt fächerförmig an der Fossa temporalis sowie von der Innenseite der Fascia temporalis, die ihn von außen komplett umgibt. Die Fasern ziehen medial am Os zygomaticum vorbei und bilden mit einer kräftigen Sehne den Ansatz am Processus coronoideus und am vorderen aufsteigenden Unterkieferast (Sobotta et al. 2005). Der M. temporalis ist einzeln betrachtet der größte Kaumuskel und weist durch seine unterschiedlich verlaufenden Fasern mehrere Funktionen auf (siehe Abbildung 2 und Abbildung 3) (Hugger et al. 2006). Während die mittleren und hinteren Fasern für Retrusionsbewegungen und Mundschluss verantwortlich sind, dienen die nahezu vertikal verlaufenden vorderen Fasern dazu, den Unterkiefer zu protrudieren. Für die Innervation sind die Nervi temporalis profundi (Nn.) aus dem dritten Ast (N. mandibularis) des N. trigeminus zuständig (siehe Abbildung 4) (Schumacher 1984).

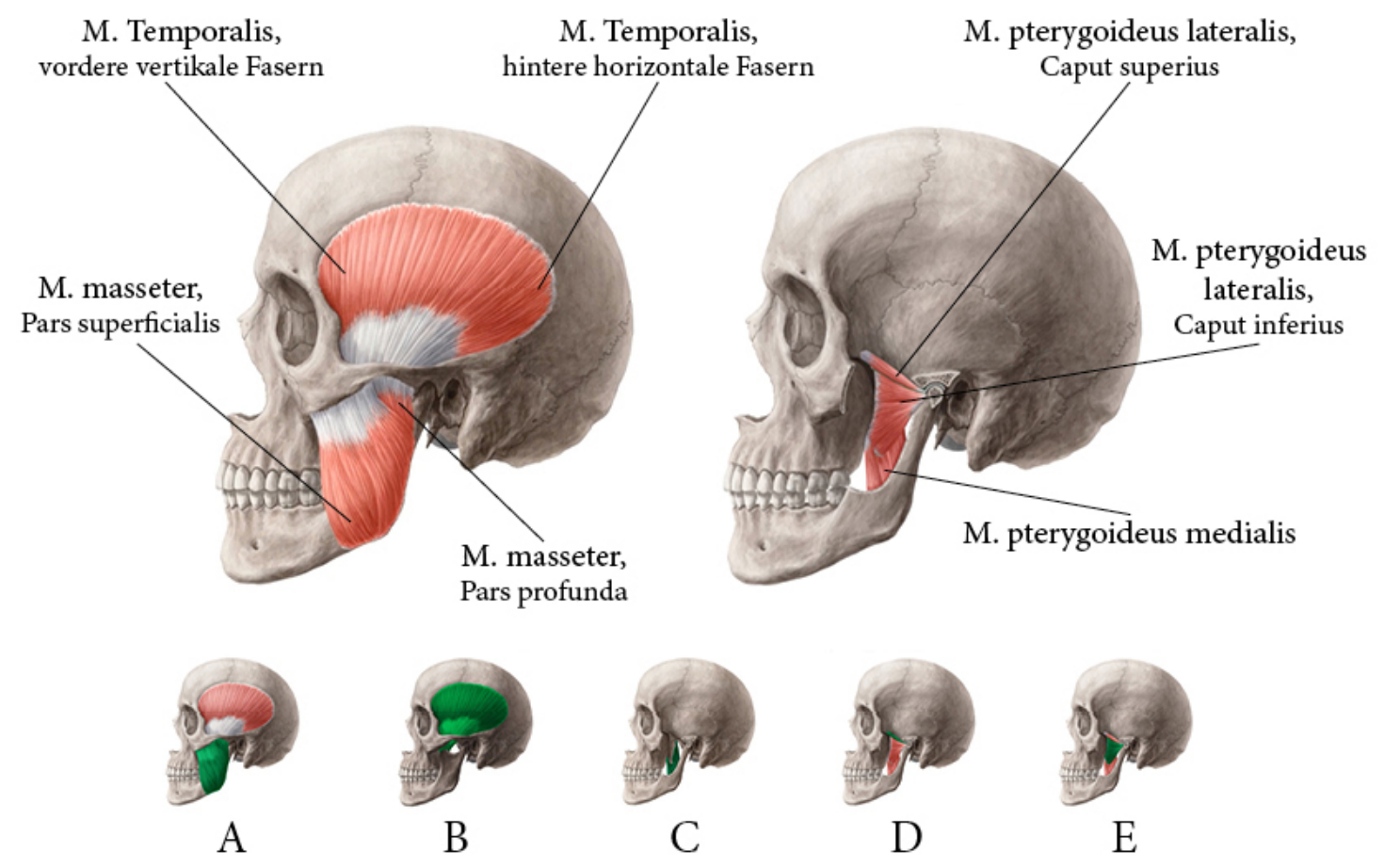

Abbildung 2: Kaumuskulatur von lateral:

$\mathrm{A}=$ M. masseter,$\quad \mathrm{B}=$ M. temporalis,$\quad \mathrm{C}=$ M. pterygoideus medialis, $\mathrm{D}=$ M. pterygoideus lateralis (Caput superius), $\mathrm{E}=$ M. pterygoideus lateralis (Caput inferius) Modifiziert nach Yousun Koh (Koh 2020b) mit freundlicher Genehmigung von Kennhub 


\subsubsection{M. masseter}

Der kräftige $M$. masseter lässt sich hinsichtlich seines unterschiedlichen Faserverlaufs in einen oberflächlichen Teil und einen tiefen Anteil unterscheiden (siehe Abbildung 2). Beide Anteile nehmen ihren Ursprung am Arcus zygomaticus. Der tiefe Teil des M. masseter (pars profunda) mit dem Ansatz an der Außenseite des Ramus mandibulae des Unterkiefers sowie der oberflächliche Teil (pars superficialis), der außen am Kieferwinkel an der Tuberositas masseterica ansetzt, sind aufgrund ihrer unterschiedlichen Verläufe bei der Protrusions-, Retrusions- und Laterotrusionsbewegung des Unterkiefers beteiligt (siehe Abbildung 2 und Abbildung 3) (Sobotta et al. 2005). Die Adduktion ist jedoch die Hauptfunktion des M. masseter. Seine Kontraktion führt gemeinsam mit den anderen Kaumuskeln zu einem kräftigen Kieferschluss und ist zusätzlich an der Aufrechterhaltung der Spannung der Kiefergelenkkapsel beteiligt. Wie der M. temporalis wird auch der M. masseter durch einen Ast des N. mandibularis innerviert, den N. massetericus (siehe Abbildung 4) (Bumann und Lotzmann 2000).

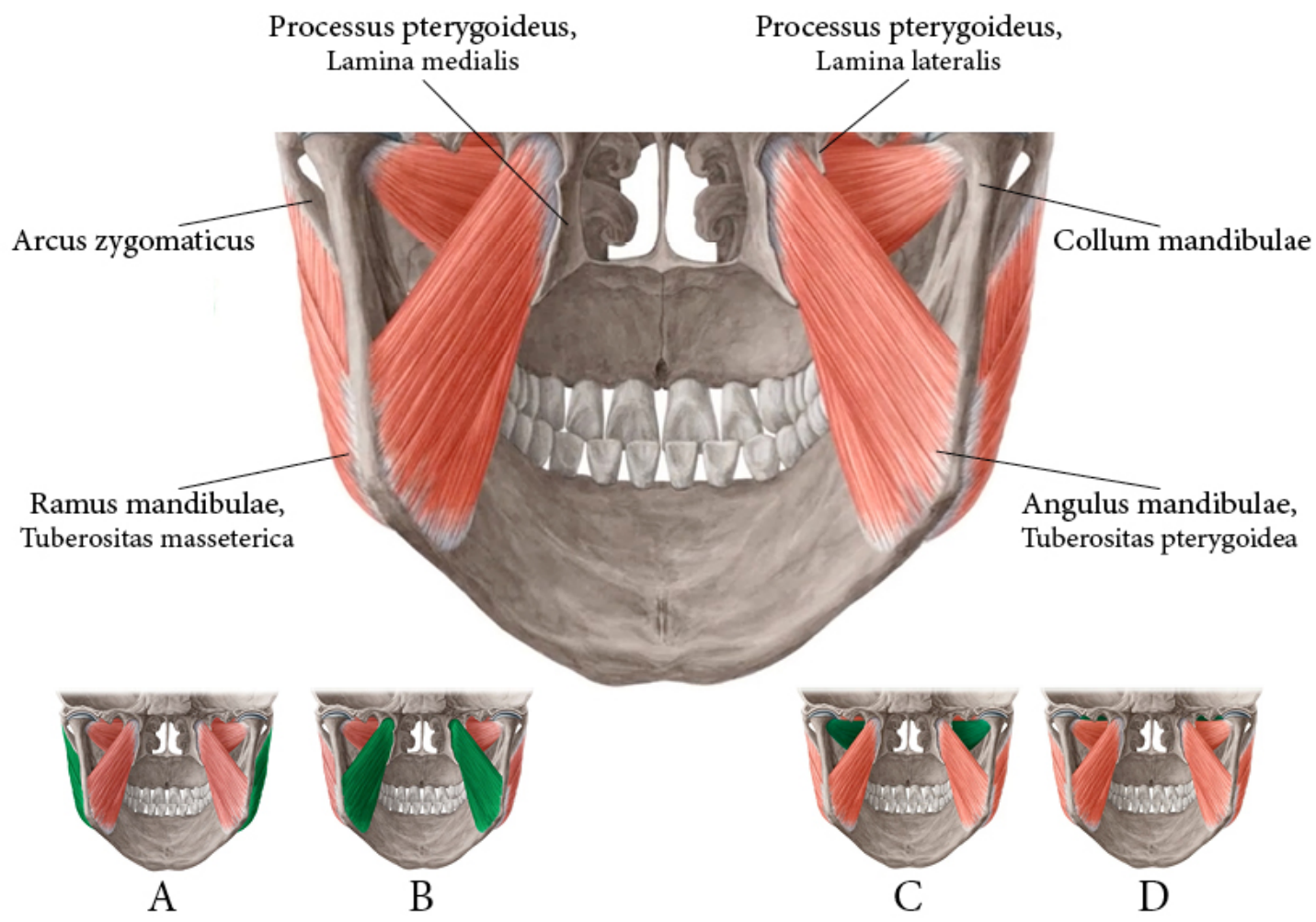

Abbildung 3: Kaumuskulatur von dorsal:

$\begin{array}{llll}\mathrm{A}= & \text { M. masseter, } & \mathrm{B}= & \text { M. pterygoideus medialis, } \\ \mathrm{C}= & M . \text { pterygoideus lateralis } & \text { (Caput inferius), } \\ \mathrm{D}= & \text { M. pterygoideus lateralis } & \text { (Caput superius) }\end{array}$

Modifiziert nach Yousun Koh (Koh 2020a) mit freundlicher Genehmigung von Kennhub 


\subsubsection{M. pterygoideus medialis}

An der medialen Seite des Unterkiefers liegt der M. pterygoideus medialis und entspringt an der Lamina medialis des Processus pterygoideus und an der Fossa pterygoidea des Os sphenoidale (siehe Abbildung 2 und Abbildung 3) (Sobotta et al. 2005). Durch seinen Ansatz an der Tuberositas pterygoidea des Angulus mandibulae bildet er mit dem M. masseter eine funktionelle Muskelschlinge, die durch die Kontraktion beider Muskeln einen kräftigen Zug am Unterkiefer nach kranial bewirkt (Waldeyer und Mayet 1993). Außerdem ist der M. pterygoideus medialis entsprechend seines Verlaufs an der Laterotrusion, Protrusion und Retrusion des Unterkiefers beteiligt (Hugger et al. 2006). Über den N. pterygoideus medialis aus dem N. mandibularis erfolgt seine Innervation (siehe Abbildung 4) (Bumann und Lotzmann 2000).

\subsubsection{M. pterygoideus lateralis}

Der M. pterygoideus lateralis ist im Vergleich $\mathrm{zu}$ den anderen bereits erwähnten Kaumuskeln der kürzeste Muskel und verläuft mit seiner kegeligen Form nahezu horizontal zwischen dem Kondylus des Unterkiefers und der Fossa infratemporalis (siehe Abbildung 2 und Abbildung 3). Er hat einen kleineren, oberen Kopf (Caput superius) und einen weitaus größeren, unteren Kopf (Caput inferius), die unterschiedlich funktionell wirken (Sobotta et al. 2005). Der kleinere, obere Kopf entspringt an der Ala major ossis sphenoidalis sowie der Crista infratemporalis ossis sphenoidalis und setzt sowohl am oberen Anteil der Fovea pterygoidea als auch am medialen Drittel des Discusbandes anterior an. Der Ursprung des unteren Kopfes ist die Außenseite der Lamina lateralis des Processus pterygoidei und die Insertion ist am Collum mandibulae in der Fovea pterygoidea. Einige Muskelfasern dieses größeren Kopfes strahlen in den Discus ein (Griffin et al. 1975; Dauber 1987; Abe et al. 1993). Eine histologische Untersuchung von Sümnig et al. (1991) ergab, dass der Muskel in den Discus articularis und in die Kapsel einstrahlt. 


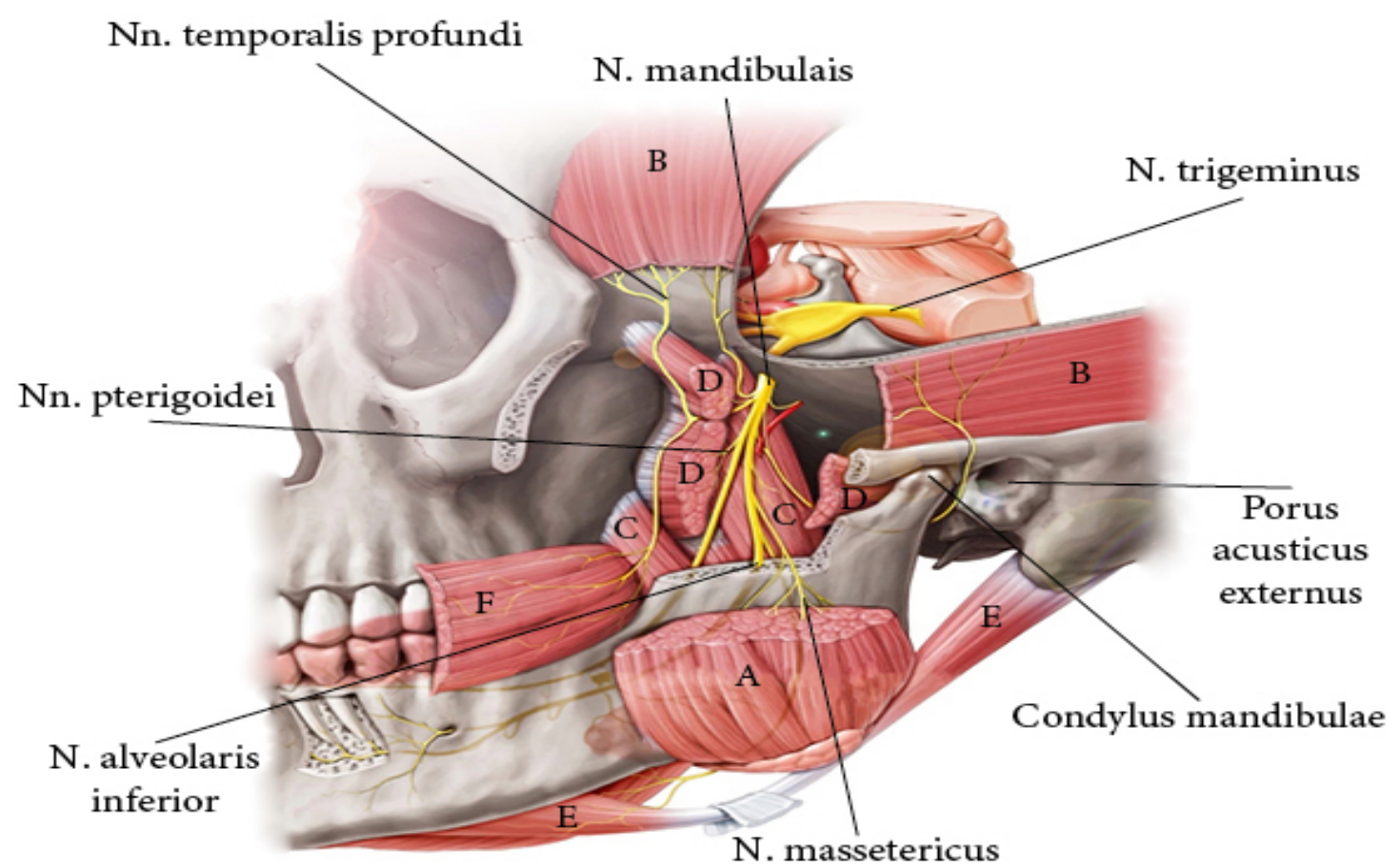

Abbildung 4: Innervation der Kaumuskulatur:

$\mathrm{A}=$ M. masseter,$\quad \mathrm{B}=$ M. temporalis (vordere $\mathrm{u}$. hintere Anteile), $\mathrm{C}=M$. pterygoideus medialis,$\quad \mathrm{D}=M$. pterygoideus lateralis, $\mathrm{E}=$ M. digastricus (Venter anterior $\mathrm{u}$. posterior), $\quad \mathrm{F}=$ M. buccinator Modifiziert nach Paul Kim (Kim 2020) mit freundlicher Genehmigung von Kennhub

\subsubsection{Mobilität des Unterkiefers}

\subsubsection{Mundöffnung und Mundschließung}

Die Mundöffnung besteht aus einer kombinierten Rotations- und Translationsbewegung. Nach Tonusverminderung der Schließmuskulatur erfolgt die intitiale Mundöffnung zunächst durch die Schwerkraft (Lippert und Deller 2004). Da eine reine Rotationsbewegung aufgrund der engen Platzverhältnisse in der Fossa retromandibularis nicht möglich ist, kontrahiert bei der Mundöffnung der M. pterygoideus lateralis und ermöglicht so das Gleiten des Kondylus-Discus-Komplexes entlang der Fossa mandibularis nach vorne unten auf das Tuberculum articulare (Hugger et al. 2006). Die maximal mögliche Mundöffnung erfolgt anschließend durch das aktive Ziehen der suprahyalen Muskulatur an der Mandibula nach unten, während die infrahyalen Muskeln das Zungenbein fixieren und das Ligamentum laterale die Gelenke stabilisiert (Gallo et al. 1997). Diese Kombination aus Rotations- und Translationsbewegungen variiert in ihren Anteilen in Abhängigkeit von der Gelenkanatomie (Ettlin und Gallo 2018). Der Discus articularis und der Kondylus werden in der Regel zeitgleich nach ventral bewegt, 
wobei der Hauptteil der Kondylenrotation im Bereich der medialen Zone des Discus articularis stattfindet. Durch die Bewegung des Discus wird der superiore Anteil der bilaminären Zone zum Teil ins Gelenk gezogen, wodurch der venöse Plexus sich mit Blut füllt. Der inferiore Anteil der bilaminären Zone ist dabei für die Stabilisierung des Discus verantwortlich (Siebert 1996).

Für die Mundschließung kontrahieren die Mm. temporalis, Mm. masseter und Mm. pterygoidei medialis und schaffen gemeinsam die Rückverlagerung der Gelenkkomponenten (Sobotta et al. 2005). Der Vorgang, welcher zur Rückführung des Discus articularis in die Fossa führt, ist nicht abschließend geklärt. Dieser könnte durch die elastischen retrodiskalen Fasern erfolgen oder der Discus folgt der Kondylenbewegung und repositioniert sich passiv (Wilkinson und Crowley 1994).

\subsubsection{Protrusion, Retrusion und Laterotrusion}

Bei der Protrusion wird der Unterkiefer nach anterior bewegt, nach posterior wird die Bewegung als Retrusion und zur Seite als Laterotrusion bezeichnet (Hugger et al. 2006). Durch die kombinierte symmetrische Kontraktion des M. pterygoideus medialis, des M. pterygoideus lateralis und Anteilen des M. masseter kann die Protrusion des Unterkiefers durchgeführt werden (Koeck et al. 1995). Dabei wird der Kondylus-DiscusKomplex aus der Fossa articularis nach ventrokaudal entlang des Tuberculum articulare verschoben (Siebert 1996). Bei der Retrusion werden die Gelenkköpfe hauptsächlich durch die Kontraktion der hinteren Fasern des M. temporalis zurück verlagert, wobei die tiefen Anteile des M. masseter sowie die M. genihyoideus und der M. digastricus diese Bewegung unterstützen (Ptok 2014). Die Laterotrusionsbewegungen werden hauptsächlich durch den M. pterygoideus lateralis und M. pterygoideus medialis koordiniert (Schumacher 1984). Hier kommt es zu einer einseitigen Verlagerung des Kondylus (Mediotrusionskondylus) nach ventromedial, während auf der Gegenseite in der Gelenkfossa eine nach ventrolaterale Verschiebung durch die Rotation des Kondylus (Laterotrusionskondylus) stattfindet (Palla et al. 2003). Durch die einseitige Kontraktion des M. pterygoideus lateralis sowie des M. masseter und M pterygoideus medialis wird der Mediotrusionskondylus auf das Tuberculum articulare verlagert, während die gleichzeitige Kontraktion des M. temporalis die Rotation des Laterotrusionskondylus bewirkt (Koeck et al. 1995). Die Führung der Protrusions- und Laterotrusionsbewegungen werden zudem maßgeblich durch die Stellung der Zahnreihen beeinflusst (Pröschel 1988). 


\subsubsection{Die Bewegungen des Unterkiefers}

Bei jeder Bewegung des Unterkiefers bilden die Zahnreihen, die beiden Kiefergelenke und das neuromuskuläre System - als die drei Hauptkomponenten des Kauapparats - eine funktionelle Kette und beeinflussen sich gegenseitig (Siebert 1996). Dabei muss man zwischen den funktionellen Kaubewegungen und den Grenzbewegungen des Kauapparates differenzieren (Sander et al. 2011). Als Grenzbewegungen werden die maximalen Exkursionsbewegungen des Unterkiefers bezeichnet, die nur selten habituell durchgeführt werden (Strub et al. 2011). Die häufigste, gewohnheitsmäßig eingenommene Unterkieferposition ist die entspannte Ruhelage ohne Zahnkontakt (Ahlers et al. 2006). In dieser sogenannten Ruhelage (früher: Ruheschwebelage) befindet sich der Tonus der Muskulatur (Unterkieferheber und -senker) im Gleichgewicht (Wenz und Hellwig 2018). Die Kondylenposition bei dieser Lage ist unbestimmt, da sie sehr stark vom variablen Muskeltonus abhängig ist (Michelotti et al. 1997). Aufgrund dieser Impräzision werden die meisten Untersuchungen der Unterkieferbewegungen aus der Position der maximalen Interkuspidation (IKP) gestartet (Ettlin und Gallo 2018). Die maximale IKP wird bei Menschen, die eine stabile Verzahnung mit weitgehend erhaltenen Stützzonen haben, durch die Okklusion determiniert (Ahlers et al. 2006). Hier wird die dreidimensionale Relation des Unterkiefers zum Oberkiefer durch die Okklusion definiert und somit die Stellung der Kondylenposition in den Fossae articularis bestimmt (The Glossary of Prosthodontic Terms: Ninth Edition 2017). Durch verschiedene instrumentelle Analysen lassen sich alle Grenzbewegungen in der sagittalen, vertikalen und horizontalen Ebene erfassen (Sander et al. 2011). Für die Darstellung der Grenzbewegungen des Unterkiefer-Inzisalpunktes in der Horizontalebene eignet sich der sogenannte gotische Bogen und in der Sagittal- und Transversalebene das PosseltDiagramm (siehe Abbildung 5) (Posselt 1958). 


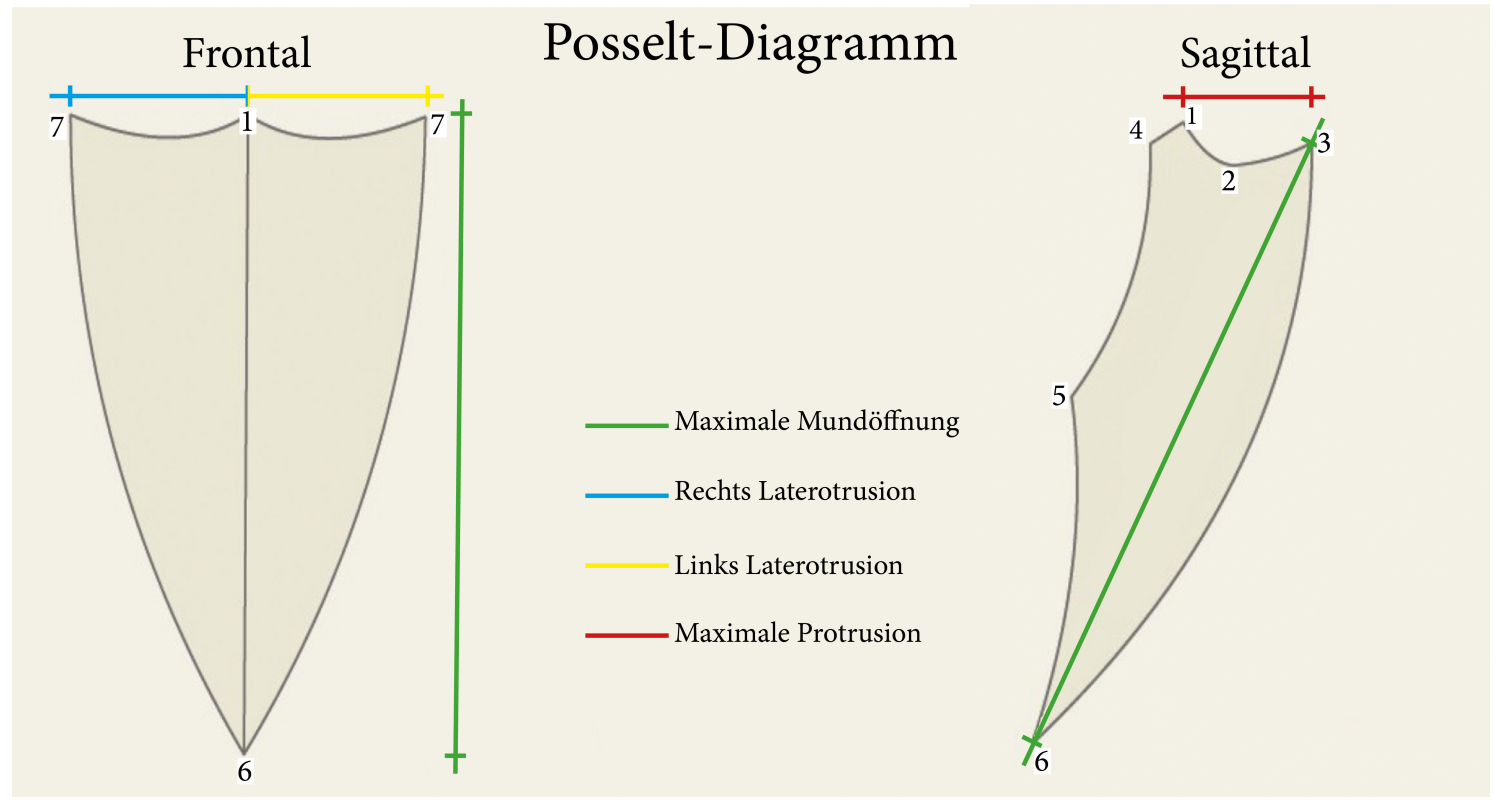

Abbildung 5: Posselt-Diagramm frontal und sagittal:

1 = Maximale Interkuspidation, $\quad 2$ = Kopfbissstellung, $3=$ Maximale Protrusion, $\quad 4$ = Retrudierte Kontaktposition,$\quad 5=$ Mundöffnung durch reine Rotationsbewegung, 6 = Maximale Mundöffnung, $7=$ Maximale Laterotrusion

\subsubsection{Kondylenbahnneigungswinkel}

Die Grenzbewegungen des Kondylus können ebenso in der sagittalen, transversalen und horizontalen Ebene betrachtet und beschrieben werden (Freesmeyer et al. 1992). Während der Protrusion führt der Kondylus eine Bewegung nach ventrokaudal durch (Ptok 2014). Bedingt durch Gleiten des Kondylus auf das Tuberculum articulare zeigt die sagittale Betrachtung dieser Bewegung keine gerade, sondern eine bogenförmige Kondylenbahn (siehe Abbildung 6) (Benner et al. 1993). Das Ziehen einer Gerade zwischen dem Ausgangspunkt des Kondylus (maximale Interkuspidation) und ihrer Positionen auf der Kondylenbahn nach $5 \mathrm{~mm}$ Protrusion bildet einen Winkel zu einer Referenzebene am Schädel (siehe Kapitel 1.1.5.3 Bezugsebenen) (Gonzalez und Kingery 1968; Bumann und Lotzmann 2000). Dieser Winkel ist der Kondylenbahnneigungswinkel (HCI) und ist für die Programmierung von Artikulatoren eine entscheidende Größe (Downs 1948; Körber und Luckenbach 1981). Da die Frankfurter Horizontale als Referenzebene im Raum zur Kondylenbahn steiler ist als die CamperEbene (siehe Kapitel 1.1.5.3 Bezugsebenen), sind die Werte der Kondylenbahnneigungswinkel mit dieser um durchschnittlich $15^{\circ}$ größer (Wenz und Hellwig 2018). Eine Übersicht der durchschnittlichen sagittalen Kondylenbahnneigungswinkel aus der Literatur mit den jeweiligen Bezugsebenen ist in Tabelle 1 aufgeführt. 
Tabelle 1: $\quad$ Literaturübersicht über die sagittale Kondylenbahnneigung

\begin{tabular}{|c|c|c|c|c|}
\hline Autor und Jahr & $\begin{array}{c}\text { Anzahl der } \\
\text { ProbandInnen }\end{array}$ & $\begin{array}{c}\text { Alter der } \\
\text { ProbandInnen }\end{array}$ & $\begin{array}{c}\text { Mittlere } \\
\text { SKN }\end{array}$ & Bezugsebene \\
\hline $\begin{array}{l}\text { Gysi } \\
(1908)\end{array}$ & 34 & unbekannt & $33,00^{\circ}$ & $\mathrm{CE}$ \\
\hline $\begin{array}{l}\text { Isascson } \\
(1959)\end{array}$ & 36 & 43,6 (mean) & $35,60^{\circ}$ & $\mathrm{FH}$ \\
\hline $\begin{array}{l}\text { Posselt } \\
(1960)\end{array}$ & 10 & unbekannt & $40,00^{\circ}$ & $\mathrm{CE}$ \\
\hline $\begin{array}{l}\text { Lundeen et al. } \\
\text { (1973) }\end{array}$ & 50 & $20-55$ & $40,00^{\circ}$ & FH \\
\hline $\begin{array}{l}\text { Kohno et al. } \\
\text { (1987) }\end{array}$ & 35 & 25,8 (mean) & $38,20^{\circ}$ & $\mathrm{CE}$ \\
\hline $\begin{array}{l}\text { Zamacona et al. } \\
\text { (1992) }\end{array}$ & 56 & 63,3 (mean) & $36,20^{\circ}$ & $\mathrm{CE}$ \\
\hline $\begin{array}{l}\text { Johnson/Winstanley } \\
\text { (1997) }\end{array}$ & 103 & $18-32$ & $31,75^{\circ}$ & $\mathrm{CE}$ \\
\hline $\begin{array}{l}\text { Hernandez et al. } \\
\text { (2010) }\end{array}$ & 45 & 31,4 (mean) & $48,80^{\circ}$ & FH \\
\hline $\begin{array}{l}\text { Schierz et al. } \\
\text { (2014) }\end{array}$ & 65 & 64,2 (mean) & $35,70^{\circ}$ & FH \\
\hline
\end{tabular}

mean $=$ Mittelwert,$\quad \mathrm{FH}=$ Frankfurter Horizontale,$\quad \mathrm{CE}=$ Camper-Ebene,$\quad \mathrm{SKN}=$ mittlere $\quad$ sagittale Kondylenbahnneigungswinkel 


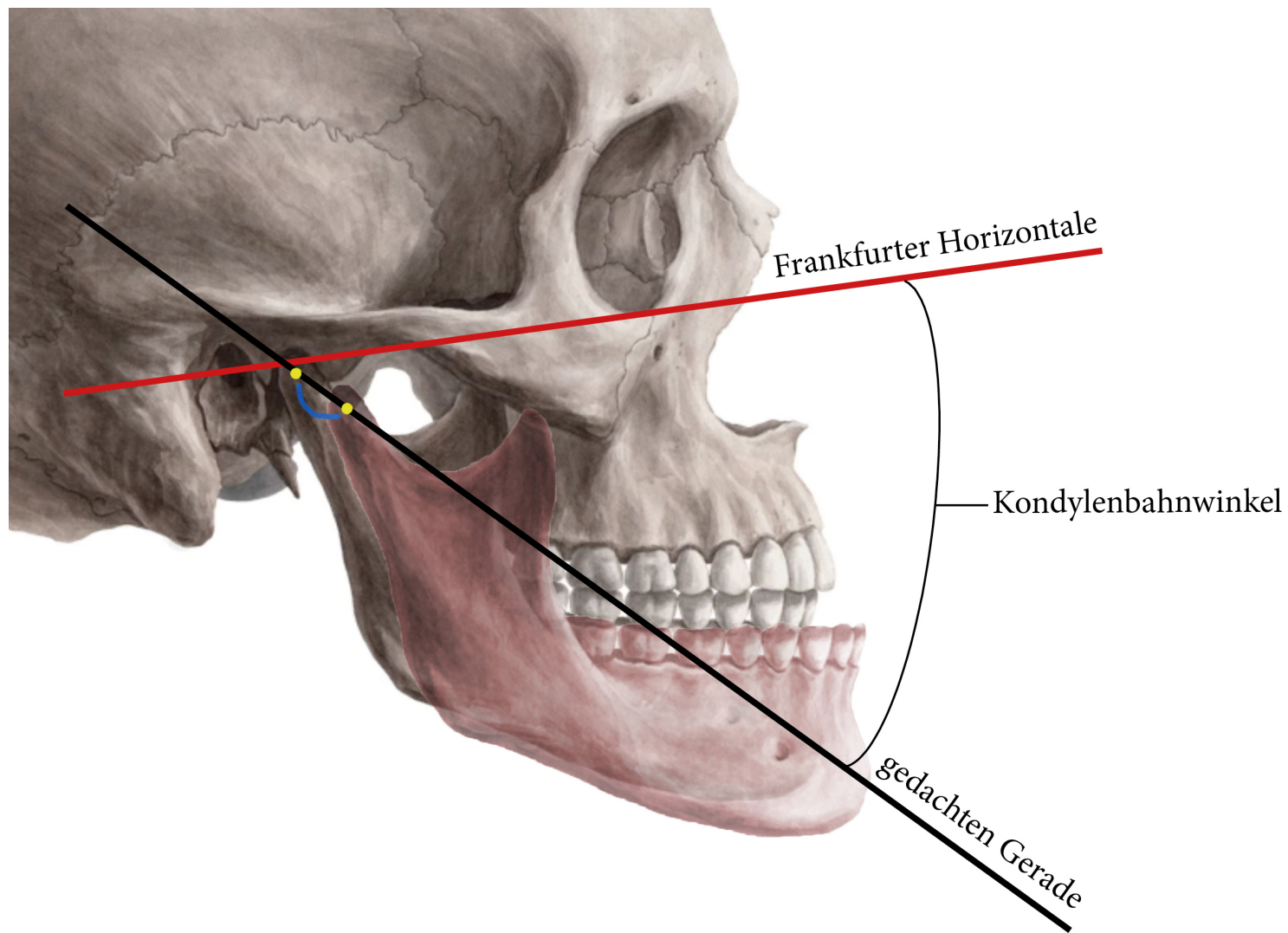

Abbildung 6: Kondylenbahnwinkel mit der Frankfurter Horizontalen als Referenzebene: protrudierter Unterkiefer in Rot, bogenförmige Kondylenbahn in Blau Modifiziert nach Yousun Koh (Koh 2020d) mit freundlicher Genehmigung von Kennhub

\subsubsection{Bennett-Winkel}

Transversale Kondylenbahnen lassen sich bei der Lateralbewegung des Unterkiefers erfassen. Die Mediotrusionsbahn, welche bei der Verlagerung des Mediotrusionskondylus entsteht, bildet mit der Sagittalebene einen Winkel, den man als Bennett-Winkel (BA) bezeichnet (Sander et al. 2011). Starten bei der Laterotrusion diese unterschiedlichen Bewegungen der Kondylen nicht synchron, dann kann man den „,immediate side shift" (ISS) registrieren (siehe Abbildung 7). Dabei führt der Mediotrusionskondylus zu Beginn der Bewegung einen kleinen Seitenversatz nach medial durch, bevor dieser nach vorne wandert (Bumann und Lotzmann 2000). Der BA und der ISS sind - genauso wie die sagittale Kondylenbahnneigung - wichtige Größen für die Artikulatorprogrammierung und beeinflussen mit ihrer Größe die anatomische Gestaltung der Höcker im Seitenzahnbereich sowie die Konkavität der Palatinalflächen der Oberkieferfrontzähne bei der Herstellung von festem und partiellem Zahnersatz (Wenz und Hellwig 2018). Eine Übersicht der durchschnittlichen Bennett-Winkel aus der Literatur mit den jeweiligen Bezugsebenen ist in Tabelle 2 aufgebführt. 
Tabelle 2: Literaturübersicht über Untersuchungsergebnisse zur Messung des Bennett-Winkels

\begin{tabular}{|c|c|c|c|}
\hline Autor und Jahr & $\begin{array}{c}\text { Anzahl der } \\
\text { ProbandInnen }\end{array}$ & Methode & Ergebnisse/Mittelwert \\
\hline $\begin{array}{l}\text { Gysi } \\
(1929)\end{array}$ & 18 & Gysi-Bogen & $5-25^{\circ} / 15^{\circ}$ \\
\hline $\begin{array}{l}\text { Fischer } \\
(1959)\end{array}$ & 116 & Gysi-Bogen & $0-30^{\circ}$ \\
\hline $\begin{array}{l}\text { Lundeen et al. } \\
\text { (1973) }\end{array}$ & 50 & Lee-Recorder & $8^{\circ}$ \\
\hline $\begin{array}{l}\text { Lauritzen et al. } \\
\text { (1974) }\end{array}$ & 50 & $\begin{array}{c}\text { Laterale } \\
\text { Wachsregistrate }\end{array}$ & $0-45^{\circ} / 23^{\circ}$ \\
\hline $\begin{array}{l}\text { Koeck et al. } \\
\text { (1976) }\end{array}$ & 56 & $\begin{array}{c}\text { Denar- } \\
\text { Pantograph }\end{array}$ & $6-27^{\circ} / 23^{\circ}$ \\
\hline $\begin{array}{l}\text { Utz et al. } \\
\text { (1987) }\end{array}$ & 123 & $\begin{array}{l}\text { Paraokklusale } \\
\text { Achsiographie }\end{array}$ & $\begin{array}{c}0-61^{\circ} / 8^{\circ} \text { bei Zahnführung } \\
15^{\circ} \text { bei seitlichem Druck }\end{array}$ \\
\hline $\begin{array}{l}\text { Lückerath } \\
\text { (1991) }\end{array}$ & 60 & Cardix-System & $\begin{array}{l}16,2^{\circ} \text { Rechts } \\
13,5^{\circ} \text { Links }\end{array}$ \\
\hline
\end{tabular}

Diese Darstellungen werden jedoch der komplexen dreidimensionalen Gelenkstruktur nicht gerecht, denn sie vernachlässigen die Formvariabilität der Kondylen, die asymmetrische Konkavität der Fossa articularis sowie die unterschiedliche Winkelbildung zwischen den kondylären Hauptachsen (Sander et al. 2011). Dadurch entsteht der Eindruck, dass das Kiefergelenk ein gleitendes Scharniergelenk mit einer Rotationsachse ist, welches durch seine beiden Kondylen geführt wird (Bowley und Pierce 1990; Hayashi et al. 1994). Neue Analyse- und Registriertechniken haben gezeigt, dass bei den Öffnungs- und Schließbewegungen des Unterkiefers die Rotationsachse nicht räumlich fixiert ist (Ferrario et al. 1996). Sie verläuft nicht durch die Kondylen, sondern viel eher im Bereich des Kieferwinkels und variiert mit der Translationsrichtung des Unterkiefers (Gallo et al. 2006). 


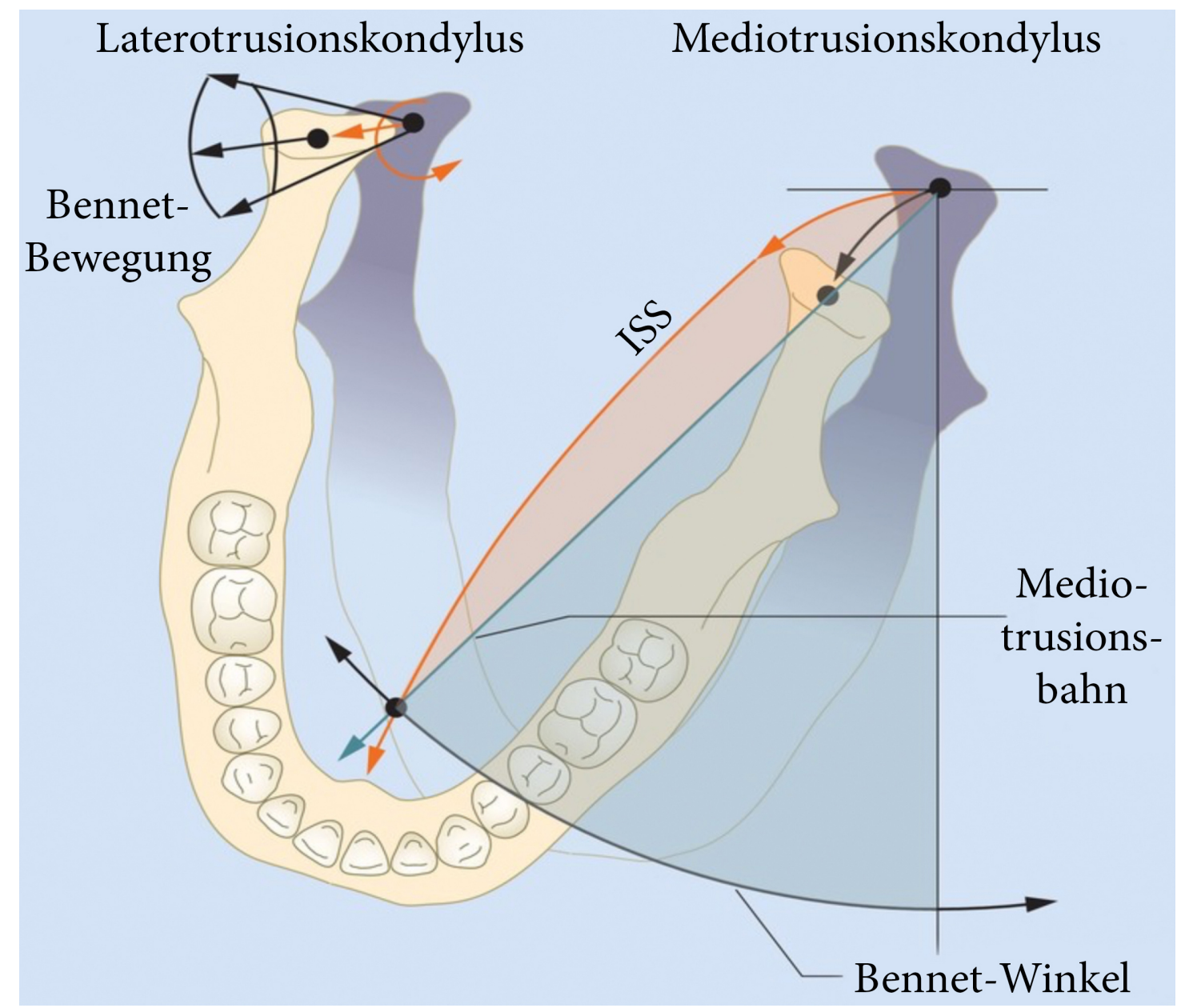

Abbildung 7: Versatz der Kondylen bei Laterosionsbewegung Bennett-Winkel zwischen der Mediotrusionsbahn und der Sagittalebene:

ISS = immediate side shift (rot)

Modifiziert nach Sander et al. (Sander et al. 2011) mit freundlicher Genehmigung des Georg Thieme Verlag

\subsubsection{Instrumentelle Bewegungsanalyse}

\subsubsection{Definition}

Unter der instrumentellen Funktionsanalyse ordnen sich verschiedene Messverfahren und Untersuchungstechniken zur patientenindividuellen Ermittlung der Unterkieferbewegungen und Kondylenposition ein, welche eine bessere Beurteilung der Funktion des craniomandibulären Systems ermöglichen (Kordaß und Mundt 2003). Diese setzt sich hauptsächlich aus der instrumentellen Bewegungsanalyse des Unterkiefers, der Kondylenpositionsanalyse, der horizontalen Kieferrelationsbestimmung sowie der Untersuchung der Kaumuskulaturaktivität zusammen (Utz et al. 2016). Der Einsatz von speziellen Registriersystemen bei den instrumentellen Bewegungsanalysen ermöglicht die Erfassung der Unterkiefer-Bewegungsfunktion eines/einer PatientIn. Die Bewegungsaufzeichnungen können anschließend analysiert und ausgewertet werden. 
Daraus werden Schlussfolgerungen gezogen, die sowohl die funktions- und strukturbezogene Diagnostik des Kauapparats betreffen als auch die Therapieplanung und Therapiegestaltung in der zahnärztlich-restaurativen Rehabilitation (Yatabe et al. 1995). Die Registriersysteme erfassen Unterkieferbewegungen mit zahngeführten und nichtzahngeführten Bewegungsabschnitten auf kinematischer Grundlage (Ettlin und Gallo 2018). Mit Hilfe eines Artikulators, der die okklusalen Verhältnisse anhand montierter Kiefermodelle darstellt, können statische und dynamische Okklusionsanalysen gemessen und registriert werden. Viele moderne Computerprogramme erlauben auch die virtuelle Simulation der dynamischen und statischen Okklusion und ermöglichen so zunehmend digitale Diagnoseverfahren und erleichtern die computergestützten Konstruktions- und Fertigungsverfahren (CAD/CAMVerfahren) (Gernet et al. 2007; Wieckiewicz et al. 2014).

\subsubsection{Nutzen}

Schon zu Beginn des 20. Jahrhunderts haben sich viele namhafte Wissenschaftler (z. B. Gysi, Stuart, McCollum, Schröder oder Gerber) bemüht, mechanische Registriersysteme zu entwickeln, um die Bewegungen des Unterkiefers aufzuzeichnen (Meyer 1993; 1996; Hugger 2000). Diese Aufzeichnungen dienten dazu, die Passung und Qualität zahnärztlich-restaurativer Arbeiten zu verbessern, indem die okklusale Gestaltung des Zahnersatzes individuell auf die/den PatientIn (und seine/ihre Unterkieferbewegungen) adaptiert wurde (Bumann und Lotzmann 2000).

Mitte der 1970er Jahre wurden die ersten Studien durchgeführt, die sich mit der Beweglichkeit und Koordination des Unterkiefers beschäftigten (Lewin und Ramadori 1985; Jankelson 1990). In der Zeit wurden viele Kenntnisse über die Funktionsstörungen des Kiefergelenkes gewonnen, die dann auch zur Behandlung der craniomandibulären Dysfunktionen okklusale Interventionen vorsahen (Rammelsberg 1998). Bis heute wurden viele Messsysteme kontinuierlich weiterentwickelt, um die in den 1970er und 1980er Jahren mechanisch aufzeichnenden Systeme durch elektrische Registriersysteme zu ersetzen (Schierz und Reissmann 2008a). Diese sollten praxistauglicher sein und den damals hohen apparativen, personellen und zeitlichen Aufwand der mechanischen Messsysteme reduzieren und gleichzeitig die Reliabilität und Genauigkeit der Messungen erhöhen (Utz et al. 2016).

Aktuell verfügbare elektronische Registriersysteme ermöglichen unabhängig von ihren Messtechnologien (elektrische, akustische, optische oder taktile Signale) die individuelle 
und digitale Erfassung bzw. Auswertung der Unterkieferbewegungen (Engelhardt 1993; Kordaß 2002; Stelzenmüller und Wiesner 2010).

\subsubsection{Messprinzipien}

Das Anbringen von Messsensoren direkt am Kiefergelenk ist nicht möglich, was wiederum die Analyse und Beurteilung der Bewegungsbahnen der Kondylen erheblich erschwert (Schierz und Reissmann 2008b). Deshalb werden sämtliche praxistauglichen Messsysteme, die für die Ermittlung relevanter diagnostischer Informationen über den Funktionszustand des Kiefergelenks verwendet werden, abhängig von ihren Messprinzipien kategorisiert (Schmitter et al. 2011). Systeme, die während der Messung mit den entsprechenden Sensoren in enger Lagebeziehung zum Kiefergelenk arbeiten, werden in die Gruppe der gelenknahen Messsysteme zusammengefasst. Andere, die in der Regel ventral des Patientengesichtes messen, werden in die Gruppe der gelenkfernen Messsysteme eingeteilt (Hugger 2000). Zudem lassen sich die Systeme bezüglich ihres Kontaktes von Signalsender und -empfänger in berührungshaft oder berührungslos einordnen (Schmitter et al. 2011).

\subsubsection{Artikulatorprogrammierung}

\subsubsection{Die Achsiographie}

Die Registrierung von Unterkieferbewegungen zur Gewinnung patientenindividueller Daten der Kondylenbewegungen und der Frontzahnführung wird unter Anwendung von kinematischen Messverfahren als Achsiographie bezeichnet (Wilm-Gert 2006). In den letzten Jahren haben die modernen elektronischen Messsysteme die in der Vergangenheit rein mechanischen Apparaturen abgelöst. Die bei der Achsiographie gewonnenen Bewegungsdaten des Unterkiefers sind die Grundlage für eine patientenindividuelle Einstellung („Programmierung“) von Artikulatoren, um eine nahezu exakte Simulation der Unterkieferbewegungen des PatientIn im zahntechnischen Labor zu ermöglichen (Utz et al. 2016). Ohne die individuelle Artikulator-Programmierung werden zahntechnische Restaurationen in sogenannten Mittelwertartikulatoren hergestellt, bei denen keine patientenindividuelle Programmierung möglich ist (Schmitter et al. 2011). Dies kann entweder zu erhöhten Einschleifmaßnahmen beim Eingliedern des Zahnersatzes aufgrund starker Interferenzen führen oder zur Entstehung von überdimensionierten interokklusalen Freiräumen, die für eine ineffiziente Verzahnung im biomechanischen Sinne und damit für eine reduzierte Kaueffektivität verantwortlich sind (Pröschel et al. 
2000; Schindler und Hugger 2008). Um eine möglichst störungsfreie strukturelle und funktionelle Integration von Zahnersatz in das craniomandibuläre System zu ermöglichen, ist eine individuelle Programmierung von Artikulatoren empfehlenswert (DGPro 2010).

\subsubsection{Gesichtsbogen}

Die dreidimensionale Lagebeziehung der Okklusionsebene (des Oberkiefermodells) zu den entsprechenden Bezugsebenen am Schädel kann durch die Anwendung eines Gesichtsbogens in den Artikulator übertragen werden (Craddock und Symmons 1952). Dabei ist es wichtig, den zu dem verwendeten Gesichtsbogen passenden Artikulator zu benutzen, da die Bezugsebenen auf das jeweilige Artikulator-System abgestimmt sind und die Verwendung fremder Komponenten zu Fehlern in der Modellmontierung im Artikulator führt (Schunke 2012).

Der Nutzen des Gesichtsbogens ist in der internationalen Literatur umstritten. Im skandinavischen Raum kommen Gesichtsbögen kaum zum Einsatz, weil der Nutzen der vertikalen Positionierung der Modelle im Artikulator mit Hilfe eines Gesichtsbogens für funktionsanalytische oder prothetisch-restaurative Maßnahmen angezweifelt wird (Hugger et al. 2001). Im Vergleich zu dieser eher ablehnenden Haltung wird vor allem in vielen englisch- und deutschsprachigen Lehrbüchern auf die Notwendigkeit zur Verwendung eines Gesichtsbogens - vor allem bei komplexen prothetischen Rehabilitationen - hingewiesen. Dies wird mit der Individualisierung von Unterkieferbewegungen und der daraus resultierenden Verbesserung der Qualität von Restaurationen durch die präzisere Modelmontage in den Artikulator begründet. Während beispielsweise an zehn von zwölf Zahnkliniken in Irland und Großbritannien (wie an den meisten deutschen Universitätskliniken) die Verwendung des Gesichtsbogens im Rahmen des Zahnmedizinstudiums gelehrt wird (Lynch et al. 2010), wird in Schweden davon ausgegangen, dass die mittelwertige Modellmontage in den Artikulator ohne Gesichtsbogen ausreichend ist (Carlsson und Magnusson 2000).

Für die regelrechte Montage des Oberkiefermodells im Artikulator müssen mindestens zwei räumliche Referenzebenen definiert werden. Die Okklusionsebene des Oberkiefers und der Abstand der Oberkieferzähne zur Scharnierachse werden als zwei - in posterioranteriorer Richtung - voneinander unabhängige Lagebeziehungen ermittelt und durch den Gesichtsbogen in den Artikulator übertragen (Ellis et al. 1992). Laut der Deutschen Gesellschaft für Prothetische Zahnmedizin und Biomaterialien (DGPro) und der 
Deutschen Gesellschaft für Funktionsdiagnostik und -therapie (DGFDT) wird die Okklusionsebene als eine Ebene bezeichnet, die durch den Kontaktpunkt der mittleren Inzisivi des Unterkiefers und durch die disto-bukkalen Höcker der zweiten Unterkiefer Molaren verläuft (Ahlers et al. 2006). Die Scharnierachse ist eine gedachte Linie zwischen den rechten und den linken Kondylus und verläuft imaginär am Schädel weiter durch die Weichteile (Sörgel 1978). Diese sogenannte Interkondylarlinie bildet eine funktionelle Drehachse bei der Rotation beider Kondylen beim Öffnen und Schließen des Unterkiefers (siehe Abbildung 8) (Ahlers et al. 2006).

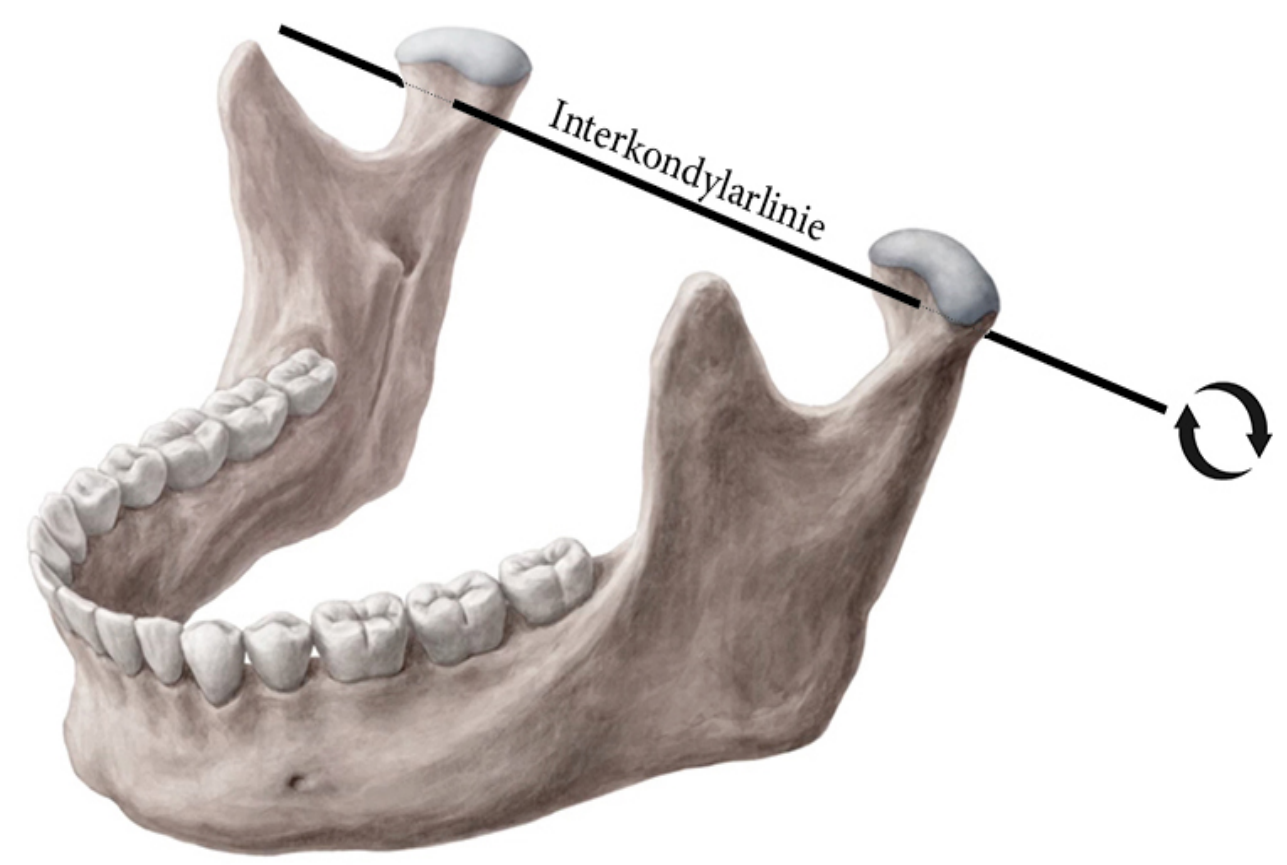

Abbildung 8: Mandibula-Drehachse

Modifiziert nach Yousun Koh (Koh 2020c) mit freundlicher Genehmigung von Kennhub

\subsection{Kinematische Scharnierachse}

Die individuelle Lokalisation der kinematischen Scharnierachse kann mechanisch oder elektronisch für jede(n) PatientIn erfolgen (Utz et al. 2016). Beide Verfahren stellen den Versuch dar, durch die Rotationsbewegung beider Gelenkköpfe bei geringer Mundöffnungs- und Mundschließungsbewegung, einen kondylären Punkt zu bestimmen, der die Eigenschaft einer translatorisch gleitenden Rotationsachse aufweist, um dadurch die Kinematik des gesamten Kondylus zu simulieren (Ahlers et al. 2006). Mechanisch wird dafür in der Regel ein Registrierbogen im Unterkiefer befestigt, der mit Hilfe seiner Seitenarme im Bereich beider Kondylen die Rotationsachse beim initialen Öffnen und 
dem terminalen Schließen lokalisiert (Posselt und Odont. 1958; Lotzmann 1990). Gegenüber dieser kinematischen Scharnierachsenbestimmung, die auch als „wahre“ Scharnierachse bezeichnet wird, steht die arbiträre Scharnierachse (Ellis et al. 1992).

\subsection{Arbiträre Scharnierachse}

Weil die patientenindividuelle Ermittlung der kinematischen Scharnierachse sehr zeitaufwendig ist, wird bei praxisalltäglichen Behandlungsabläufen häufig die wesentlich schneller und einfacher zu bestimmende arbiträre Scharnierachse verwendet (Simpson et al. 1984). Hier wird ein mittelwertiger Punkt bestimmt, in dessen Umkreis sich die kinematische Scharnierachse mit hoher Wahrscheinlichkeit nachweisen lässt. Eine Fehllokalisation von bis zu $5 \mathrm{~mm}$ zur kinematischen Scharnierachse ist wahrscheinlich klinisch vernachlässigbar und erlaubt eine ausreichend genaue Positionierung von Oberkiefermodellen im Artikulator ohne wesentliche Abweichungen bei der okklusalen Gestaltung von Zahnersatz (Årstad 1954; Weinberg 1959; Singh et al. 2017). Eine größere Diskrepanz zwischen der arbiträren und kinematischen Scharnierachse kann jedoch zu deutlichen okklusalen Fehlern bei der Anfertigung von Zahnersatz führen (Piehslinger et al. 1995; Celar und Tamaki 2002).

Die Bestimmung der arbiträren Scharnierachse kann entweder durch die Markierung eines Referenzpunktes auf einer gedachten Verbindungslinie vom Porus acusticus externus zum äußeren Augenwinkel oder durch die Verwendung eines Gesichtsbogens mit sogenannter Ohr-Olive (Ohr-Gesichtsbogen) erfolgen (Walker 1980; O’Malley und Milosevic 2000; DGPro 2010). In der Literatur variieren die Angaben über den Abstand dieses Scharnierachsenpunktes vom posterioren Referenzpunkt (Porus acusticus externus) zwischen 10 bis 13 mm (Abdal-Hadi 1989). In den zahnärztlichen Praxen werden eher die Ohr-Gesichtsbögen verwendet (Ahlers 1996). Diese können mit Hilfe von zwei Ohr-Oliven sowie ihrer Bezugsebene die räumliche Lage der Oberkieferdentition zum Porus acusticus externus realisieren. (Goska und Christensen 1988; Leukhardt 2010).

Obwohl die Genauigkeit der mittelwertigen arbiträren Scharnierachse und die Auswirkungen auf die Herstellung von Zahnersatz in vielen Studien kontrovers diskutiert wird, wird diese in der praktischen Anwendung am Patienten jedoch in der Regel als ausreichend angesehen (Ahlers 1996). 


\subsubsection{Bezugsebenen}

Für die Montage eines Oberkiefermodells in den Artikulator wird zu den beiden posterioren Referenzpunkten (kinematische oder arbiträre Scharnierachse) ein weiterer anteriorer Referenzwert benötigt, um gemeinsam eine horizontale Referenzebene zu bilden (Weinberg 1961). Die Übertragung der dreidimensionalen Lage des Oberkiefers in den Artikulator wird durch diese Referenzebene ermöglicht, wobei die Neigung dieser Ebene somit von der Wahl des anterioren Referenzpunktes abhängig ist (O'Malley und Milosevic 2000).

\subsection{Frankfurter Horizontale}

Die Frankfurter Horizontale wurde erstmals 1882 auf einen Anthropologen-Kongress in Frankfurt vorgestellt und ist bis heute die Referenzebene, die am häufigsten bei der Gesichtsbogenübertragung verwendet wird (Krueger und Schneider 1986; Ellis et al. 1992). Durch die Verbindung der äußeren Traguspunkte (posteriorer Referenzpunkt) mit dem Infraorbitalrand (anteriorer Referenzpunkt) entsteht eine gedachte Ebene (Bumann und Lotzmann 2000). Diese dient der Übertragung des Oberkiefermodells in der gleichen Relation zum oberen Anteil des Artikulators und entspricht der Oberkieferlage des jeweiligen Patienten zu seiner Frankfurter Horizontalen (siehe Abbildung 9) (Seifert et al. 2000; The Glossary of Prosthodontic Terms: Ninth Edition 2017).

\subsection{Camper-Ebene}

Die im 18. Jahrhundert von dem niederländischen Anatom Pieter Camper eingeführte Ebene findet ebenfalls in der Anthropologie ihren Ursprung (Bojanov et al. 1972). Die Camper-Ebene verläuft durch die Spina nasalis anterior und die äußeren Traguspunkte und wird in der Literatur häufig als zur Okklusionsebene parallel verlaufend beschrieben (Bumann und Lotzmann 2000). In der zahnärztlichen Prothetik gilt deshalb die CamperEbene als Schädelbezugsebene zur Okklusionsebene und wurde daher historisch gesehen beim Gysi- oder Hanau-Artikulator favorisiert (siehe Abbildung 9) (Gärtner 2003). 


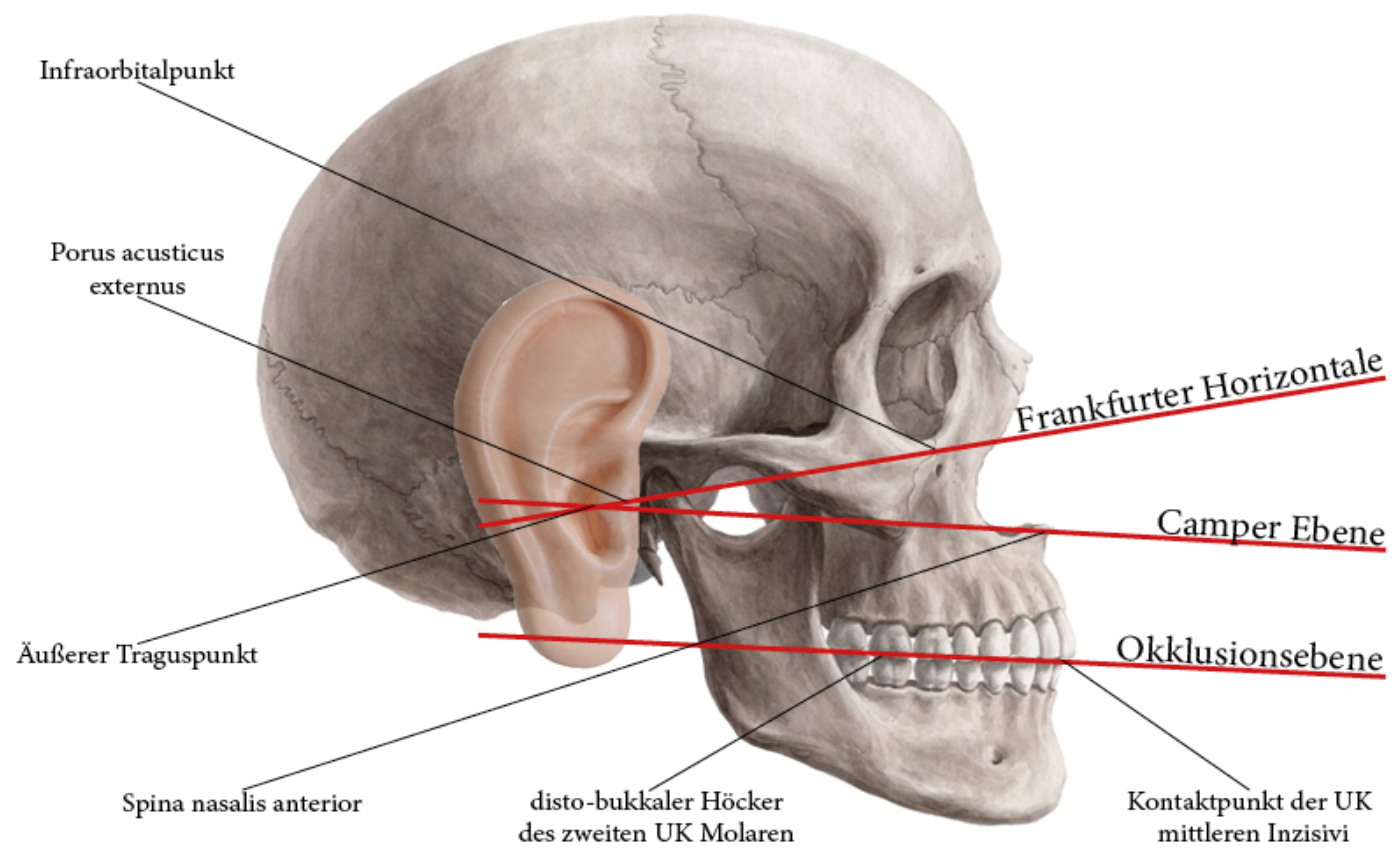

Abbildung 9: Referenzebenen

Modifiziert nach Yousun Koh (Koh 2020d) mit freundlicher Genehmigung von Kennhub

\subsubsection{Elektronische Systeme zur Unterkieferaufzeichnung}

\subsubsection{Entwicklung}

Die ersten elektronischen Messsysteme wurden in den 50er Jahren des letzten Jahrhunderts vorgestellt und dienten damals der grafischen Darstellung von Zahnkontakten durch einen Stromkreis, der sich bei Kontakt der Zähne schloss und entsprechende Signale wiedergab.

Zur Registrierung der Okklusion wurden zwei Spulen verwendet, die am Oberkiefer und am Unterkiefer fixiert wurden. Diese Methode wurde von Yurkstas und Emerson (1954), Brewer und Hudson (1961), Graf und Zander (1963) sowie Neill (1976) weiterentwickelt, sodass es 1969 Glickman et al. gelang ein Registriersystem vorzustellen, welches fünf verschiedene okklusale Positionen differenzieren konnte (Glickman et al. 1969).

Die ersten elektronischen Systeme, die das Registrieren von Unterkieferbewegungen erlaubten, wurden in den 1960er Jahren entwickelt. Bewersdorff führte 1967 mit seiner Apparatur die Elektrognathographie ein. Diese wurde über ein Seilsystem aufgehängt und registrierte die dreidimensionalen Unterkieferbewegungen berührungslos über drei Messaufnehmer (Bewersdorff 1967). 
Im Jahr 1970 entwickelte die Forschungsgruppe um Knap ein berührungshaftes Registriersystem, welches mit Hilfe von sechs Potentiometern, die paraokklusal befestigt wurden, eine gelenkferne dreidimensionale Messung in allen Freiheitsgraden ermöglichte (siehe Abbildung 10) (Knap et al. 1970).

Nur ein Jahr später präsentierte Körber ein auf Widerstandsmessung beruhendes System, mit dem zeitliche Längenänderungen sowohl gelenknah als auch gelenkfern erfasst werden konnten. Die Daten wurden von insgesamt neun Messwertaufnehmern, die Körber im Kiefergelenk- und Frontzahnbereich montierte, erfasst (Körber 1971).

Der im Jahr 1975 von Goodson und Johansen vorgestellte „Mandibulograph“ war das erste System, welches einen Computer zur mathematischen Registrierung der räumlichen Unterkieferbewegungsbahn verwendete. Für dieses Verfahren war es nötig, den Kopf des Probanden mit einer Gipsschale am Behandlungsstuhl zu fixieren, um die Messungen ohne äußere Bewegungseinflüsse durchzuführen (Goodson und Johansen 1975).

Luckenbach stellte im Jahr 1981 das „Elektronische Computergesteuerte Registriersystem“ (ECRS) vor. Dabei platzierte er gelenknah sieben Drehwinkelgeber, die jede beliebige Position eines Punktes im Ober- und Unterkiefer relativ zur Messapparatur berührungshaft bestimmen konnten (Luckenbach 1983).

Das „Pantoskop“ führte Ohlrogge 1982 ein. Dieses optoelektronische System registriert durch die im Behandlungsstuhl integrierten Sensoren gelenknah und berührungslos. Über einen Computerbildschirm wurden die Bewegungsspuren graphisch dargestellt (Ohlrogge 1982).

Der „String-LR-Recorder“ wurde bereits 1981 von Klett vorgestellt. Dieses optoelektronische und somit berührungslose Gerät beruhte auf einem Reflektorsystem, welches am Oberkiefer der PatientInnen starr befestigt wurde. Die drei Messköpfe mussten nach der individuellen Scharnierachsenbestimmung gelenknah über extraorale mit dem Unterkiefer verbundene Apparaturen montiert werden. Die entfernungsabhängige Lichtintensität wurde vom System empfangen und in Spannungssignale umgewandelt. Der „String-Condylocomp LR2“ als Nachfolger verfügte über paraokklusale Löffel für die Fixierung des Systems und besaß eine Messfehlerkorrektur. Klett präsentierte 1983 den „String-Condylocomp LR3“ mit einigen Neuerungen (siehe Abbildung 10). So hatte dieser nur noch zwei Messköpfe, die am Oberkieferbogen befestigt wurden und der paraokklusale Löffel des Unterkiefers trug die Reflektoren (Klett 1982a; 1983). Dazu brachte Edinger zur Darstellung und 
Untersuchung der Messdaten eine graphische Bedienoberfläche heraus, welche auf der inzwischen weit verbreiteten Windows Benutzeroberfläche basierte (Edinger 1990; Hugger et al. 1996).

Mochizuki und Hobo veröffentlichten 1983 ein Registriersystem, mit dem die Unterkieferbewegungen in sechs Freiheitsgraden gemessen werden konnten. Die dafür im Oberkiefer befestigten Leiterplatten standen mit den drei Griffeln der paraokklusalen Bissgabel in Verbindung und lieferten eine Messgenauigkeit von $\pm 0,06 \mathrm{~mm}$ (Hobo und Mochizuki 1983).

Meyer und dal Ri erweiterten 1985 einen mechanischen Axiographen, indem sie spezielle Widerstandsfolien anstelle der Schreibplatten verbauten und die mechanische Messuhr gegen induktive Taster tauschten. Die Reproduzierbarkeit der Messungen sowie die Messgenauigkeit erhöhten sich hierdurch (Meyer und dal Ri 1986).

Die Untersuchungen von Pröschel zur Einteilung der Kaubewegungen in verschiedene Kaubewegungsmuster führte er 1987 mit den von ihm entwickelten Elektrognathographen durch (Pröschel 1987; Pröschel und Hofmann 1987).

A

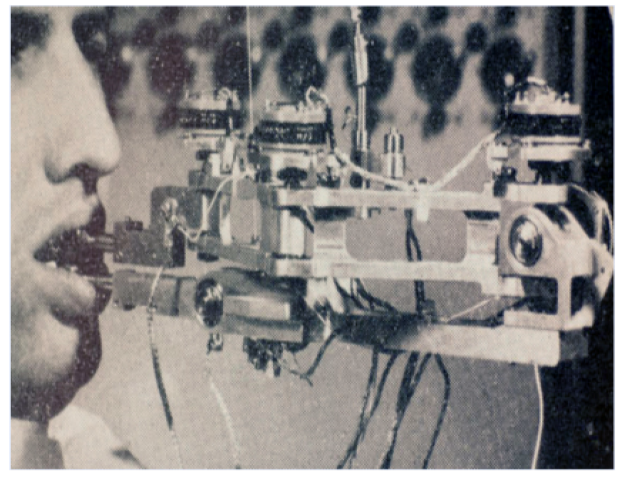

Registriersystem nach KNAP (Quelle: opus.bibliothek.uni-wuerzburg.de).

C

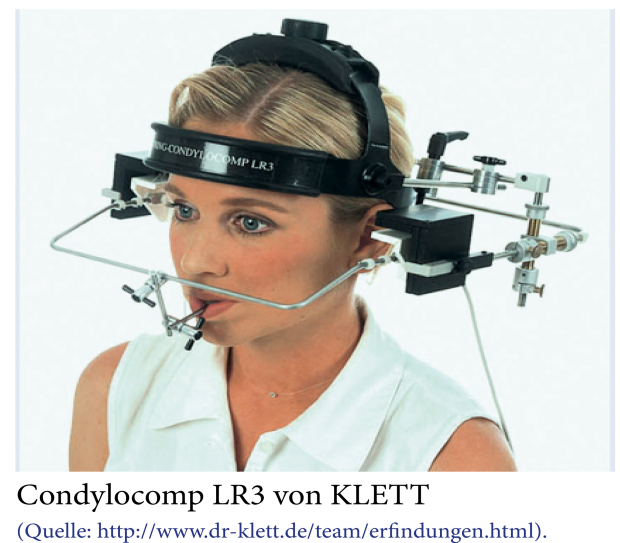

$\mathrm{B}$

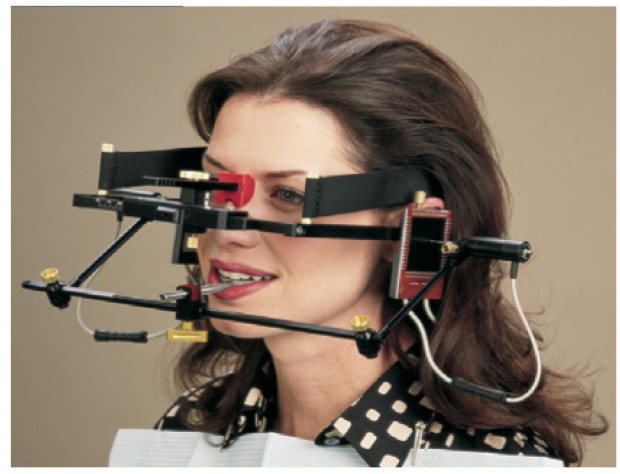

Cadiax $^{\oplus}$ Compact 2 von SLAVICEK (Quelle: http://shop.whipmix.com).

$\mathrm{D}$

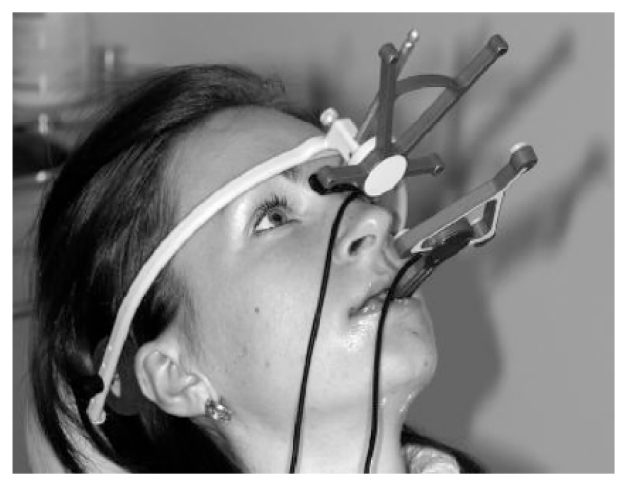

ARCUSdigma von der Firma KaVo (Quelle: Kobs et al. 2007).

Abbildung 10: Historische elektronische Registriersysteme 
Parallel zu Kletts „String-Condylocomp“ wurde der „Stereognatograph“ von Burckhardt vorgestellt. Dieses optoelektronische System arbeitete mit Hilfe von speziellen am Unterkiefer angebrachten Filterscheiben und am Oberkiefer montierten Messsensoren auf Basis des Transmissionsprinzips von Infrarotstrahlung (Burckhardt 1982; 1991; Burckhardt und Polz 1986).

Auf Basis der Sonographie entwickelte das Unternehmen von Dr. Hansen \& Co. mit ihrem „MT1602“ ein berührungsloses Messsystem mit drei Ultraschallsendern und Empfängern. Diese konnten gelenkfern die Unterkieferposition durch die Auswertung der Laufzeitdifferenzen in Echtzeit bestimmen. Die am paraokklusalen Löffel gelenkfern befestigten Empfänger- und Sendereinheiten waren jedoch schwingungsanfällig und die Registrierung war zudem gegenüber anderen Frequenzen im Raum sehr störanfällig (Klamt et al. 1990; Pröbster und Benzing 1990; Schrader 1994; Kordaß 1996).

Im Jahr 1990 konnte Edinger ein „Ultraschallregistriersystem“(URS) vorstellen, welches im Vergleich mit dem „MT1602“ eine bessere Auflösung aufweisen konnte und durch die gelenknahe Registrierung sichere Messungen lieferte (Edinger 1990).

Das von Slavicek mit der Firma GAMMA entwickelte „CADIAX-System“ war ein elektromechanisch aufzeichnendes Registriersystem mit zwei Gesichtsbögen (siehe Abbildung 10). Die gelenknahe Aufzeichnung erfolgte über zwei lateral angebrachte Flaggen und zwei elektrische Schreibstifte, welche die Bewegungen des Unterkiefers aufzeichneten (Schierz und Reissmann 2008b).

Heutige ultraschallbasierte Systeme beruhen, wie das „URS“ von Edinger, auf Laufzeitmessungen der Ultraschallsignale zwischen Sender und Empfänger. Um die Messgenauigkeit zu erhöhen, werden in den heute erhältlichen Registriersystemen mehrere Sensoren verbaut, um so die begrenzte Wellenlänge des angewendeten Ultraschalls zu optimieren (Schierz und Reissmann 2008b). Die Sensoren können aus Platzgründen jedoch nicht alle gelenknah positioniert messen, sodass eine Interpolation der Messwerte erfolgen muss. Dazu gehören Beispielsweise die Geräte „Jaw-MotionAnalysis“ und „SAM-Axioquick-Recorder“ der Unternehmen Zebris sowie der „ARCUSdigma“" von KaVo (siehe Abbildung 10) (Schierz und Reissmann 2008b).

\subsubsection{ARCUSdigma II}

ARCUSdigma II (digital movement analyzer) ist ein elektronisches Messsystem, welches von der Firma KaVo Dental im Jahre 2007 als Nachfolger des ARCUSdigma 
(Markteinführung 2001) eingeführt wurde (Wegmann 2009). Das Gerät arbeitet berührungslos auf Ultraschallbasis und kann gelenkfern Bewegungen des Unterkiefers registrieren und darstellen (Schmitter et al. 2011). Es besteht aus einem mandibulären (Sender) und einem maxillären Bogen (Empfänger), welche am Kopf des PatientIn befestig werden. Die acht im maxillären Bogen intergierten Empfängersensoren registrieren die Ultraschallwellen von den vier Sendern des mandibulären Bogens (Schierz und Reissmann 2008b). Der maxilläre Bogen bildet nach Kalibrierung eine feste Bezugsebene für den mandibulären Bogen, sodass die Laufzeitdifferenz der Ultraschallwellen berechnet werden kann. Dadurch kann das Gerät die dreidimensionale Bewegung des Unterkiefers aufzeichnen und schafft es durch die Integration mehrerer Sender Messfehler zu reduzieren. Über einen Monitor können sowohl die errechneten Bewegungsbahnen der Kondylen als auch die des Unterkiefer-Inzisalpunktes grafisch in Echtzeit dargestellt werden (Schierz und Reissmann 2008b). Die Bewegungsaufzeichnungen können am Computer in einer 3D-Symulation veranschaulicht werden und die Graphen und eine tabellarische Auflistung der Messwerte sind ebenso abrufbar (siehe Abbildung 11 und Abbildung 12).

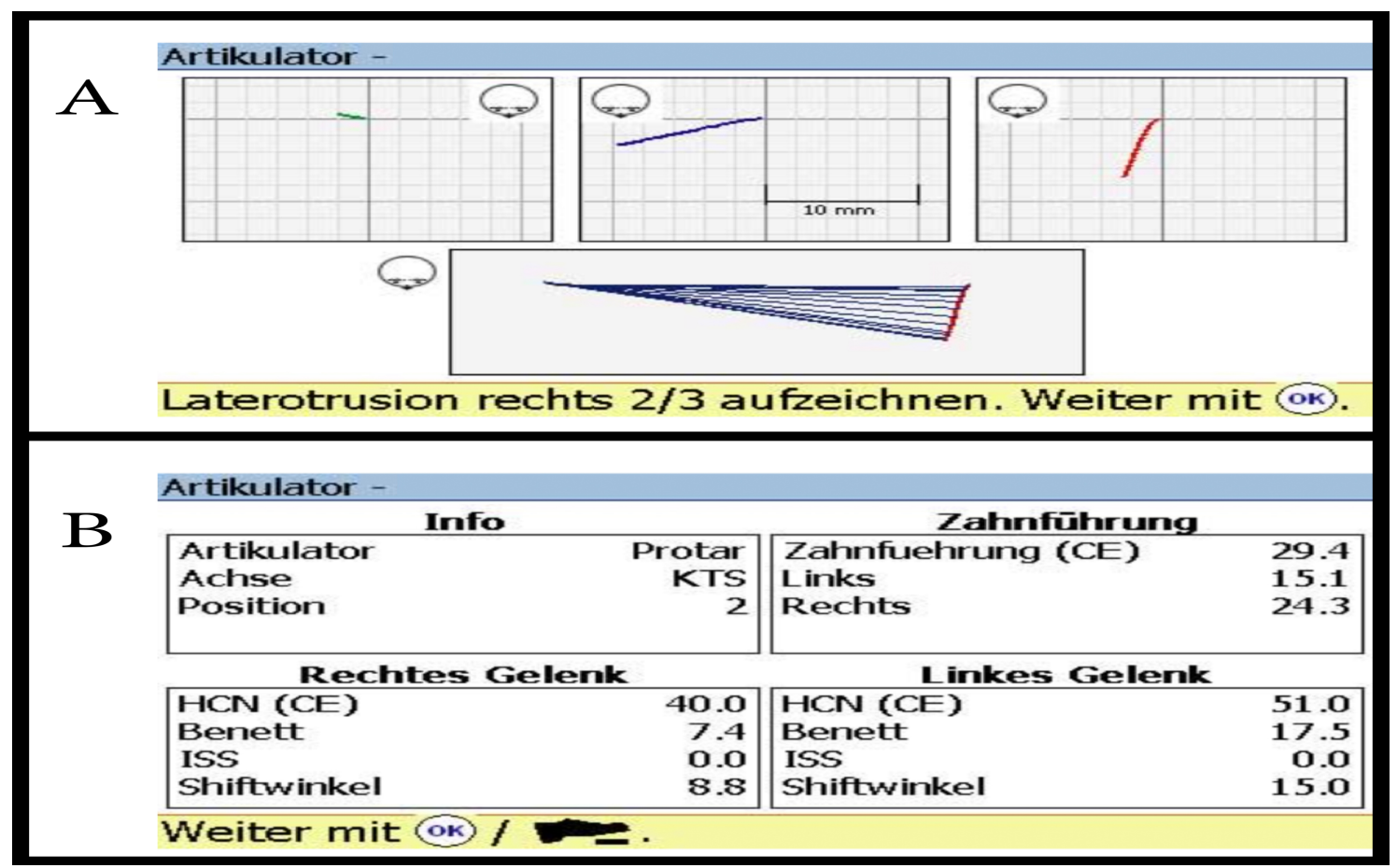

Abbildung 11:Datenerfassung und Darstellung auf dem ARCUSdigma-II-Monitor: $\mathrm{A}=$ Darstellung der Bewegungsbahnen der Kondylen, $\mathrm{B}=$ Automatisch ermittelte Werte zur Artikulatorprogrammierung 


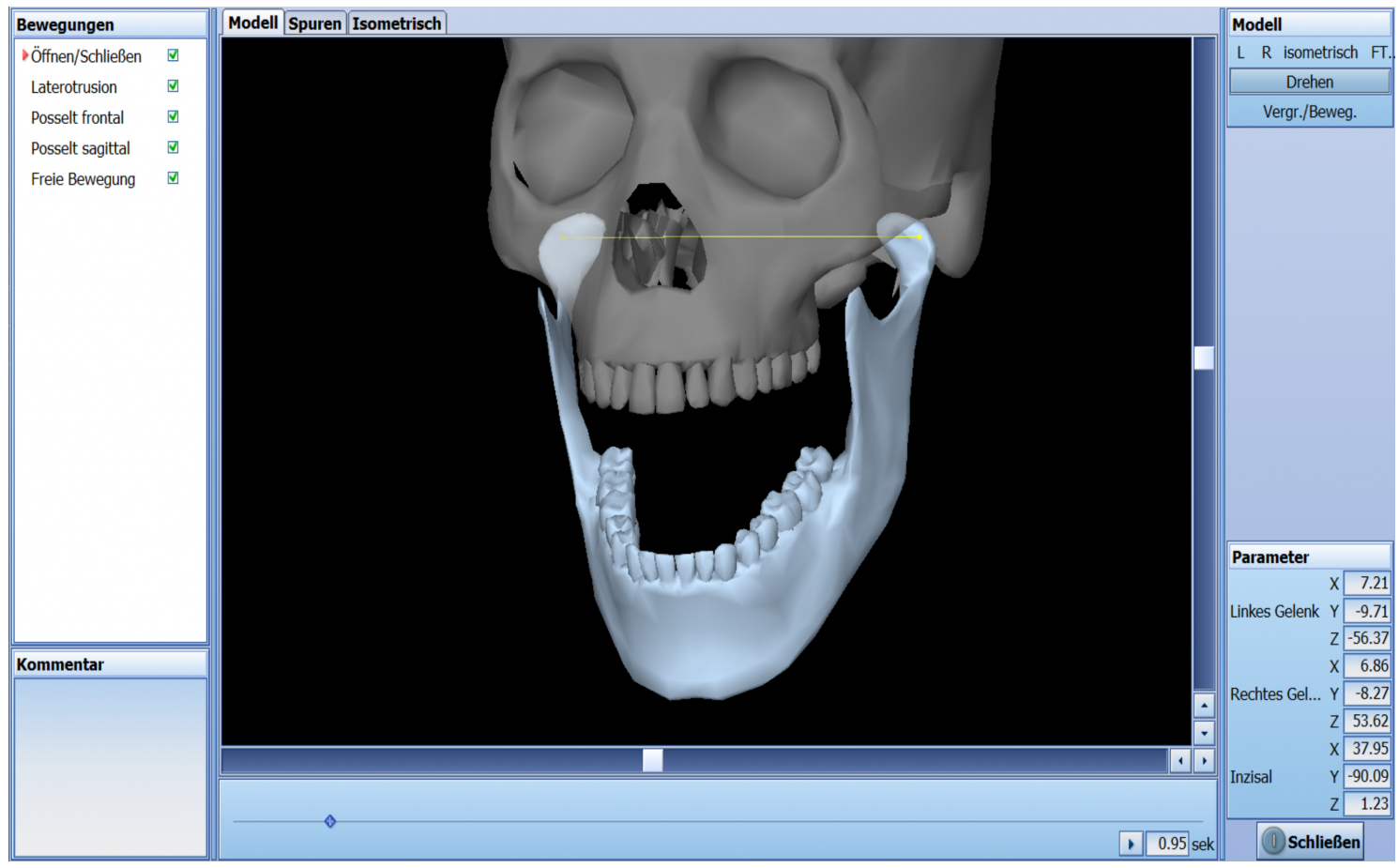

Abbildung 12: Dreidimensionale Darstellung der Unterkieferbewegungen als Video im ARCUSdigma II-Softwareprogramm

\subsection{Nutzen}

Das ARCUSdigma II liefert patientenindividuelle Daten für die Programmierung volljustierbarer Artikulatoren und bietet zusätzlich mit der KaVo-Transfer-System (KTS) Bissgabel eine schnelle und einfache Möglichkeit zur Übertragung dieser Parameter in den Artikulator (KaVo Dental GmbH 2012). Die Artikulatorgeometrie wird dafür virtuell in den Patientenschädel projiziert und die virtuellen Referenzpunkte werden passend zu den Artikulatorgelenken aufgezeichnet (Pröschel et al. 2002).

Neben der Artikulatorprogrammierung und der Bestimmung der zentrischen Kondylenposition erlaubt das System eine 3D-Bewegungsanalyse und bietet durch die Module EPA (Electronic Position Analysis) und EAEF (elektronische Analyse ätiologischer Faktoren) auch Diagnostik- und Therapieoptionen bei PatientInnen mit craniomandibulären Dysfunktionen an (Fasold et al. 2012). Potentialänderungen an Muskeloberflächen im Sinne der Elektromyographie abzuleiten, ist eine nicht invasive und schmerzfreie Möglichkeit zur Analyse der Kaumuskulaturaktivität, welche das EMG-Modul des ARCUSdigma II optional bietet (KaVo Dental GmbH 2012).

\subsection{Aufbau}

Außer den bereits erwähnten Bögen (Sender- und Empfängerbogen) verfügt das Gerät über einen Fußschalter, um die Aufzeichnung zu beginnen und $\mathrm{zu}$ beenden, ein 
Touchscreen-Basisgerät zur Ansicht der Bewegungsabläufe und zur Eingabe der Patientendaten, einen okklusalen Löffel zur Kalibrierung des Empfängerbogens mit der Oberkieferlage und einen paraokklusalen Löffel zur Befestigung des Senderbogens am Unterkiefer (siehe Abbildung 13) (KaVo Dental GmbH 2008).
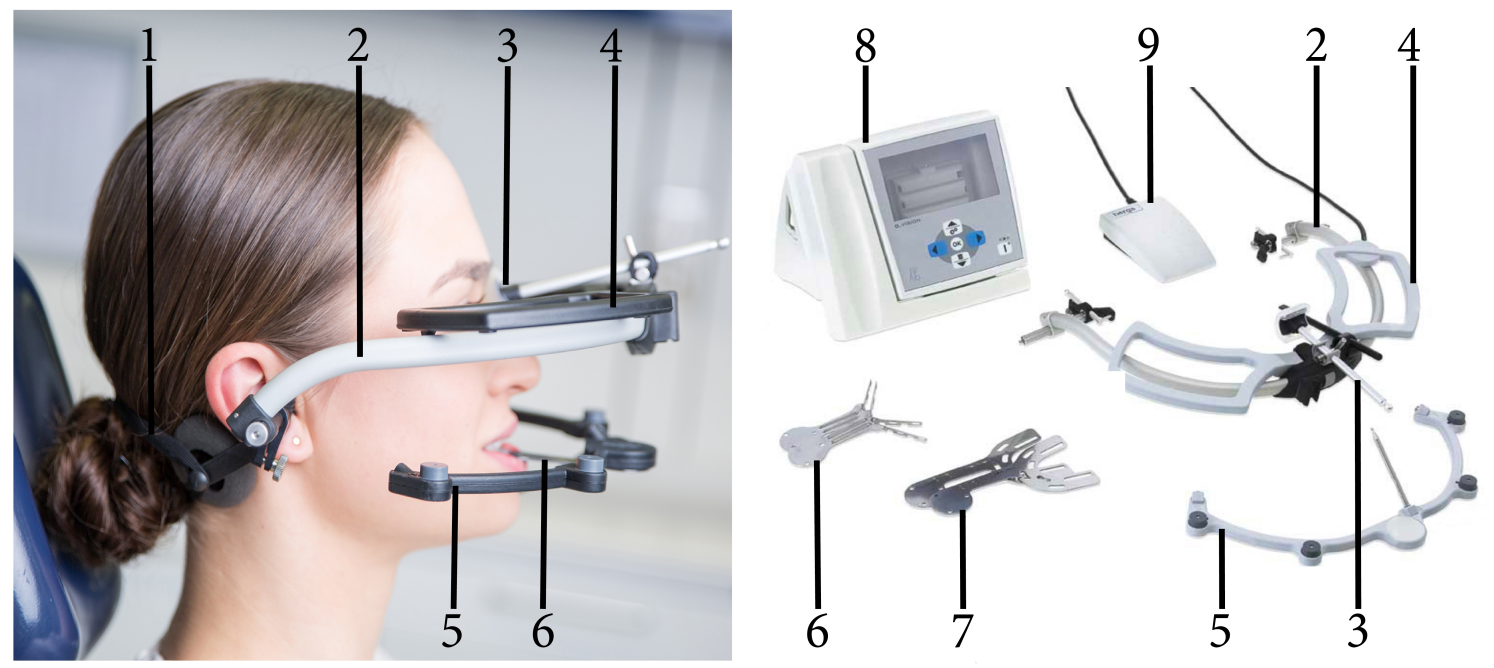

Abbildung 13:Komponenten des ARCUSdigma II:

$1=$ Hinteres Gummiband, $2=$ Maxillärer Bogen, $3=$ Nasenstütze,

4 = Empfänger, $5=$ Sender, 6 = Paraokklusale Löffel für den Unterkiefer, $7=$ KTS-Bissgabeln für den Oberkiefer, $8=$ Touchscreen Basisgerät, $9=$ Fußschalter

\subsection{Anlegen}

Die beiden Bögen des Gerätes werden am Kopf des aufrecht sitzenden Patienten fixiert. Der maxilläre Bogen (Empfänger-Bogen) wird an den äußeren Gehörgängen und der Nasenwurzel sowie mit Hilfe eines elastischen Gummibandes über eine Druckbefestigung am Hinterkopf angelegt (siehe Abbildung 13). Der Bogen verfügt über eine verstellbare Nasenstütze und ein Einstellrad, welche sich zur Orientierung an der Frankfurter Horizontale als Bezugsebene beim Anlegen verschieben lassen. Die rückwärtigen Gummibänder werden geschlossen, um den Sitz des maxillären Bogens über die ganze Messdauer zu sichern.

Der mandibuläre Bogen (Sender-Bogen) wird mit einem paraokklusalen Löffel an den Zähnen des Unterkiefers zur Bewegungsmessung fixiert. Die Befestigung des Empfänger-Bogens am Schädel sowie des Sender-Bogens paraokklusal an den Unterkieferzähnen ermöglicht die freie Beweglichkeit beider Bögen gegeneinander (KaVo Dental GmbH 2008). 
Der paraokklusale Löffel besteht aus einer Metallauflage zur Aufnahme des SenderBogens und einem verformbaren Metallrand, welcher an die Unterkieferzähne der Patienten angepasst werden kann (siehe Abbildung 13). Der angepasste Löffel kann entweder direkt in der gleichen Sitzung oder nach vorheriger Abformung indirekt im Dentallabor an den vestibulär-Flächen des Unterkiefers fixiert werden (Ahlers 2009). Im Folgenden sollen die beiden Techniken näher beschrieben werden. Beide Techniken wurden bereits in unterschiedlichen Studien zu den verschiedensten Fragestellungen eingesetzt (Ohlendorf et al. 2008; 2012; 2015; Bräunig B 2012; Edelhoff 2015; Dejak et al. 2018; Mage et al. 2019). Jedoch wurde der Einfluss beider Anlegetechniken auf die Reliabilität der Messungen noch nicht untersucht.

\subsection{Direkte Anlegetechnik}

Der Metallrand des Löffels wird an der Unterkieferzahnkontur von vestibulär angepasst, sodass eine intraorale Verklebung beider mit einem plastischen Befestigungsmaterial erfolgen kann. Um statische oder dynamische Störkontakte durch das Befestigungsmaterial zu verhindern, müssen die Oberkieferzähne abgedeckt werden, um sie vor Überschüssen zu schützen. Die detaillierte Beschreibung der praktischen Durchführung ist unter Abschnitt 2.4.2 zu finden.

\subsection{Indirekte Anlegetechnik}

Hierfür werden zur Erstellung von benötigten Gipsmodellen Oberkiefer und Unterkiefer des Patienten abgeformt. Diese werden im zahntechnischen Labor verwendet, um im zweiten Schritt den sogenannten „Montagebehelf“ herstellen zu können. Auch hier wird der Metallrand des Löffels an der Unterkieferzahnkontur angebogen. Dafür müssen die Modelle in statischer Okklusion fixiert werden. Der Zahntechniker beschichtet den Metallrand des Löffels mit Kunststoff und adaptiert ihn an den Vestibulärflächen des Unterkiefers. Nach der Polymerisation wird der Kunststoff auf eine Dimension getrimmt, die eine ungestörte statische oder dynamische Okklusion zulässt. Der so individualisierte Löffel wird dementsprechend - mit Ausnahme der Abformung - hauptsächlich im Labor und unter zusätzlichem Zeit- und Kostenaufwand hergestellt (Ahlers 2009). Eine detaillierte Beschreibung der Vorbereitung und Durchführung ist in den Kapiteln 2.3.3 und 2.4.3 zu finden. 


\section{$1.2 \quad$ Fragestellung}

Ziel der vorliegenden klinischen Studie war die Untersuchung des Einflusses der Anlegetechnik des paraokklusalen Löffels, des Geschlechts und das Vorliegen eines eventuellen Tiefbisses auf die Reliabilität elektronischer Registriersysteme am Beispiel des ARCUSdigma II. Hierzu wurden funktionsgesunde ProbandInnen rekrutiert und der Kondylenbahnneigungswinkel sowie der Bennett-Winkel mittels direkter und indirekter Anlegetechnik in drei Sitzungen im Abstand von zwei Wochen gemessen. Im Anschluss wurden die Daten statistisch ausgewertet, um den Einflusses der Anlegetechnik, des Geschlechts und eines eventuellen Tiefbisses zu bestimmen. Bisher gab es keine evidenzbasierte, klinische Empfehlung zur Anlegetechnik des paraokklusalen Löffels sowie möglicher modifizierender, patientenindividueller Faktoren. Daher zielt diese Untersuchung darauf ab, beide Anlegetechniken hinsichtlich ihrer Reliabilität zu untersuchen und den Einfluss des Geschlechts und eines eventuell vorliegenden Tiefbisses zu ermitteln. 


\section{Material und Methoden}

\subsection{Genehmigung der klinischen Studie}

Die vorliegende Studie (Antrag Nr. 23/3/14) wurde von der Ethikkommission der Medizinischen Fakultät der Georg-August-Universität Göttingen genehmigt. Alle ProbandInnen wurden detailliert über die Studie aufgeklärt und willigten schriftlich in die Studienteilnahme ein. Die praktische Untersuchung wurde an der Poliklinik für Zahnärztliche Prothetik in Göttingen (Direktor: Prof. Dr. R. Bürgers) im Zeitraum von Juni 2014 bis einschließlich Februar 2015 durchgeführt. Eine Übersicht zum Studiendesign ist in Abbildung 14 dargestellt.

\subsection{ProbandInnen und Ausschlußkriterien}

Rekrutiert wurden insgesamt 14 funktionsgesunde Probandinnen und Probanden mit einem durchschnittlichen Alter von 24,9 Jahren ( \pm 2,3 Jahre). An der Studie nahmen elf weibliche und drei männliche ProbandInnen teil. Es sollten nur ProbandInnen teilnehmen, die mindestens 18 Jahre alt waren und keine Funktionsstörungen des Kauorgans, d. h. Craniomandibulären Dysfunktionen (CMD), aufwiesen (,funktionsgesund“). Des Weiteren sollten alle TeilnehmerInnen eine vollständige Dentition mit Klasse-IVerzahnung aufweisen. Ausschlusskriterien waren festsitzender oder herausnehmbarer Zahnersatz, festsitzende kieferorthopädische Apparaturen oder funktionseinschränkende Okklusionsbefunde (Nonokklusion, Hyperbalancen, frontal offener Biss, lateraler Kreuzbiss, Kopfbiss, Scherenbiss sowie Angle-Klasse II und III).

\subsubsection{Ausschluss von CMD}

Hierzu erfolgte das funktionelle Screening nach Ahlers (DentaConcept Verlag, Hamburg, Deutschland). Überprüft wurden folgende Kriterien: Bewertung der Mundöffnung (gerade/asymmetrisch/eingeschränkt), Auskultation von Gelenkgeräuschen (keine/Reiben/Knacken) und okklusale Geräusche (tiefer Ton/hoher Ton) sowie die Muskelpalpation (unauffällig/druckdolent) und die Erfassung traumatischer, exzentrischer Schlifffacetten. Der Ausschluss aus der Studie erfolgte bei einem Score von zwei oder mehr Auffälligkeiten. 


\subsubsection{Dentaler Befund}

Alle Teilnehmer erhielten eine zahnärztliche Voruntersuchung von einem erfahrenen Oberarzt der Poliklinik für Zahnärztliche Prothetik (Dr. H. Rasing), um mögliche dentale Störfaktoren auszuschließen. Der dentale Befund umfasste die Bewertung der statischen und dynamischen Okklusion, die Untersuchung der Vitalität und den Ausschluss von Perkussionsempfindlichkeiten der Zähne. Ein besonderes Augenmerk lag auf der Messung des sagittalen und vertikalen Überbisses. Funktionseinschränkende Funktionsbefunde (siehe Kapitel 2.2) führten zum Ausschluss aus der Studie.

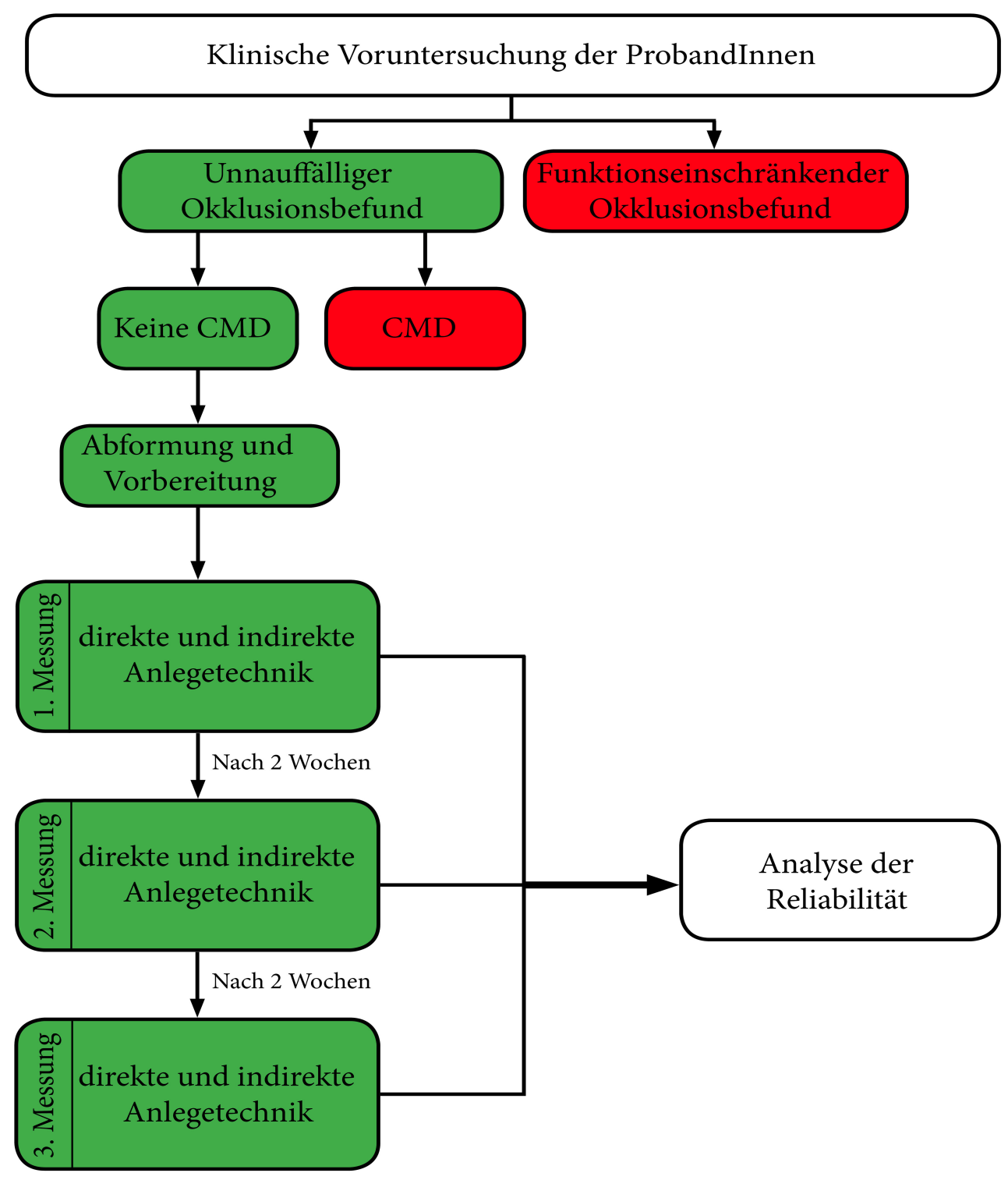

Abbildung 14: Studiendesign mit Ausschlusskriterien (rot) 


\subsection{Vorbereitung}

Nach vollständiger Aufklärung der ProbandInnen, deren Einwilligung in die Studienteilnahme und Überprüfung der Ausschlusskriterien wurden die Oberkiefer- und Unterkieferzahnreihen abgeformt, um im zahntechnischen Labor Situationsmodelle der jeweiligen ProbandInnen herzustellen. Die Situationsmodelle wurden benötigt, um die individualisierten, paraokklusalen Löffel anzufertigen.

\subsubsection{Abformung}

In der ersten Sitzung wurden bei allen ProbandInnen Alginat-Abformungen (Palgat ${ }^{\mathrm{TM}}$ Plus, 3M Espe, Seefeld, Deutschland) vom Oberkiefer und Unterkiefer angefertigt. Zudem wurde ein Bissregistrat in habitueller Okklusion aus einem extraharten, schnellabbindenden A-Silikon (Registrado X-tra, VOCO GmbH, Cuxhaven, Deutschland) erstellt, um die Modelle im Labor einartikulieren zu können.

\subsubsection{Modellherstellung}

Als nächstes wurden für alle ProbandInnen jeweils ein Oberkiefer- und ein UnterkieferSituationsmodell aus Superhartgips (Picodent quadro-rock ${ }^{\circledR}$, picodent ${ }^{\circledR}$ DentalProduktions- und Vertriebs-GmbH, Wipperfürth, Deutschland) hergestellt. Im Anschluss konnten die Modelle in habitueller Okklusion (d.h. in habitueller Bisslage des Probanden) mithilfe des Bissregistrates manuell zusammengesetzt werden.

\subsubsection{Individualisierung der paraokklusalen Löffel}

Paraokklusale Löffel dienten der Fixierung des Senderbogens der digitalen Funktionsanalyse am Unterkiefer, um damit die Funktionsbewegungen des Unterkiefers zum Oberkiefer aufzuzeichnen. Die paraokklusalen Löffel (Referenznummer 1.000.9291, KaVo Dental GmbH, Biberach, Deutschland) wurden mit lichtpolymerisierendem Kunststoff (Impression Tray Resin LC, Henry Schein Dental Deutschland GmbH, Langen, Deutschland) an den Unterkiefermodellen adaptiert und ermöglichten so die spätere Fixierung des Löffels an den Unterkiefern der ProbandInnen. Das Einbringen einer Wachsplatte (HS-Modellierwachs Universal, Henry Schein Dental Deutschland GmbH, Langen, Deutschland) zwischen Ober- und Unterkiefer und somit die Trennung der vestibulären Flächen der Oberkieferzähne von den Unterkieferzähnen beim Individualisieren der paraokklusalen Löffel erleichterte die Gestaltung und verhinderte eine Überdimensionierung des jeweiligen individuellen Löffels, sodass keine 
Störkontakte in der statischen und dynamischen Okklusion entstehen konnten (siehe Abbildung 15). Zum Aushärten des Kunststoffes wurden die Unterkiefermodelle mit den anmodellierten paraokklusalen Löffeln in einem Lichtofen (LUXOMAT D, al dente Dentalprodukte GmbH, Horgenzell, Deutschland) für 10 Minuten ausgehärtet. Nach dem Aushärten des Kunststoffes wurden die Löffel von den Unterkiefermodellen gelöst und auf scharfe Kanten bei Bedarf mit einer Hartmetallfräse und Sandpapier geglättet.

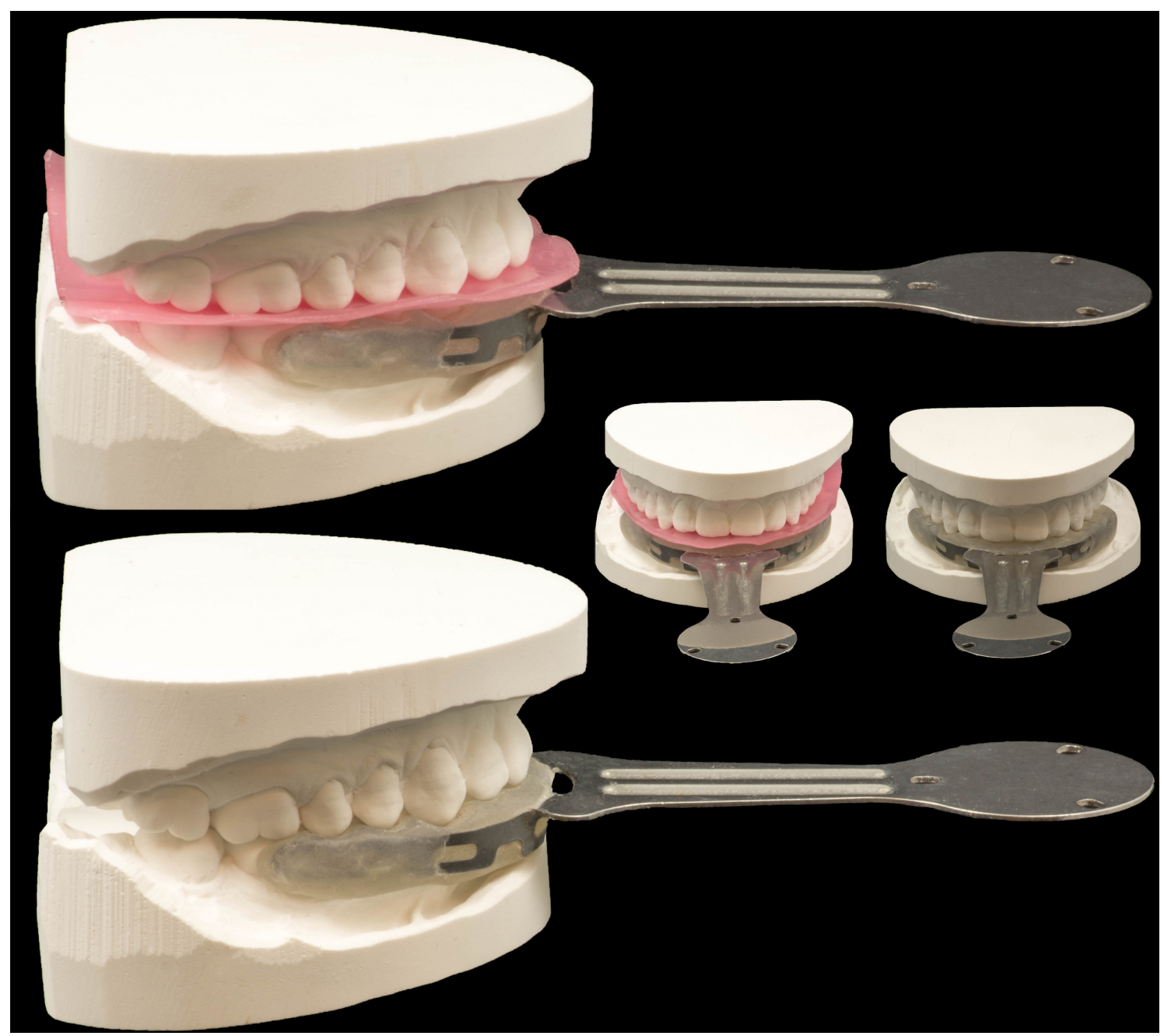

Abbildung 15:Individualisierung der paraokklusalen Löffel mit lichtpolymerisierendem Kunststoff und Anpassen an die Gipsmodelle der ProbandInnen

\subsection{Messungen}

Alle 14 ProbandInnen wurden in drei Zyklen zur Vermessung mit dem ARCUSdigma II (KaVo Dental GmbH, Biberach, Deutschland) einbestellt (siehe Abbildung 14). Um den Einfluss der Anlegetechnik auf die Reliabilität der Ergebnisse zu untersuchen, wurde bei 
jedem Probanden sowohl die direkte als auch die indirekte Befestigung der paraoklusalen Löffel am Unterkiefer durchgeführt. Die Messungen fanden mit einem zeitlichen Abstand von zwei Wochen in derselben zahnärztlichen Behandlungseinheit der Poliklinik für Zahnärztliche Prothetik der Universitätsmedizin Göttingen statt. Bei jeder der drei Messungen wurden Bennett-Winkel (BA) und Kondylenbahnneigungswinkel (HCI) des jeweiligen ProbandIn unter Anwendung des ARCUSdigma II Messprogramms „Artikulatorprogrammierung" (KaVo Dental GmbH, Biberach, Deutschland) ermittelt.

\subsubsection{Anlegen des ARCUSdigma II}

Das Anlegen des ARCUSdigma II-Registriersystems erfolgte entsprechend der Anleitung des Herstellers. Hierbei wurden die ProbandInnen in eine aufrechte Sitzposition gebracht. Der Empfängerbogen wurde mit Hilfe der Nasenstütze an der Nasenwurzel sowie mit den Ohroliven des Befestigungsbogens im äußeren Gehörgang befestigt. Zusätzlich wurde der Bogen mit einem Gummiband im Bereich des Hinterhaupts fixiert. Somit konnte eine stabile und komfortable Fixierung über die gesamte Messdauer gewährleistet werden (siehe Abbildung 16). Die Frankfurter Horizontale diente beim Anlegen zur Orientierung (siehe Abbildung 9). Um reproduzierbare posteriore Referenzpunkte für die Artikulatorprogrammierung zu definieren, wurde stets das KaVo-Transfer-System (KTS) (KaVo Dental GmbH, Biberach, Deutschland) für die Kalibrierung vor jeder Messung verwendet. Unter Anwendung der KTS-Bissgabel (Referenznummer 1.000.8518, KaVo Dental GmbH, Biberach, Deutschland) und dem Senderbogen wurde bei jedem ProbandIn zunächst die dreidimensionale Lagebeziehung zwischen Maxilla und Empfängerbogen registriert. Hierzu wurden die Bissgabel mit Registrado (Registrado X-tra, VOCO GmbH, Cuxhaven, Deutschland), einem additionsvernetzenden RegistrierSilikon, beschichtet und auf den Okklusalenflächen der Oberkieferzähnen fixiert. Nach der Abbindephase von 40 Sekunden waren die Impressionen der Zähne im Registriermaterial gesichert (siehe Abbildung 17). Die so präparierte KTS-Bissgabel konnte nun für alle nachfolgenden Messungen des jeweiligen ProbandIn reproduzierbar am Oberkiefer angebracht werden. Mit Hilfe dieses Verfahrens sollten Abweichungen im Referenzachsenprocedere ausgeschlossen werden. 


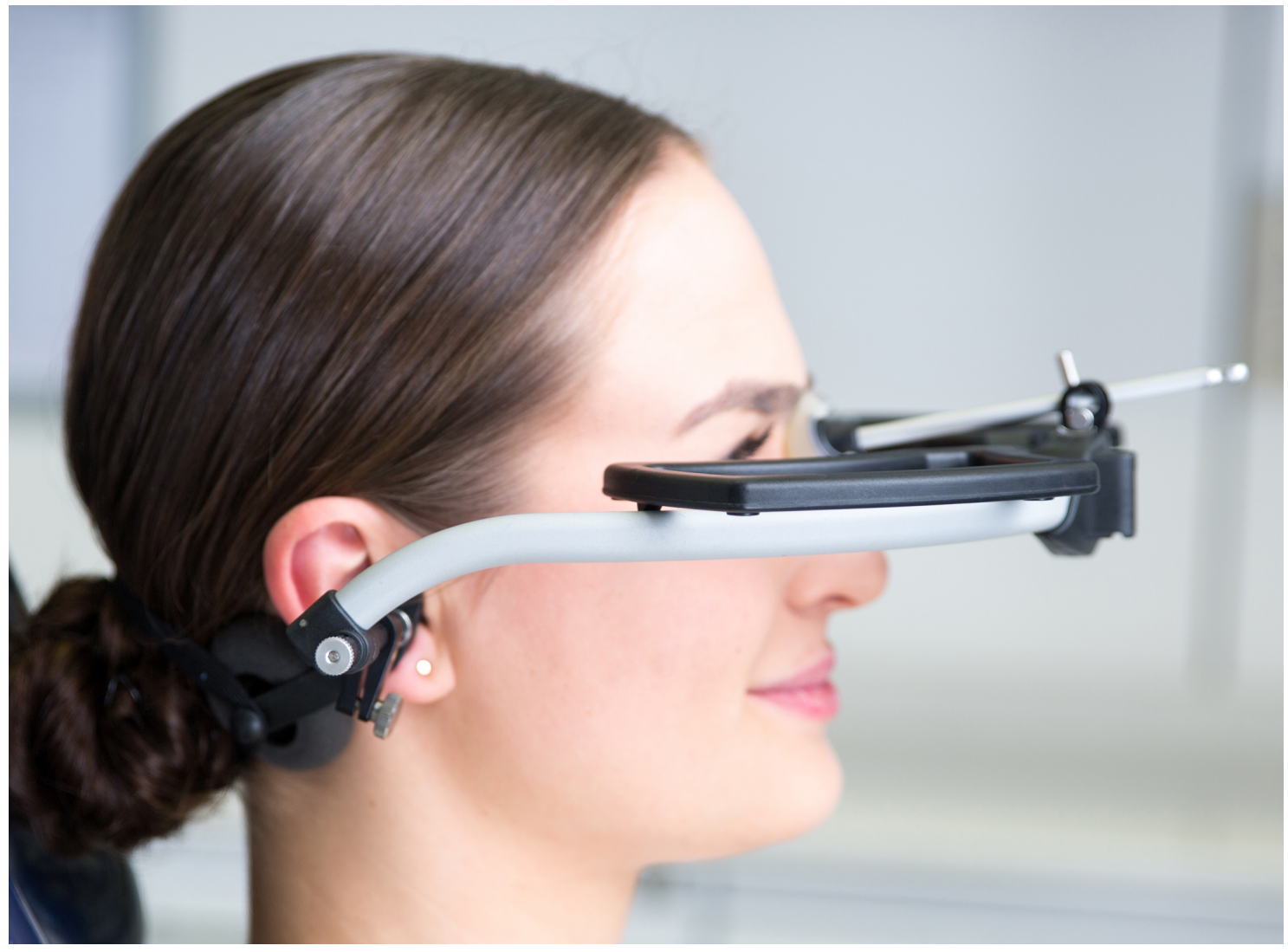

Abbildung 16: Angelegter Empfängerbogen

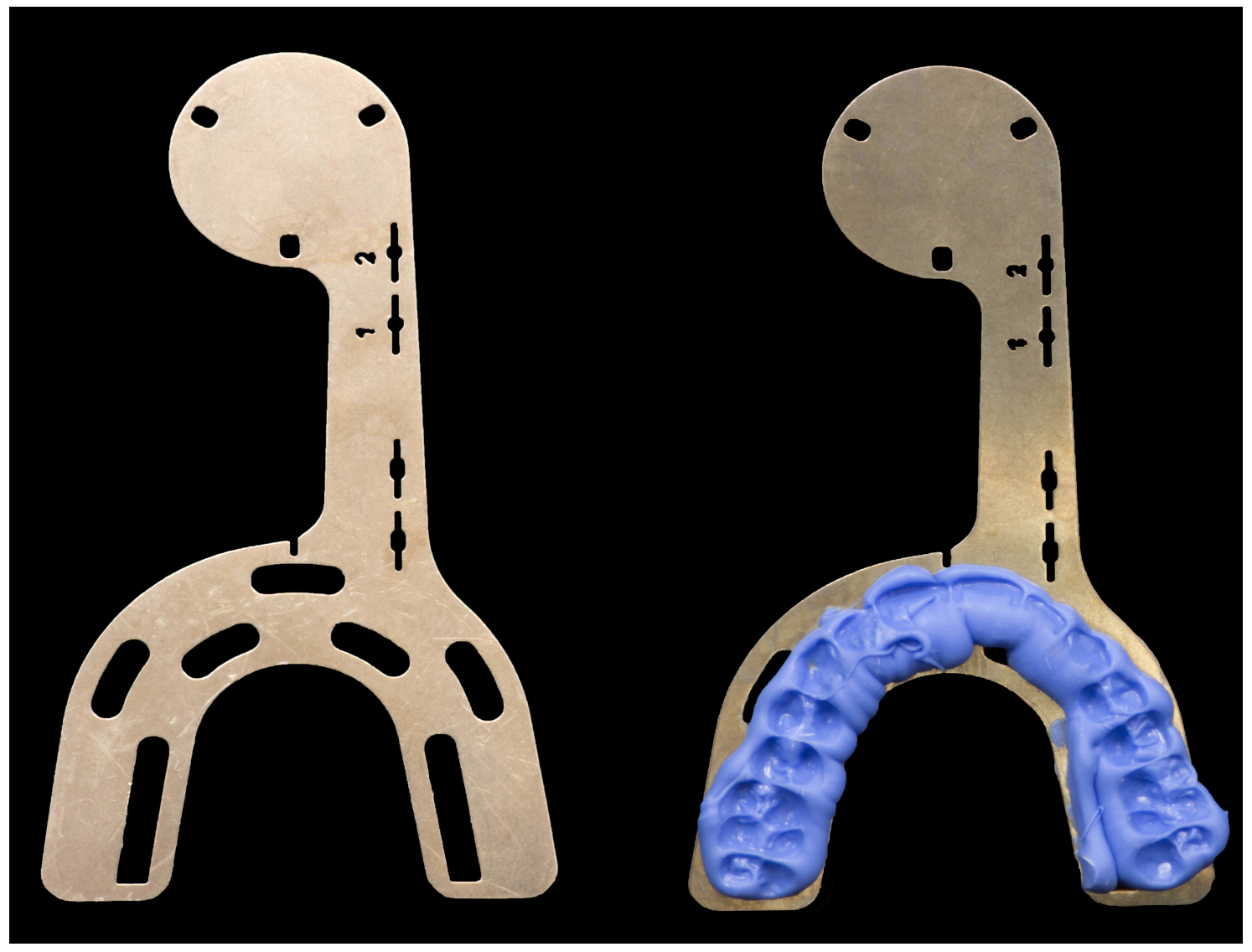

Abbildung 17: Oberkiefer-Bissgabel mit und ohne Individualisierung 
Anschließend wurde die Lage der Mandibula bei maximaler Interkuspidation mit dem Senderbogen und dem paraokklusalen Löffel registriert. Dafür wurde der Senderbogen jeweils nach der direkten sowie der indirekten Anlegetechnik fixiert und die Messung gestartet.

\subsubsection{Direkte Anlegetechnik}

Bei der direkten Anlegetechnik wurde der konfektionierte paraokklusale Löffel zunächst mit einer Flachspitzzange (Artikelnummer 363008, Henry Schein Dental Deutschland $\mathrm{GmbH}$, Langen, Deutschland) an die Frontzahnkontur adaptiert (siehe Abbildung 18). Als weitere vorbereitende Maßnahme wurden die ProbandInnen gebeten, analog zur Vorbereitung der indirekten Anlegetechnik (siehe Kapitel 2.3.3 Individualisierung der paraokklusalen Löffel), in weiches Basisplattenwachs (HS-Modellierwachs Universal, Henry Schein Dental Deutschland GmbH, Langen, Deutschland) zu beißen (siehe Abbildung 19). Mit Hilfe dieser Wachsplatte konnten statische oder dynamische Störkontakte durch Überschüsse des Befestigungsmaterials vermieden werden.

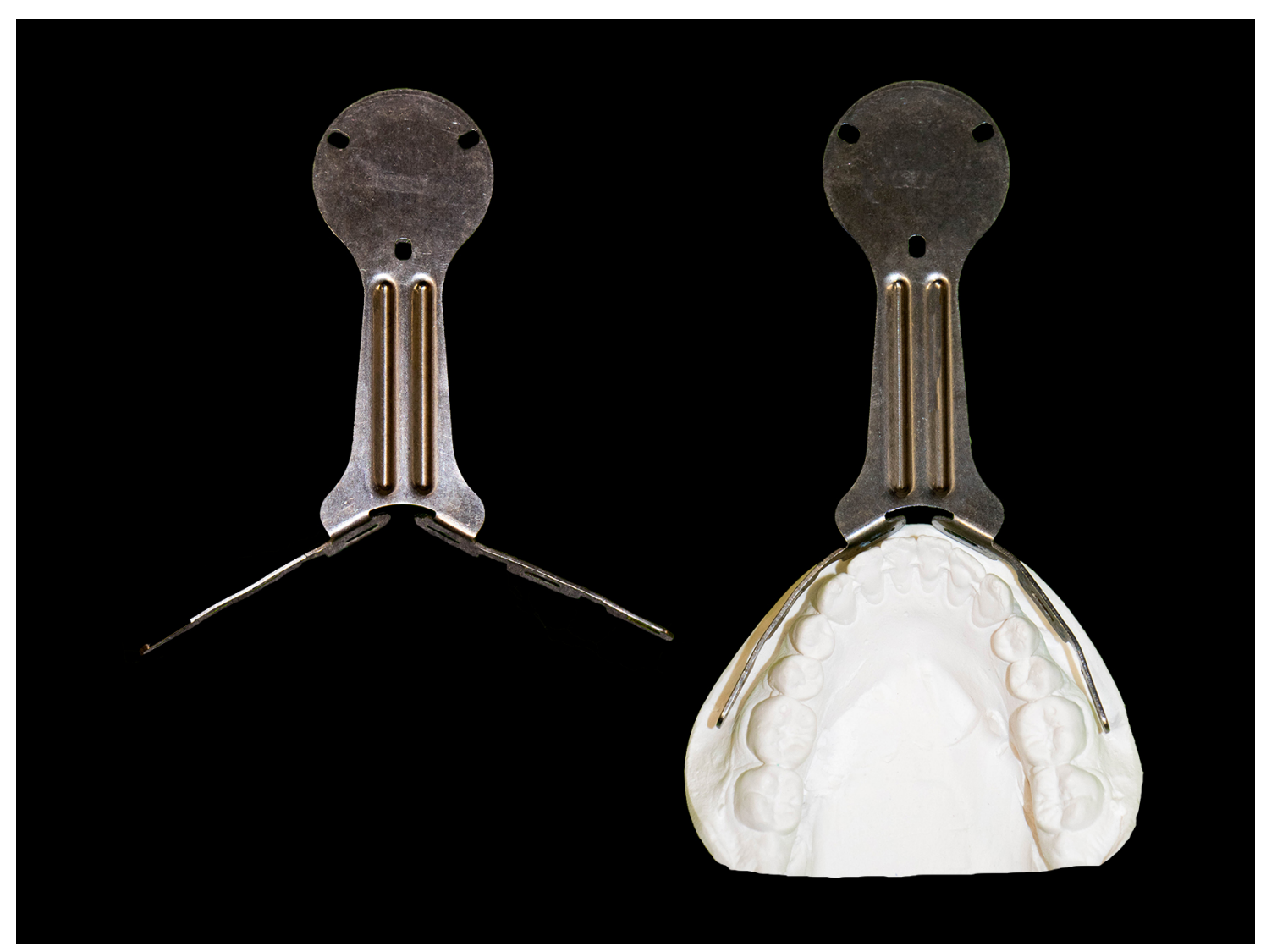

Abbildung 18: Ein konfektionierter, paraokklusaler Löffelvor und nach Adaptieren an die Frontzahnkontur (schematisch durch Gipsmodell dargestellt) 


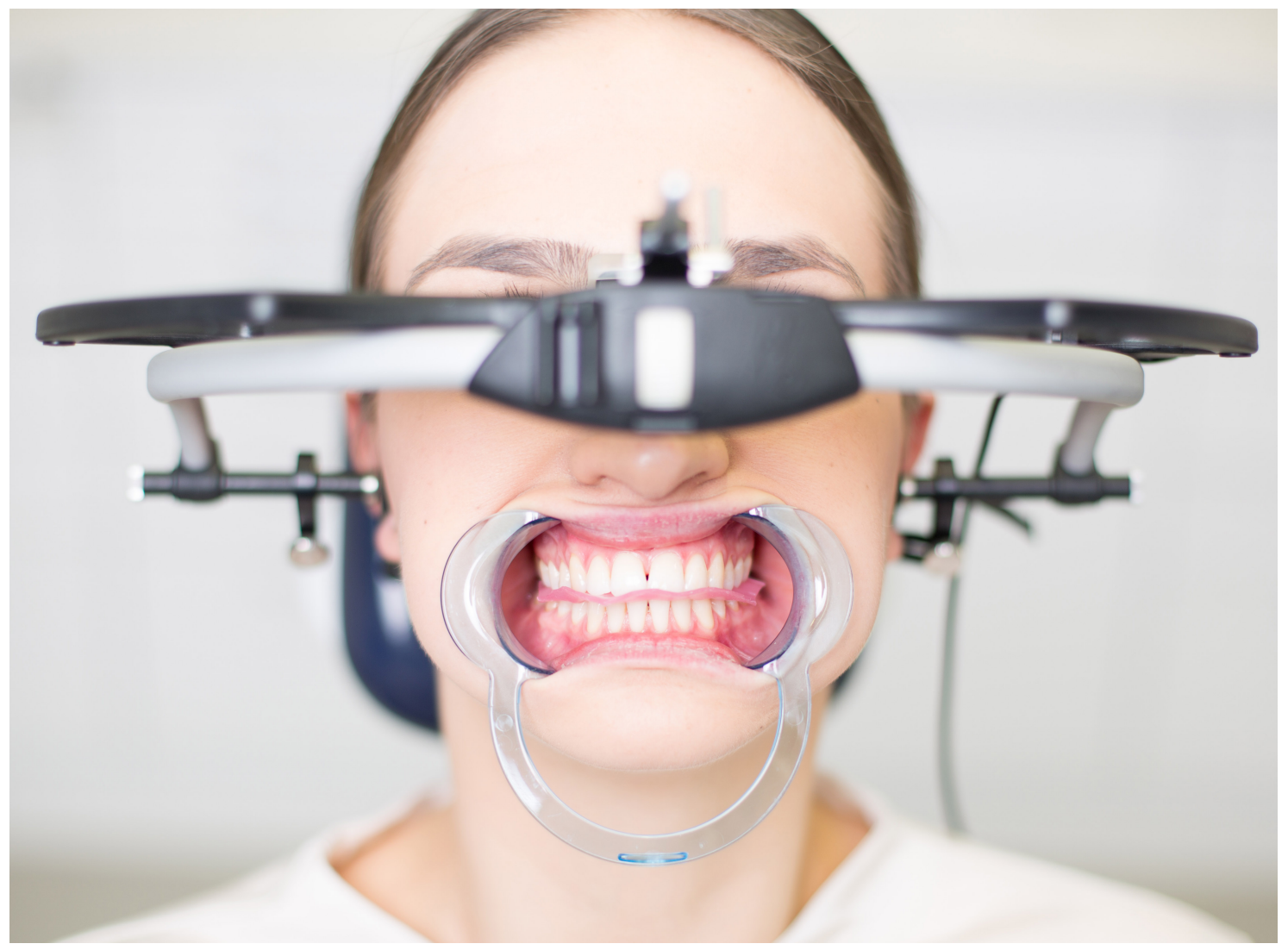

Abbildung 19: Einsetzen des Basisplattenwachses zum Vermeiden von Störkontakten

Anschließend wurde der Löffel im chairside-Verfahren, in Anlehnung an das Vorgehen von Ahlers, mit Registriermaterial (Registrado X-tra, VOCO GmbH, Cuxhaven, Deutschland) an den Labialflächen der unteren Front- und Seitenzähne befestigt (siehe Abbildung 20).

Somit konnte durch das Zusammenbeißen der Probanden eine klare Trennung der vestibulären Flächen der Oberkieferzähne von den Unterkieferzähnen gesichert werden. Nach der Abbindephase von 40 Sekunden konnte die Wachsplatte entfernt werden. Anschließend wurde der Senderbogen auf den fixierten Löffel aufgebracht und die Messung gestartet (siehe Abbildung 21). 


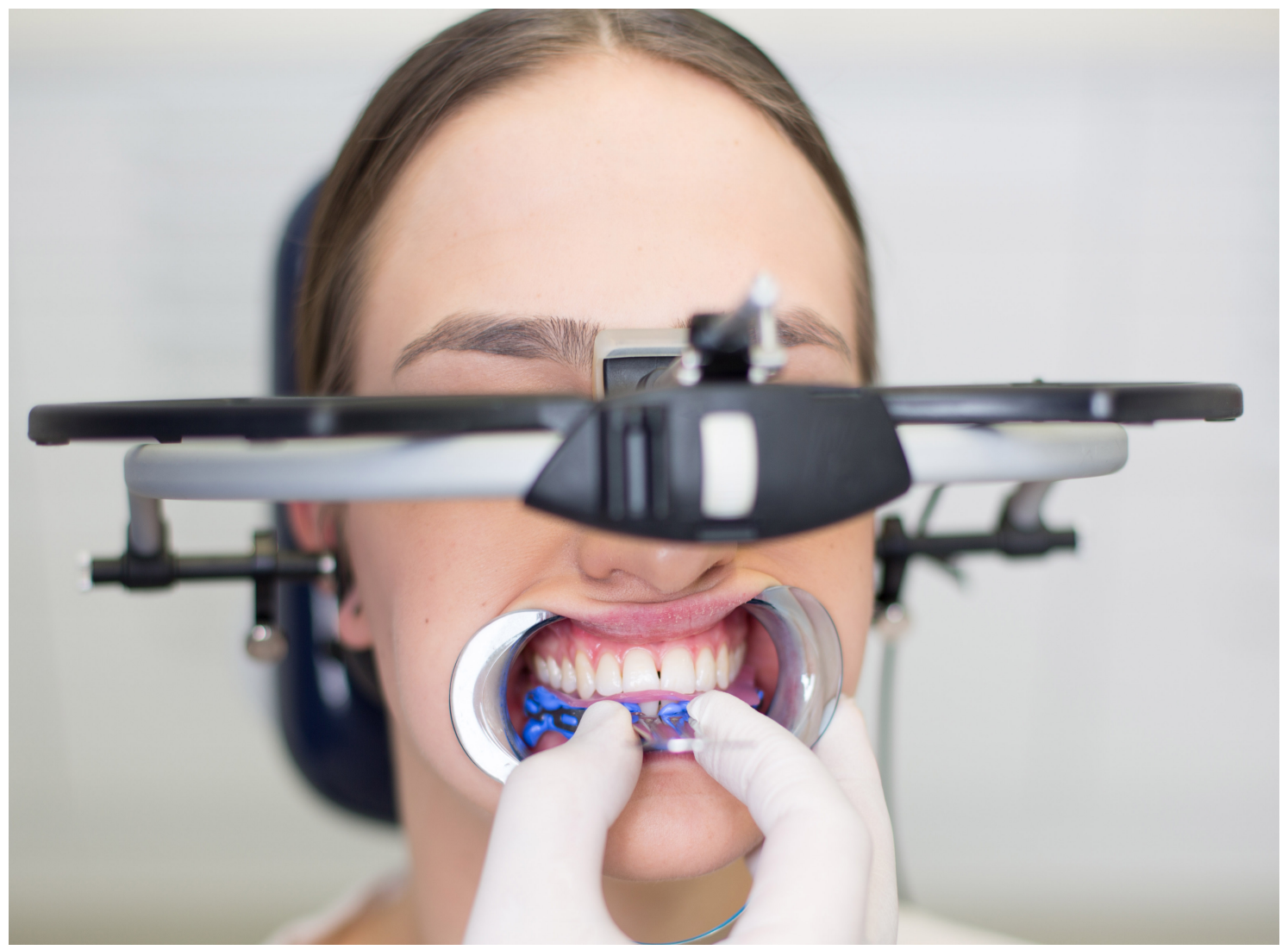

Abbildung 20: Adaption des paraokklusalen Löffels an die Unterkieferzähne

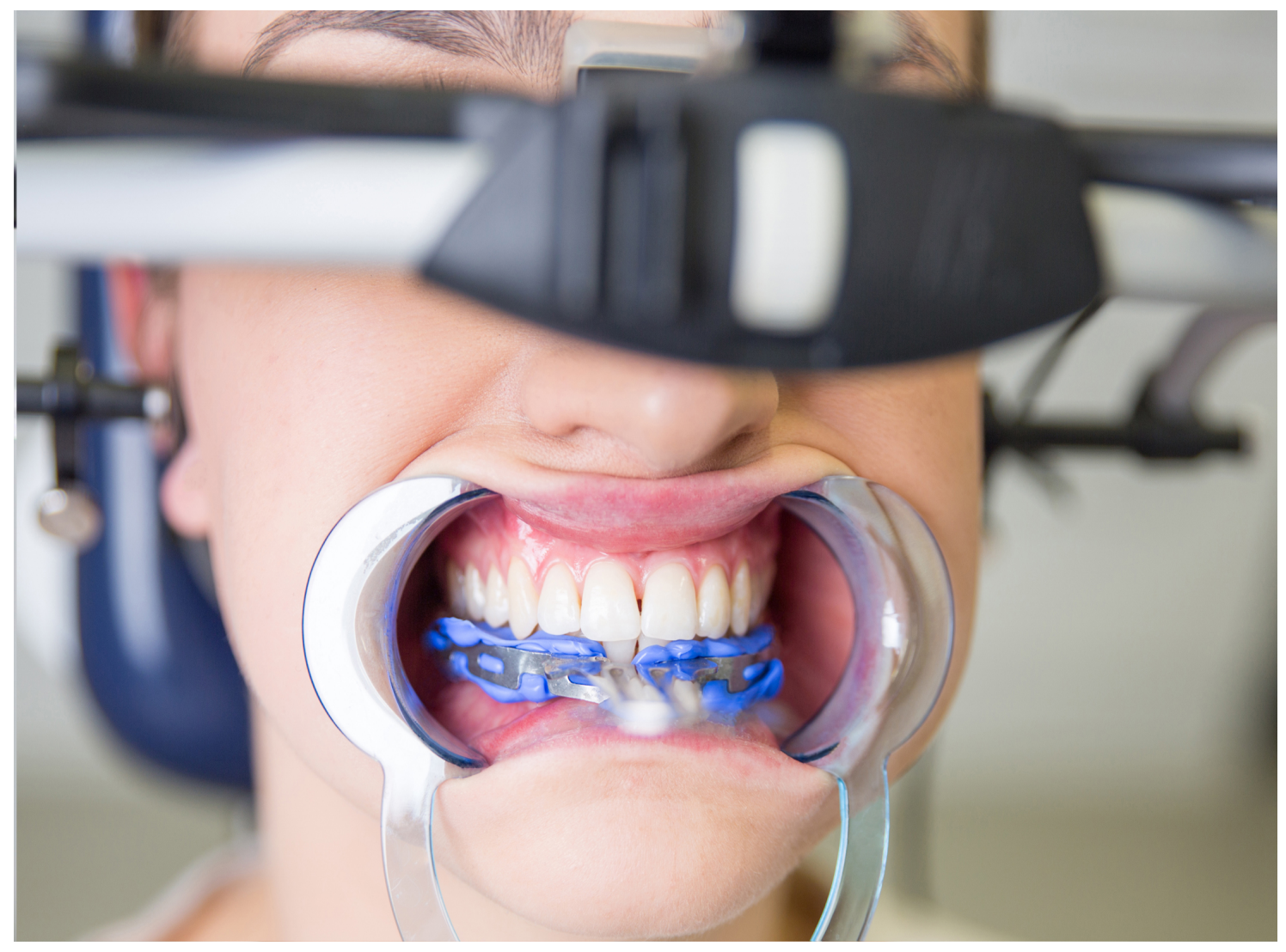

Abbildung 21: Endsituation nach der direkten Anlegetechnik (chairside) 


\subsubsection{Indirekte Anlegetechnik}

Die im Labor hergestellten paraokklusalen Löffel wurden im Unterkiefer ohne weitere Hilfsmittel befestigt, d .h. aufgesteckt (siehe Abbildung 22). Analog zum Vorgehen bei der direkten Anlegetechnik musste auch hier sichergestellt werden, dass sowohl die maximale Interkuspidation als auch dynamische, zahngeführte Bewegungen gleithindernisfrei eingenommen werden konnten.

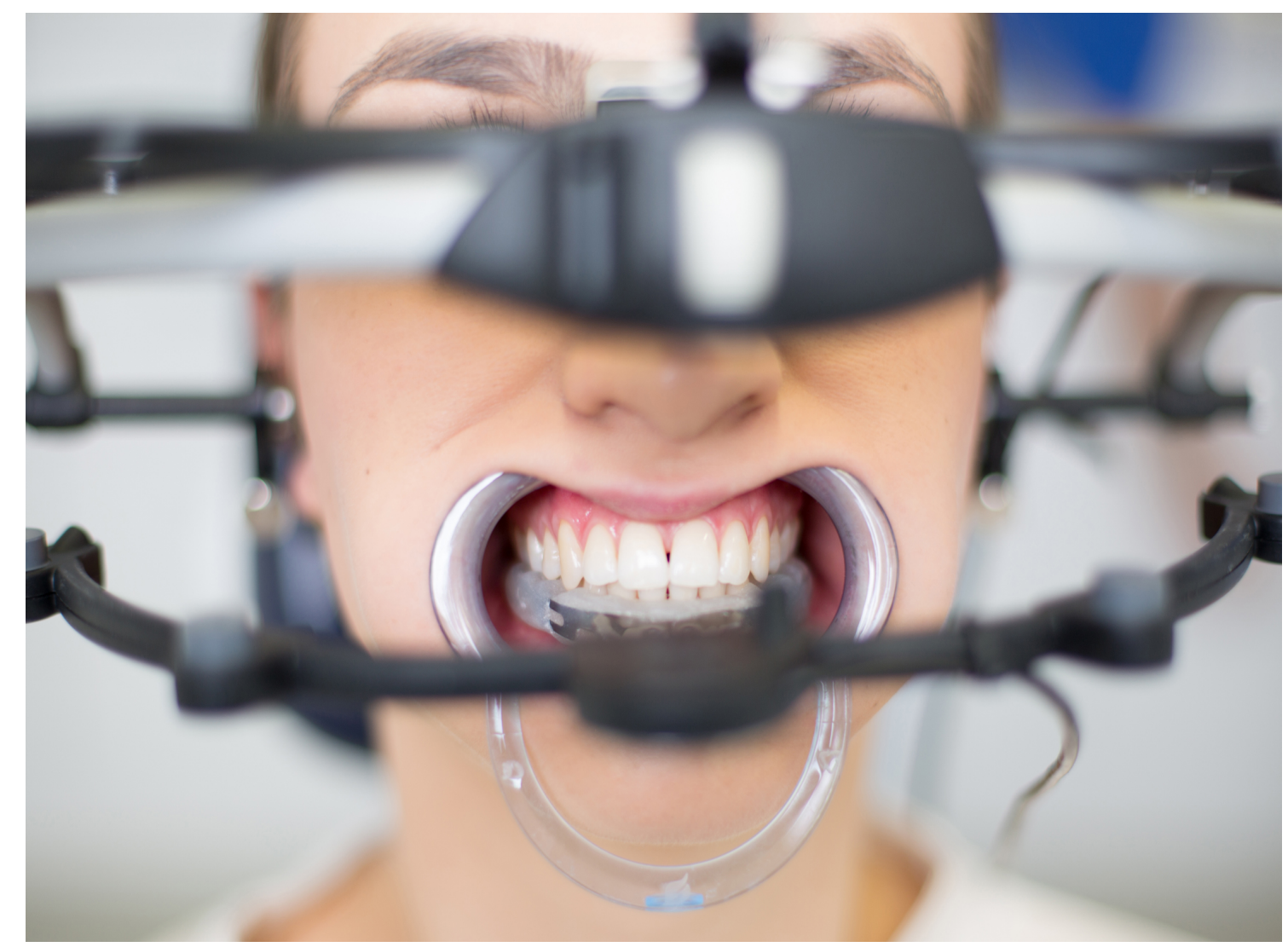

Abbildung 22: Abschlussbefund der indirekten Anlegetechnik mit individualisiertem paraokklusalen Löffel

\subsection{Vorbereitung der Messung}

Für diese Studie wurden alle Messungen auf einer Secure Digital Memory Card (SD-Chipkarte) (TS2GSDHC2 SD-Karte 2 GB, Transcend Information, Inc., Taipeh, Taiwan) gespeichert. Über den Touchscreen des Basisgeräts des ARCUSdigma IIRegistriersystems wurden die ProbandInnenstammdaten verschlüsselt angelegt und standen dann für die weiteren Messungen zur Verfügung. Um die verschlüsselten Daten zu speichern und um spätere Verwechslungen zu vermeiden, wurde jedem ProbandIn ein eindeutiger Identifizierungscode zugeteilt. 


\subsubsection{Kalibrierung des ARCUSdigma II}

Vor Beginn der Messung erfolgte bei beiden Anlegetechniken zunächst die Bestimmung der Oberkieferlage durch die Positionierung der OK-Bissgabel (Impressionen aus Registrado) an den Kauflächen der Oberkieferzähne der ProbandInnen. Die Bissgabel diente dabei der Befestigung des ARCUSdigma II Senderbogens. Der magnetische Senderbogen wurde - gemäß der Bohrungen - an der Bissgabelspitze arretiert. Der Fußschalter wurde betätigt, um die Lagebestimmung des Oberkiefers durchzuführen und das Gerät zu kalibrieren. Ein Tonsignal und die Anzeige des Bildschirms bestätigten die erfolgreiche Kalibrierung. Anschließend wurde die Oberkiefer-Bissgabel aus dem Mund des ProbandIn entfernt und der Senderbogen von der Bissgabel gelöst.

\subsubsection{Ablauf der Messungen}

$\mathrm{Zu}$ Beginn der Messungen wurde der Senderbogen mit Hilfe der magnetischen Arretierung auf dem, im Unterkiefer bereits fixierten, paraoklusalen Löffel angebracht. Für die Erfassung der Artikulatoreinstellwerte mussten die ProbandInnen den Unterkiefer in die maximale Protrusion sowie maximale linke und rechte Laterotrusion bewegen. Die ProbandInnen wurden dann aufgefordert, in die maximale Interkuspidation (IKP) zu gehen. Dann wurde der Fußschalter getätigt und gehalten, während der/die ProbandIn unter Zahnführung maximal protrudierte. Der Fußschalter wurde gelöst, wenn die maximale Protrusion erreicht wurde. Aus der wieder eingenommenen IKP wurden die maximale Laterotrusion nach links und rechts erfasst (siehe Abbildung 23). Um eine präzise Analyse der Kondylenbahnneigung und des Bennett-Winkels zu erhalten, wurden diese Bewegungsaufzeichnungen dreimal wiederholt. Das System erstellte dann automatisch, nachdem alle Messungen durchgeführt wurden, einen Report aller Artikulatoreinstellwerte. Dieser wurde als Portable Document Format (PDF-Datei) exportiert und ausgedruckt (siehe Abbildung 24) 


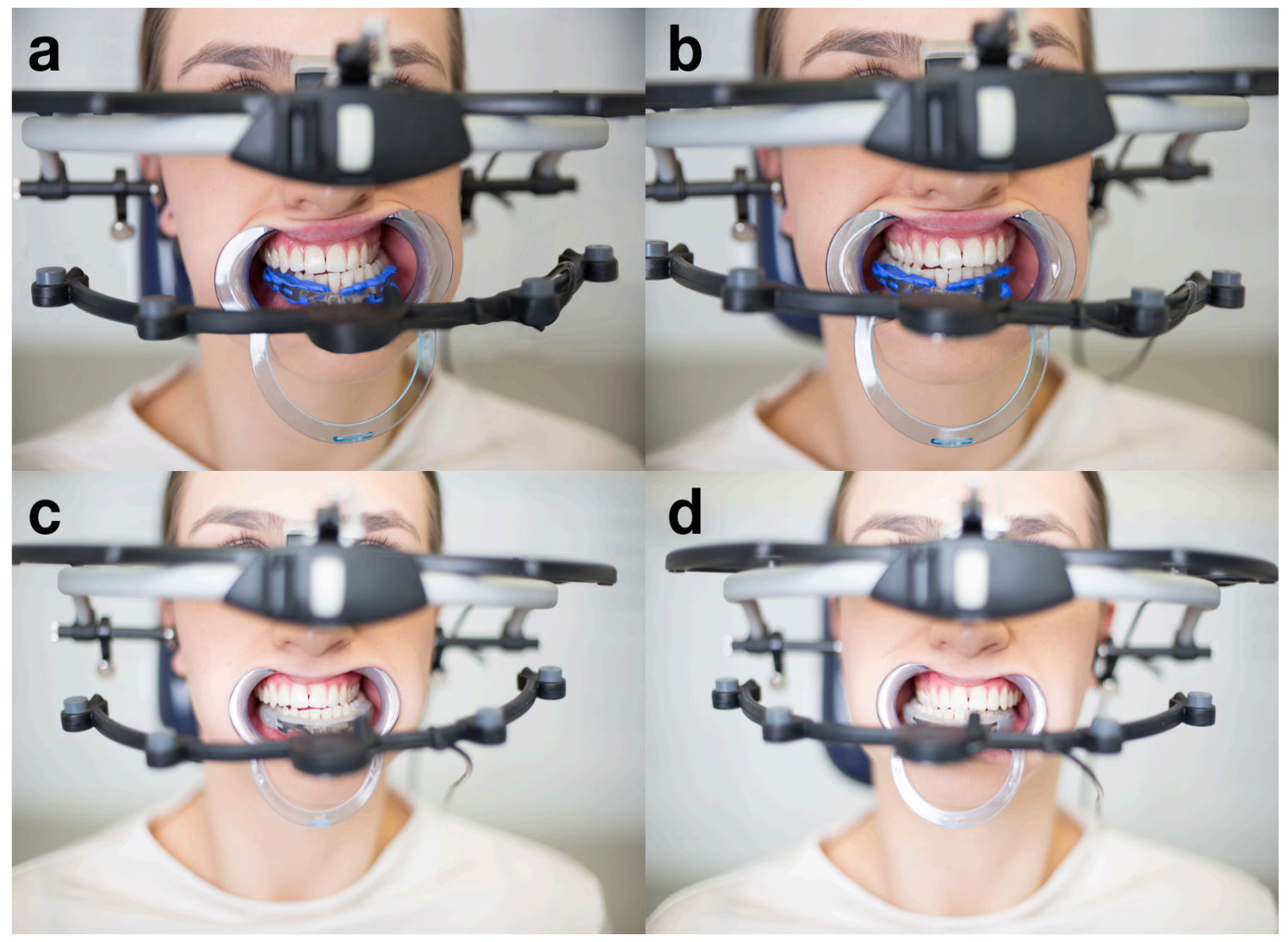

Abbildung 23: Während der Messung durchgeführte Unterkieferbewegungen: $\mathrm{a}=$ Laterotrusion links, $\mathrm{b}=$ Laterotrusion rechts (direktes Verfahren) und $\mathrm{c}=$ Laterotrusion links, $\mathrm{b}=$ Laterotrusion rechts (indirektes Verfahren)

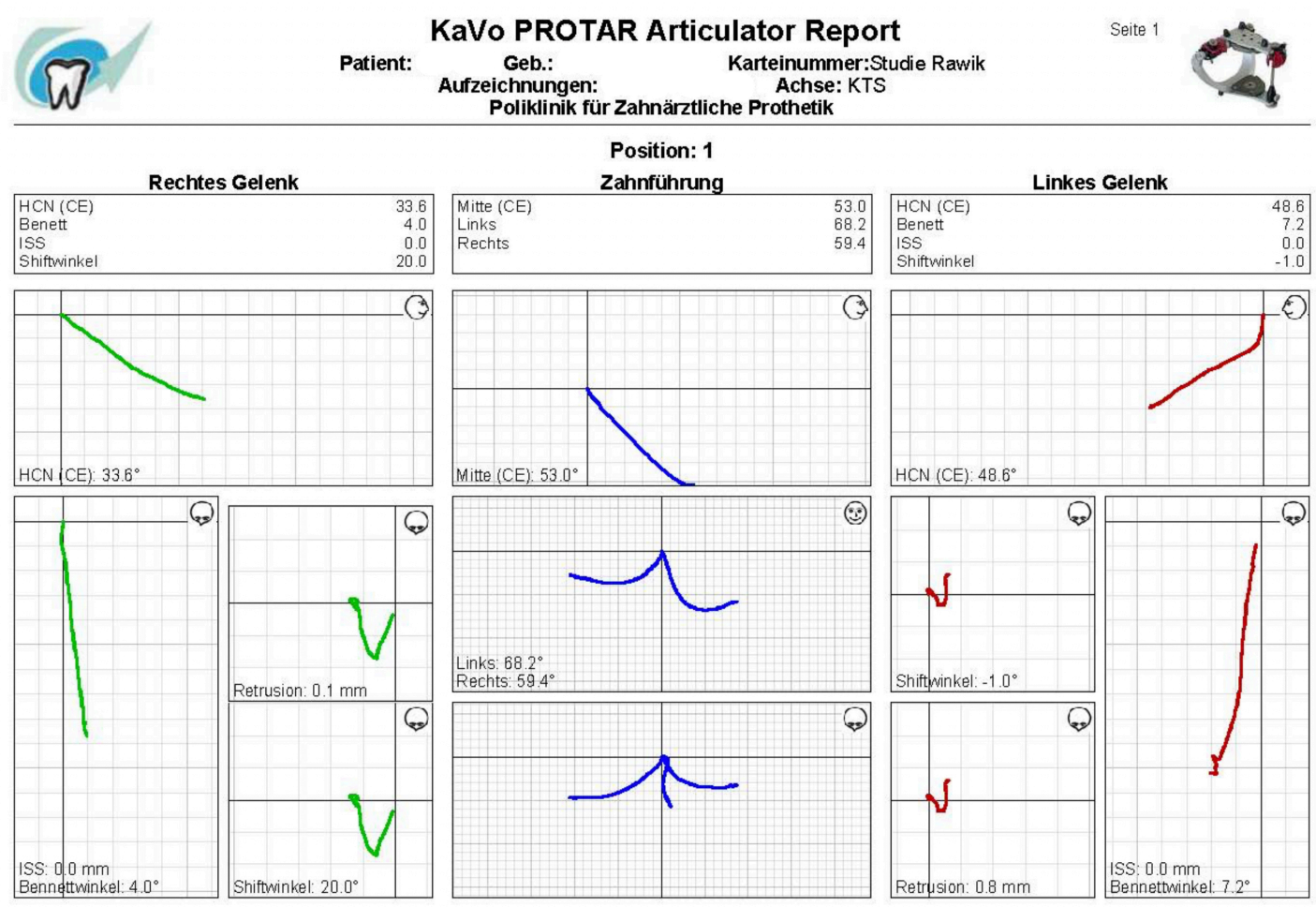

Abbildung 24: Artikulator-Report nach einer Messung 


\subsubsection{Messungsprocedere}

Das primäre Ziel der Studie war es, den Einfluss der Anlegetechnik auf die Reproduzierbarkeit der Messungen zu verschiedenen Zeitpunkten und die Reproduzierbarkeit der Messdaten per se zu untersuchen. Die Messungen wurden in einem zeitlichen Abstand von jeweils zwei Wochen erhoben (siehe Abbildung 25). Es wurde immer mit der direkten Anlegetechnik begonnen, die Messung der indirekten Anlegetechnik fand im Anschluss statt. Zwischen der direkten und der indirekten Messung wurde das ARCUSdigma II komplett abgenommen und wieder angelegt. So sind bei zwei Messtechniken und drei Messzyklen insgesamt sechs Messungen für jeden ProbandIn durchgeführt worden. Jede Messung lieferte die Artikulatoreinstellwerte für das rechte und das linke Kiefergelenk.

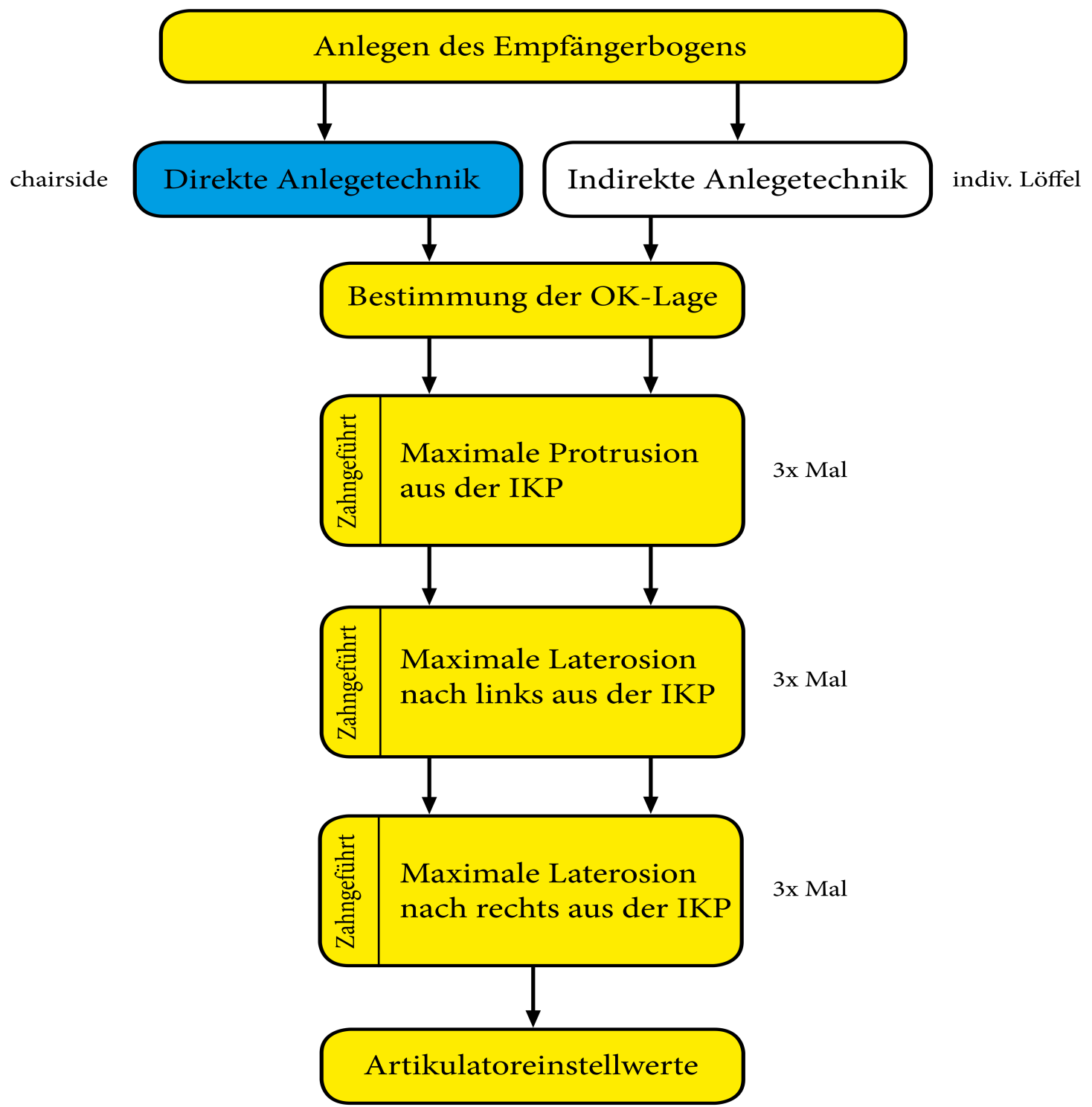

Abbildung 25: Übersicht des Messungsprotokolls 


\subsection{Statistik}

Die Daten der wiederholten Messungen wurden aus dem KaVo Integrated Desktop (KaVo Dental GmbH, Biberach, Deutschland) exportiert und in Microsoft Excel 2010 (Microsoft Corporation, Redmond, USA) zusammengeführt, um die absoluten Mittelwerte zu berechnen. Mit der Software XLSTAT (Addinsoft, Paris, Frankreich) erfolgte die Datenanalyse anhand deskriptiver Statistik und weiterführender statistischer Verfahren. Nach Durchführung des Shapiro-Wilk-Tests wurde eine Normalverteilung der Messungen bestätigt und somit waren die Voraussetzungen für die Anwendung parametrischer Testverfahren gegeben.

Die Genauigkeit der beiden Anlegetechniken im Hinblick auf die Messung von HCI und BA wurde mit Hilfe der Test-Retest-Methode für das jeweilige Messverfahren separat ermittelt. Aus den drei wiederholten Messungen des HCI bzw. des BA wurden zunächst für jedes Kiefergelenk die beiden Messungen mit der größten Inter-Session-Differenz ausgewählt. Die Übereinstimmungen der jeweiligen Werte wurden anschließend mit Hilfe des Pearson Korrelationskoeffizienten ( $r$ ) gemäß der folgenden Verteilung bestimmt: $r=0-0.29 / 0.30-0.49 / 0.50-0.69 / 0.70-0.99 / 1.00$ entsprechend keine/schwache/moderate/starke/perfekte lineare positive Relation.

Im Folgenden wurden die Abweichungen der individuellen Messungen sowie die Streuungen der durchschnittlichen Abweichungen im Hinblick auf ihre klinische Relevanz bewertet. Hierzu wurde die Schiefe der beiden Messmethoden mit Hilfe der Bland-Altman-Analyse quantifiziert und die Methoden auf Gleichwertigkeit überprüft. Anhand des Bland-Altman-Plots werden Differenzen der beiden Messmethoden gegen den Mittelwert der beiden Methoden grafisch aufgetragen, wobei der Mittelwert beider Messverfahren eine bestmögliche Schätzung eines unbekannten tatsächlichen Wertes darstellt. Die Abweichung zwischen beiden Methoden, die Ausreißer und die Schiefe der Ergebnisse ermöglichen Rückschlüsse auf Trends, wie zum Beispiel systematisch größere oder systematisch kleinere Werte einer jeweiligen Messmethodik. Die Übereinstimmungsgrenzen sind dabei durch die Formel $\mathrm{d} \pm 2 \times \mathrm{s}$ definiert, wobei $\mathrm{d}$ dem Mittelwert der Differenzen und s der Standardabweichung der Differenzen entspricht. Bei einer hinreichend symmetrischen Verteilung der Differenzen, liegen 95 \% der Werte im Bereich der Übereinstimmungsgrenzen. Die Bland-Altman Analyse wurde angewendet, um die Schwankungsbreite beider Anlegetechniken zu quantifizieren und die Methoden auf Gleichwertigkeit zu überprüfen. 
Mit der mehrfaktoriellen Varianzanalyse (mehrfaktorielle ANOVA, von analysis of variance) wurde überprüft, ob die Variabilität der Kondylenbahnwerte durch die Anlegetechnik, das Geschlecht oder den vertikalen Überbiss der ProbandInnen erklärt werden kann. Die gleiche Analyse wurde für den Bennett-Winkel durchgeführt. 


\section{Ergebnisse}

\subsection{Erläuterungen zu den Grafiken}

Die Messwerte und statistischen Ergebnisse der vorliegenden Arbeit sind zum Teil in Whisker-Box-Plots dargestellt. Das Zentrum von Whisker-Box-Plots ist die Box, die durch den Median geteilt wird und $50 \%$ der Messwerte beinhaltet. Die obere und untere Grenze der Box wird durch das untere Quartil und das obere Quartil gebildet. Werte außerhalb der Box werden durch Linien, die sog. Whisker dargestellt. Diese sind auf die eineinhalbfache Länge der Box begrenzt, sodass Messwerte außerhalb dieses Rahmens durch Punkte als Ausreißer präsentiert werden. Abbildung 26 zeigt exemplarisch den Aufbau eines Whisker-Box-Plots.

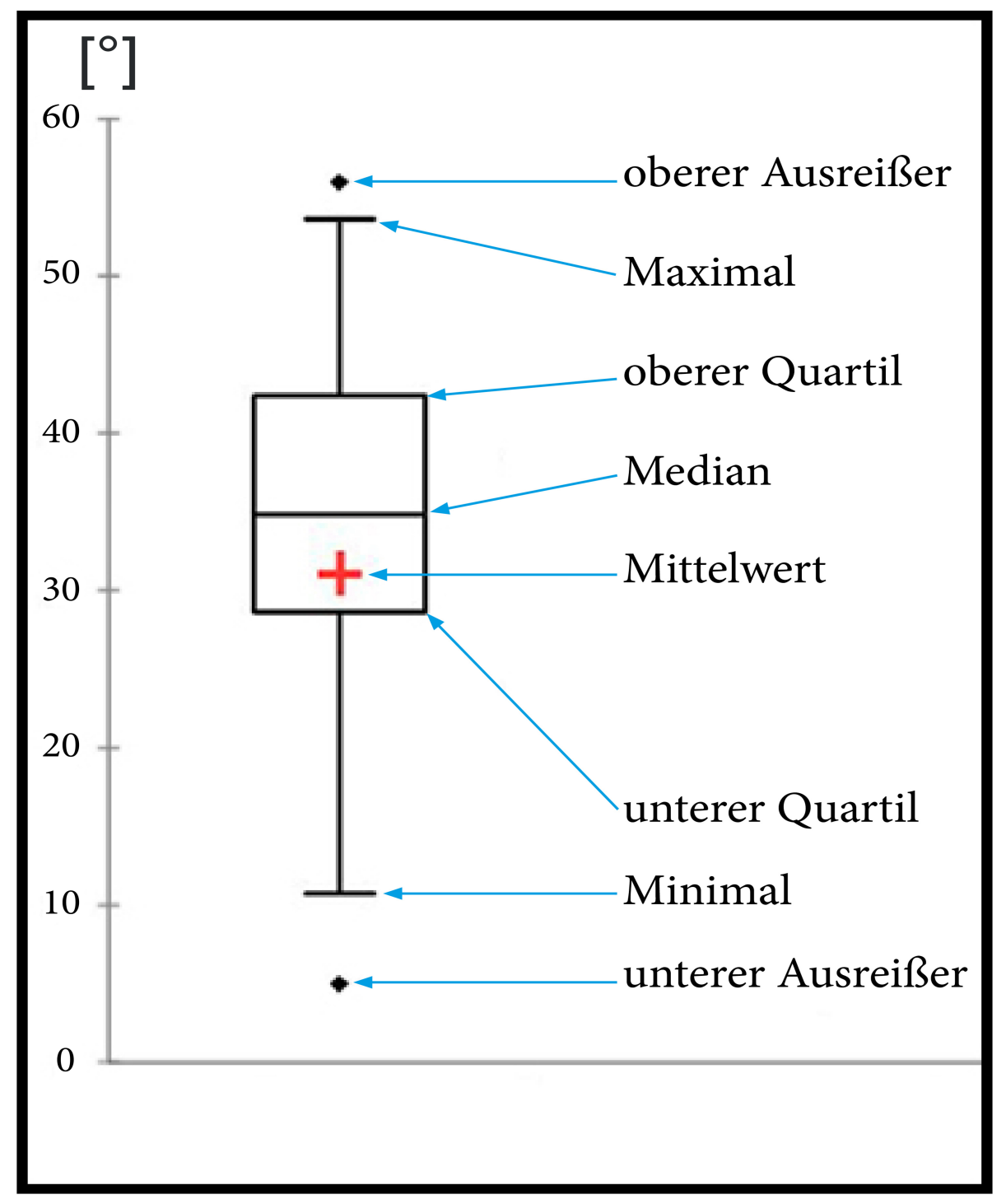

Abbildung 26: Erläuterung Whisker-Box-Plot 


\subsection{Mittelwerte}

Die indirekte und die direkte Anlegetechnik führten zu insgesamten Mittelwerten des Bennett-Winkels (BA) und der Kondylenbahnneigung $(\mathrm{HCI})$ von $8,0^{\circ}\left( \pm 4,6^{\circ}\right)$ sowie $33,1^{\circ}\left( \pm 12,2^{\circ}\right)$. Die mittleren Differenzen des BA und HCI zwischen den wiederholten Messungen mit Entfernung des Geräts betrugen über alle ProbandInnen und Anlegetechniken hinweg $1,10^{\circ}\left(0,6^{\circ}-4,7^{\circ}\right)$ sowie $2,19^{\circ}\left(0,3^{\circ}-6,6^{\circ}\right)$. Die mittleren Werte für den BA und HCI für die direkte Anlegetechnik lagen bei $8,2^{\circ}\left( \pm 4,8^{\circ}\right)$ sowie $34,2^{\circ}\left( \pm 12,3^{\circ}\right)$. Die indirekte Anlegetechnik führte zu Mittelwerten für BA und HCI von $7,8^{\circ}\left( \pm 4,5^{\circ}\right)$ und $32,1^{\circ}\left( \pm 12,1^{\circ}\right)$. Die mittleren Differenzen für den BA und HCI in der Gruppe mit ausreichender Retentionsfläche waren $0,9^{\circ}\left( \pm 1,0^{\circ}\right)$ sowie $2,1^{\circ}\left( \pm 1,3^{\circ}\right)$ und in der Gruppe mit einer verringerten Retentionsfläche $2,3^{\circ}\left( \pm 1,3^{\circ}\right)$ bzw. $1,3^{\circ}\left( \pm 1,1^{\circ}\right)$. Eine genaue grafische Gegenüberstellung der beiden Techniken und den zugehörigen Messwerten ist in Abbildung 27 aufgeführt.

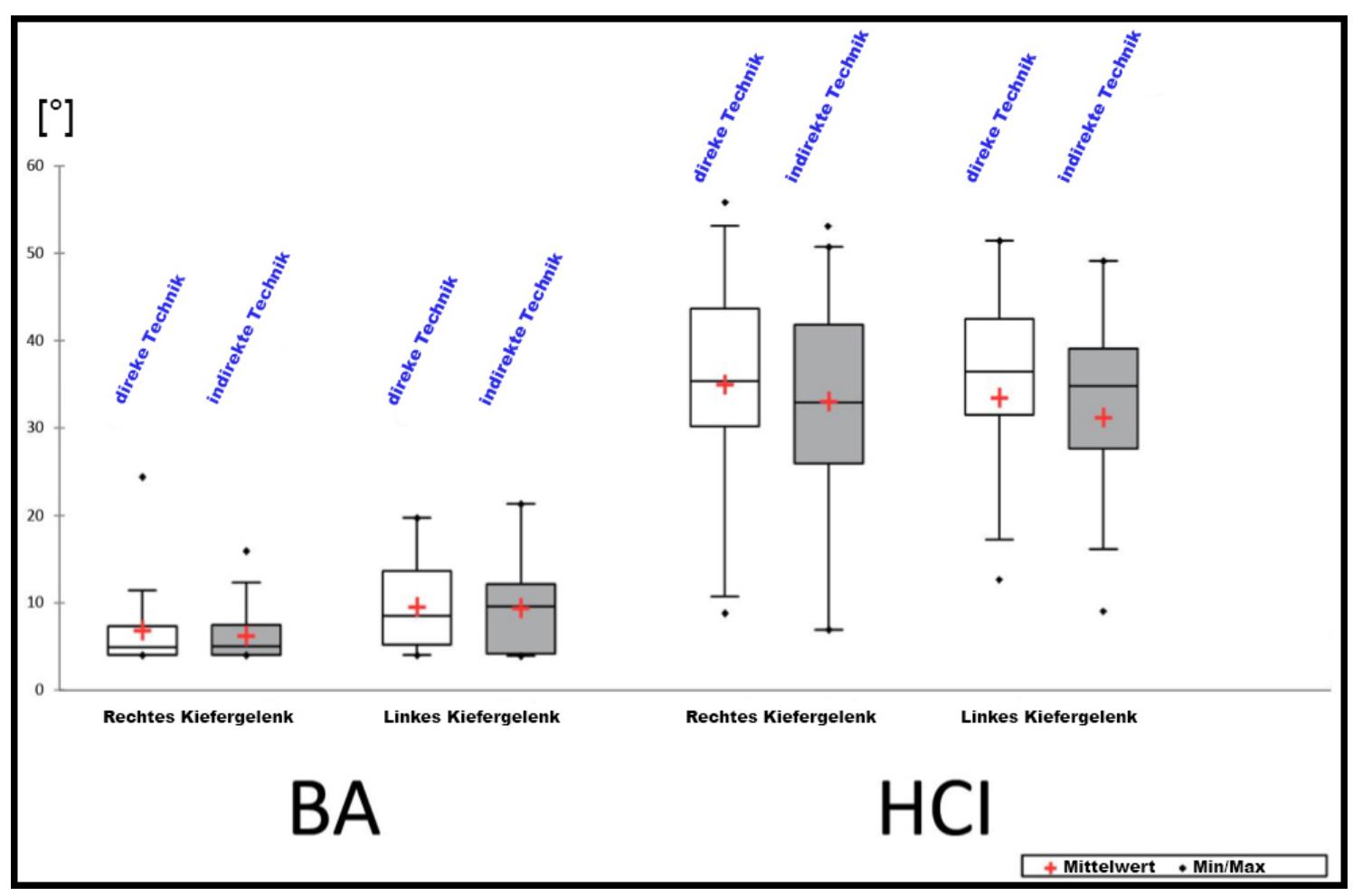

Abbildung 27: Grafische Darstellung aller Messwerte :

$\mathrm{BA}=$ Bennett-Winkel,$\quad \mathrm{HCI}=$ Kondylenbahnneigungswinkel 


\section{$3.3 \quad$ Test-Retest}

Die Messungen mit der größten Inter-Session-Differenz wurden gewählt, um die TestRetest-Reliabilität zu überprüfen. Zur Bestimmung der Reliabilität beider Anlegetechniken wurden die Werte des BA und HCI einzeln und zusammen untersucht. Die zugehörigen Werte beider Techniken und Messparameter sind in Tabelle 3 bis Tabelle 6 aufgeführt. Die parameterbezogene Reliabilität für den BA lag bei der indirekten Anlegetechnik bei 0,75 und für die direkte Anlegetechnik bei 0,66. Die Reliabilität der Messungen des HCI lag bei der indirekten Anlegetechnik bei 0,61 und bei der direkten Anlegetechnik bei 0,71. Somit weisen die wiederholten Messungen (TestRetest) der direkten Anlegetechnik für den HCI und der indirekten Anlegetechnik für den BA eine starke positive Korrelation auf. Die Messungen für den HCI der indirekten Anlegetechnik und für den BA mittels direkter Technik zeigten eine moderate Korrelation.

Tabelle 3: BA-Messwerte mit der größten Inter-Session-Differenz zur Überprüfung der Test-Retest-Reliabilität der direkten Messtechnik

\begin{tabular}{|l|c|c|c|c|c|}
\hline & Beobachtungen & $\operatorname{Min}\left[{ }^{\circ}\right]$ & $\operatorname{Max}\left[{ }^{\circ}\right]$ & $\mathbf{M W ~}^{\circ}{ }^{\circ}$ & SD $\left.^{\circ}{ }^{\circ}\right]$ \\
\hline Messung 1 & 28 & 4,0 & 19,7 & 6,989 & 4,323 \\
\hline Messung 2 & 28 & 4,0 & 24,4 & 9,386 & 5,244 \\
\hline
\end{tabular}

Min $=$ Minimalwert,$\quad$ Max $=$ Maximalwert,$\quad M W=$ Mittelwert,$\quad S D=$ Standardabweichung

Tabelle 4: BA-Messwerte mit der größten Inter-Session-Differenz zur Überprüfung der Test-Retest-Reliabilität der indirekten Messtechnik

\begin{tabular}{|l|c|c|c|c|c|}
\hline & Beobachtungen & $\operatorname{Min}\left[{ }^{\circ}\right]^{-}$ & $\operatorname{Max}\left[{ }^{\circ}\right]$ & $\left.\mathbf{M W ~}^{\circ}{ }^{\circ}\right]$ & SD $\left.^{\circ}{ }^{\circ}\right]$ \\
\hline Messung 1 & 28 & 3,9 & 18,8 & 7,011 & 3,991 \\
\hline Messung 2 & 28 & 4,0 & 21,3 & 8,568 & 4,705 \\
\hline
\end{tabular}

Min $=$ Minimalwert, Max $=$ Maximalwert,$\quad M W=$ Mittelwert,$\quad S D=$ Standardabweichung

Tabelle 5: HCI-Messwerte mit der größten Inter-Session-Differenz zur Überprüfung der Test-Retest-Reliabilität der direkten Messtechnik

\begin{tabular}{|l|c|c|c|c|c|}
\hline & Beobachtungen & Min $\left[{ }^{\circ}\right]$ & $\operatorname{Max}\left[{ }^{\circ}\right]$ & MW $\left[{ }^{\circ}\right]$ & SD $\left.^{\circ}{ }^{\circ}\right]$ \\
\hline Messung 1 & 28 & 8,8 & 53,1 & 34,775 & 11,184 \\
\hline Messung 2 & 28 & 3,4 & 49,1 & 34,714 & 11,192 \\
\hline
\end{tabular}

Min $=$ Minimalwert, Max $=$ Maximalwert,$\quad M W=$ Mittelwert,$\quad S D=$ Standardabweichung 
Tabelle 6: HCI-Messwerte mit der größten Inter-Session-Differenz zur Überprüfung der Test-Retest-Reliabilität der indirekten Messtechnik

\begin{tabular}{|l|c|c|c|c|c|}
\hline & Beobachtungen & $\operatorname{Min}\left[^{\circ}{ }^{\circ}\right.$ & $\operatorname{Max}\left[{ }^{\circ}\right]$ & MW $\left[{ }^{\circ}\right]$ & SD $\left.^{\circ}{ }^{\circ}\right]$ \\
\hline Messung 1 & 28 & 6,9 & 49,1 & 32,500 & 10,986 \\
\hline Messung 2 & 28 & 5,0 & 50,7 & 33,171 & 10,215 \\
\hline
\end{tabular}

Min $=$ Minimalwert,$\quad$ Max $=$ Maximalwert,$\quad M W=$ Mittelwert,$\quad S D=$ Standardabweichung

\subsection{Variabilität}

Die Ergebnisse der mehrfaktoriellen ANOVA deuten darauf hin, dass die Anlegetechnik, das Geschlecht und der Tiefbiss der Probanden keinen signifikanten Einfluss auf die Variabilität des Bennett-Winkels und keinen signifikanten Einfluss auf die Variabilität des Kondylenbahnneigungswinkels hatten. Auf die Interpretation der statischen Ergebnisse zum Einfluss des Geschlechtes auf die Variabilität der ermittelten Winkel wurde in dieser Studie aufgrund der geringen Anzahl männlicher Probanden verzichtet. Die Ergebnisse der mehrfaktoriellen ANOVA sind in Tabelle 7 aufgeführt.

Tabelle 7: $\quad$ Ergebnisse der mehrfaktoriellen ANOVA

\begin{tabular}{|c|c|c|}
\hline Parameter 1 & Parameter 2 & p-Wert \\
\hline \multirow{3}{*}{ Bennett-Winkel } & Anlegetechnik & 0,56 \\
\cline { 2 - 3 } & Geschlecht & 0,59 \\
\cline { 2 - 3 } & Vertikaler Überbiss & 0,07 \\
\hline \multirow{3}{*}{ Kondylenbahnneigungswinkel } & Anlegetechnik & 0,24 \\
\cline { 2 - 3 } & Geschlecht & 0,10 \\
\cline { 2 - 3 } & Vertikaler Überbiss & 0,06 \\
\hline
\end{tabular}

Signifikanz wurde als $\mathrm{p} \leq 0,05$ definiert

\subsection{Bland-Altman-Analyse}

Die Bland-Altman-Analyse zeigte, dass bei der direkten Anlegetechnik 1,94 größere Kondylenbahnneigungswinkel und $0,37^{\circ}$ größere Bennett-Winkel gemessen wurden. Die zugehörigen Diagramme sind in Abbildung 28 und Abbildung 29 dargestellt. 


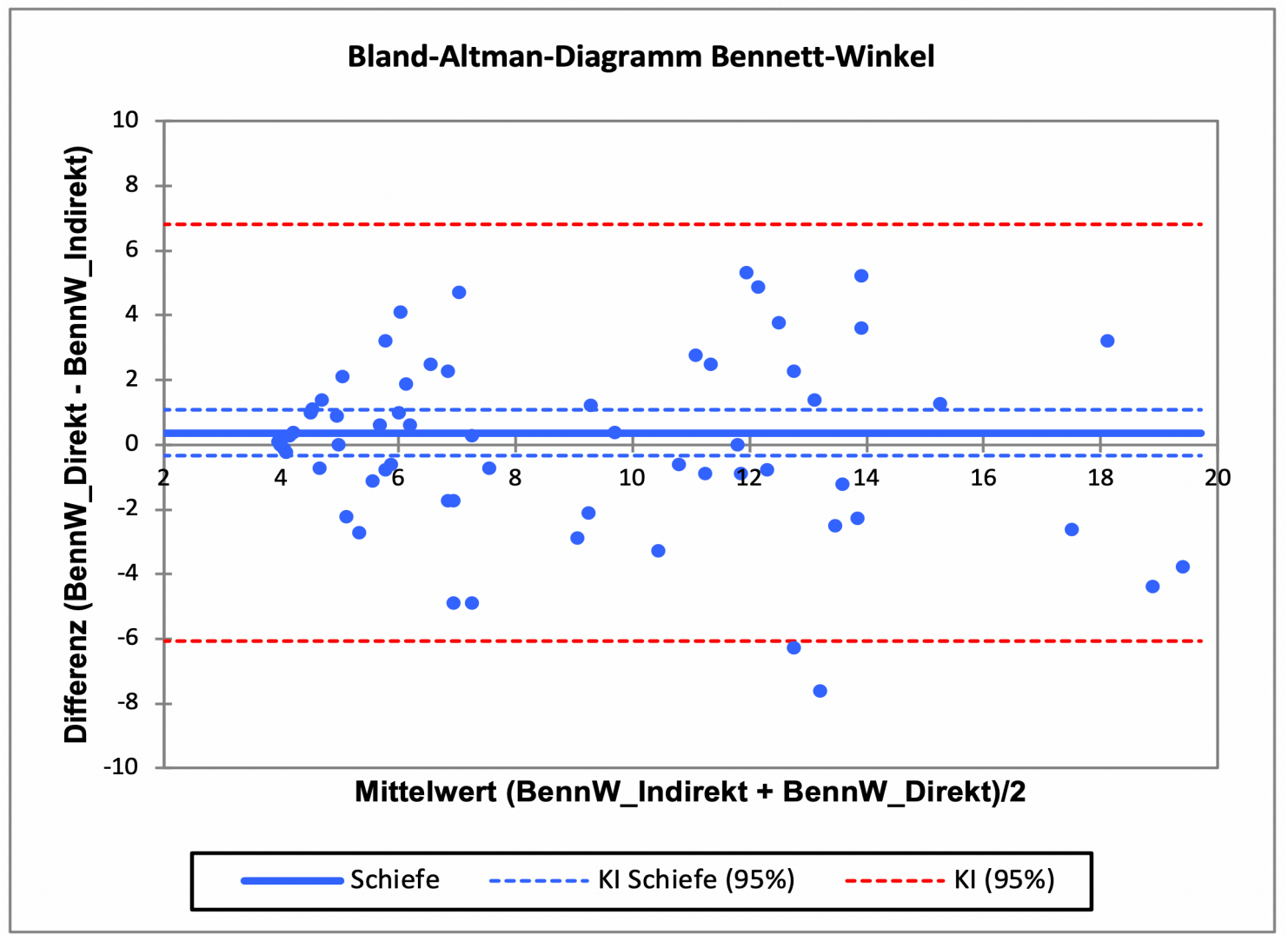

Abbildung 28: Bland-Altmann-Diagramm zum Bennett-Winkel

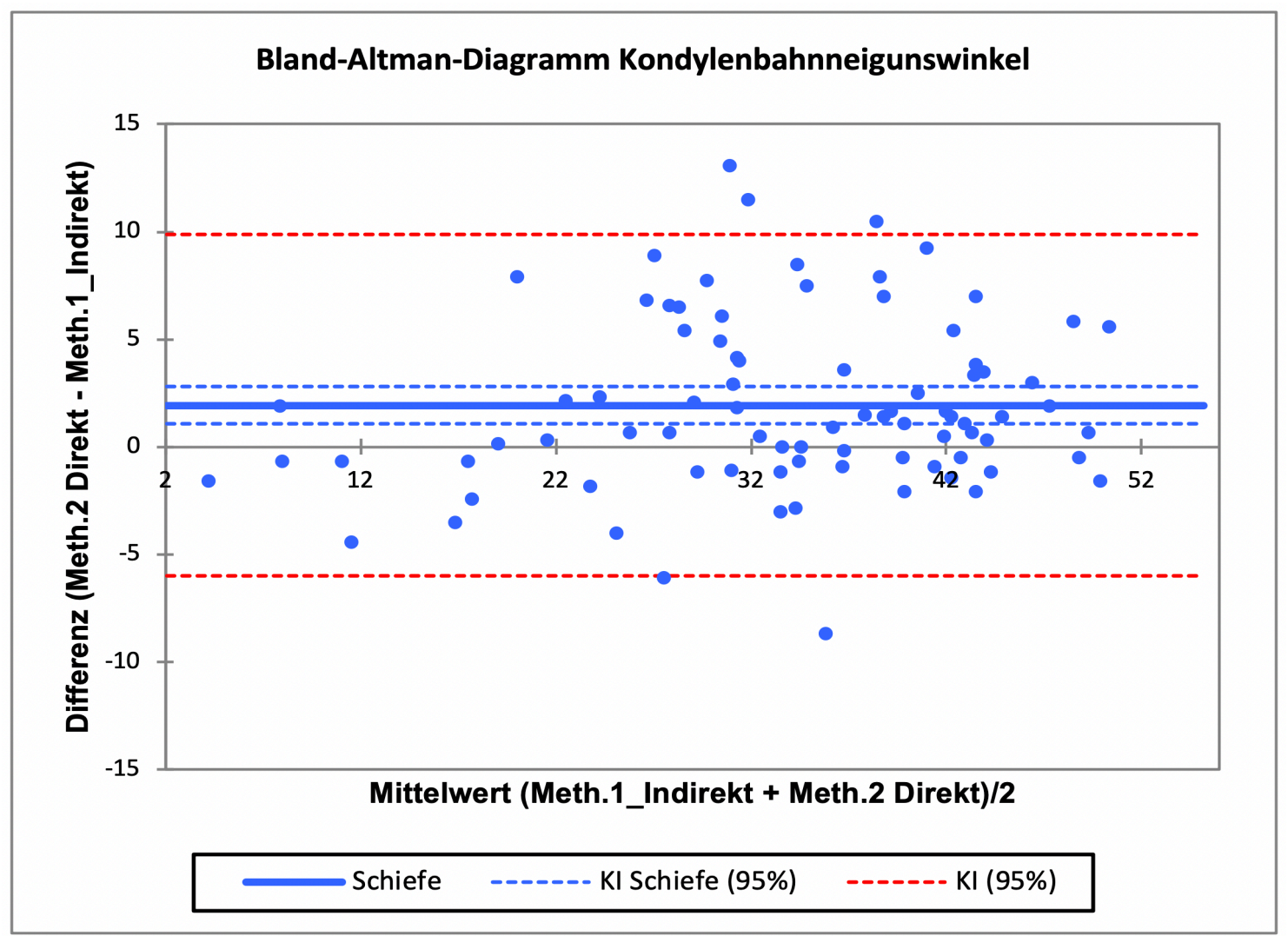

Abbildung 29: Bland-Altmann-Diagramm zum Kondylenbahnneigungswinkel 


\subsection{Klinische Beobachtungen}

Die Ergebnisse dieser Studie zeigen, dass beide Anlegetechniken objektiv übereinstimmende Messungen ermöglichen. Dennoch bestehen zwischen den beiden Anlegetechniken klinische Unterschiede, die trotz gleichwertiger Messergebnisse eine Anwendung in spezifischen Situationen erschweren können. Für beide untersuchten Anlegetechniken werden paraokklusale Löffel an die vestibulären Facetten der Unterkieferzähne befestigt. Im Rahmen der indirekten Technik wird der paraokklusale Löffel basierend auf Ober- und Unterkieferabformungen laborseitig individualisiert. Diese Individualisierung erfolgt bei der direkten Technik ohne Laboraufwand an den PatientInnen mit einem Registriermaterial. Das in dieser Studie verwendete extraharte additions-vernetzende Registriermaterial zeigte klinisch eine gute Anhaftung an der Zahnoberfläche. Jedoch wurde die klinische Anwendung bei unzureichender, vertikaler Retentionsfläche deutlich erschwert. Bei diesen ProbandInnen hat sich die direkte Fixierung des paraokklusalen Löffels mehrmals gelöst, sodass diese Messungen wiederholt werden mussten. Dieses Phänomen wurde bei der indirekten Anlegetechnik nicht beobachtet, sodass beide Methoden objektiv vergleichbare Messungen erlauben, aber die indirekte Anlegetechnik, insbesondere bei verringerter Retentionsfläche, klinisch eine bessere Handhabbarkeit aufweist. Die klinisch beobachteten Vor- und Nachteile der jeweiligen Anlegetechnik sind in Tabelle 8 aufgeführt. Aufgrund der mäßigen Probandenzahl kann die Beurteilung nur deskriptiv und nach subjektiven Eindrücken der Anwender und nicht statistisch erfolgen.

Tabelle 8: Vergleich direkte Anlegetechnik und indirekte Anlegetechnik im klinischen Alltag

\begin{tabular}{|ccc|}
\hline Direkte Technik & & Indirekte Technik \\
\hline+ & Patientenkomfort & + \\
\hline+ & Aufwand (Vorbereitung) & - \\
\hline+ & Zusätzliche Kosten & - \\
\hline- & Messdauer & + \\
\hline- & Nötige Erfahrung & + \\
\hline- & Tiefbiss & + \\
\hline
\end{tabular}

$+=$ Vorteilhaft,$\quad-=$ Nachteilhaft 


\subsection{Zusammenfassung der Ergebnisse}

Die Ergebnisse der klinischen Untersuchung zeigten, dass beide Anlegetechniken (direkt/indirekt) bezogen auf die Reliabilität der Messwerte gleichwertig sind und keine statistisch signifikanten Unterschiede vorhanden waren. Die wiederholte Erhebung von Messdaten (Test-Retest) zeigt für beide Anlegetechniken eine stark positive Relation. Dies bedeutet, dass beide Anlegetechniken aufgrund der hohen Vergleichbarkeit ihrer Messdaten gleichermaßen zur Messung des Bennett-Winkels und des Kondylenbahnneigungswinkels geeignet sind. Weiterhin wurde der Einfluss verschiedener Parameter auf die Messungen untersucht. Diese Korrelationsanalyse konnte ebenfalls keinen signifikanten Einfluss von der Retentionsfläche, dem Alter oder dem Geschlecht auf die erhobenen Messwerte finden. Messungen mittels direkter Anlegetechnik führten im Durschnitt zu 2, $1^{\circ}$ kleineren Werten für den Kondylenbahnneigungswinkel und zu 0,36 ${ }^{\circ}$ kleineren Messwerten für den Bennett-Winkel. Zusammenfassend konnte diese Studie somit zeigen, dass beide Anlegetechniken unabhängig vom Alter, Geschlecht oder vertikaler Retentionsfläche gleichwertige Messungen erlauben. 


\section{Diskussion}

\subsection{Diskussion der Methodik}

\subsubsection{Studiendesign}

Am Beispiel eines Systems zur digitalen instrumentellen Bewegungsanalyse und Artikulatorprogrammierung (ARCUSdigma II) wurde im Rahmen dieser Studie die prinzipielle Reliabilität solcher elektronischen Registriersysteme untersucht. Solche Systeme werden weltweit immer mehr eingesetzt, um die Qualität zahnärztlichrestaurativer Versorgungen zu verbessern. Leider gibt es bisher keine wissenschaftlichen Daten, die sich mit der Reliabilität solcher Systeme beschäftigen. Genau diese Verlässlichkeit bzw. formale Genauigkeit ist aber Voraussetzung für die Sinnhaftigkeit eines klinischen Einsatzes. In der Regel werden für ähnliche Studien ProbandInnen rekrutiert, die mit Hilfe der Messsysteme zu unterschiedlichen Messzeitpunkten untersucht werden. Im Anschluss erfolgt, ähnlich wie in der vorliegenden Arbeit, die statistische Auswertung der Messergebnisse. Der grundlegende Aufbau solcher Studien zur Überprüfung der Reliabilität wurde bereits mit anderen (teilweise heute technisch veralteten) Systemen durchgeführt und ist somit erprobt (Jüngling et al. 2004; Orth 2004; Kucukkeles et al. 2005; Stiesch-Scholz et al. 2006; Oberle 2007; Best 2010). Aufbauend auf dem bewährten Studiendesign untersucht diese Studie erstmalig auch den Einfluss der Anlegetechnik eines paraokklusalen Löffels auf die Reliabilität der Messungen elektronischer Registiersysteme. Weiterhin wurde in diesem Rahmen der Einfluss des Geschlechts und eines eventuellen Tiefbisses untersucht und auf die Praxistauglichkeit überprüft. Im Folgenden sollen die Kernaspekte des Studiendesigns diskutiert werden.

\subsubsection{ProbandInnen-Selektion}

Für diese Studie wurden ProbandInnen mit einem durchschnittlichen Alter von 24,9 Jahren ( \pm 2,3 Jahre) und einer Klasse-I-Verzahnung bei vollständiger Dentition gewählt. Keiner der ProbandInnen wies Symptome einer CMD auf. Das ProbandInnenkollektiv umfasste elf Frauen und drei Männer. Hierbei lag der Fokus auf einem homogenen Altersverhältnis, da die Steilheit der sagittalen Kondylenbahnneigung vom Alter der ProbandInnen beeinflusst wird (Moffett et al. 1964; Richards 1988; Touré et al. 2005). Mit voranschreitendem Alter finden verschiedene Umbauvorgänge (Remodelling) der knöchernen Strukturen des Kiefergelenks durch pathologische oder 
physiologische Belastungen, Läsionen oder entzündliche Prozesse statt (Touré et al. 2005; Jasinevicius et al. 2006). So kommt es durch regressives Remodelling mit zunehmendem Alter zur Abflachung des Tuberculum articulare, wodurch die Steilheit der Kondylenbahn verändert wird (Moffett et al. 1964). Viele Strukturen des Kiefergelenks werden zudem in Abhängigkeit vom Restzahnbestand der PatientInnen beeinflusst (Raustia et al. 1998). Weiterhin führen anatomische oder pathologische Faktoren, wie eine CMD, zu einer erhöhten Variabilität der Messwerte (Stiesch-Scholz et al. 2006). Diese Einflussfaktoren wurden durch ein sehr homogenes ProbandInnenkollektiv mit einer Klasse-I-Verzahnung bei vollständiger Dentition ohne Anzeichen einer Funktionsstörung reduziert. Die Montage der paraokklusalen Löffel wurde durch dieses ProbandInnenkollektiv, das aufgrund des geringen Alters keinerlei Zahnersatz und Attritionen aufweist, erleichtert. Eine ähnliche ProbandInnenauswahl wurde bereits in anderen Studien getroffen (Bernhardt et al. 2003; Orth 2004; Best 2010; Sójka et al. 2017; Wagner 2017). Allerdings besteht in der vorliegenden Studie mit lediglich $21,4 \%$ männlichen Probanden keine gleichmäßige Geschlechterverteilung. Dadurch sind Aussagen über den Einfluss des Geschlechts auf die Reliabilität elektronischer Registriersysteme nur eingeschränkt möglich. Die Ergebnisse müssen für sich betrachtet werden und zeigen, dass das untersuchte Testsystem verlässliche und reproduzierbare Werte liefert. Eine Interpretation und Übertragung auf die Allgemeinbevölkerung oder Patienten mit funktionellen Störungen war nicht beabsichtigt und wäre unzulässig.

\subsubsection{Referenzebenen}

In der Literatur werden unterschiedliche Referenzebenen zur Messung verschiedener Parameter der Gelenksführung mittels elektronischer Registriersysteme beschrieben (Schierz und Reissmann 2008b). Am häufigsten werden hierbei die Frankfurter Horizontale oder die Camper-Ebene als Referenzebene zusammen mit der arbiträren oder kinematischen Scharnierachse als posteriore Referenz verwendet (DGPro 2010). In der Literatur gemessene Werte beziehen sich daher immer auf eine dieser beiden Bezugsebenen (Wenz und Hellwig 2018). Das ARCUSdigma-II-System kann zusätzlich mit dem KaVo-Transfer-System (KTS) eine gerätespezifische posteriore Referenzebene verwenden (Čimić et al. 2014). Bezugssystem ist hierbei das Koordinatensystem des entsprechenden PROTAR-Artikulators, wobei die Interkondylarlinie der kinematischen oder arbiträren Achse, durch eine individuell auf die Artikulatorkondylen angepasste Verbindungslinie ersetzt wird (siehe Abbildung 30) (Wegmann 2013). Zur Kalibrierung 
des ARCUSdigma II wird mit der KTS-Bissgabel vor jeder Messung die exakte Artikulatorgeometrie auf den Schädel der ProbandInnen virtuell übertragen (Hannak 2003). Dies bietet den Vorteil, dass - anders als bei der Bestimmung der kinematischen oder arbiträren Scharnierachse - durch die KTS eine fest definierte posteriore Referenzebene festgelegt wird. Allerdings schränkt die Verwendung des KTSAchsensystems daher die Artikulatorwahl stark ein, sodass im Prinzip nur die Programmierung des KaVo-PROTAR-Artikulators möglich ist (siehe Abbildung 30). Dies liegt darin begründet, dass andere Artikulatoren stattdessen die arbiträre oder kinematische Scharnierachse als posteriore Referenzebene verwenden (DGPro 2010).

A
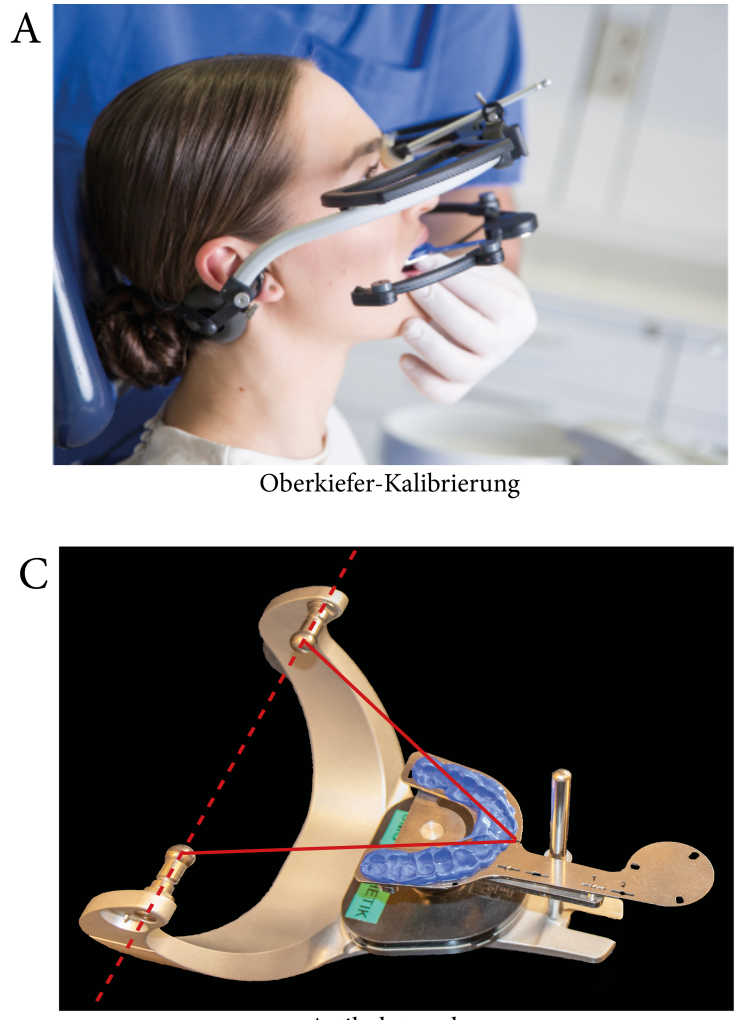

Artikulatorachse

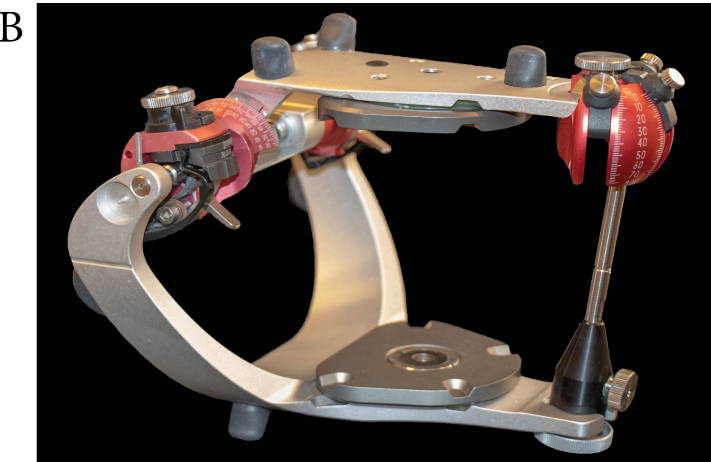

PROTAR-Artikulator

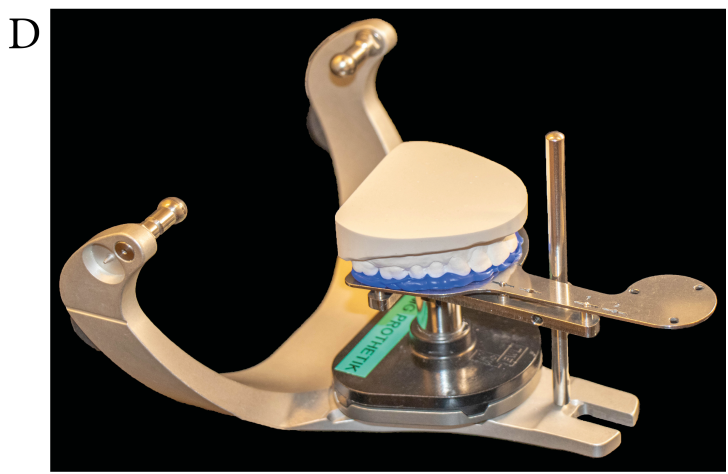

Oberkiefermodel im Artikulator

Abbildung 30:KTS-Achsensystem:

A: Mit der KTS-Bissgabel wird die Artikulatorgeometrie beim Kalibrieren übertragen,

B: Der volljustierbare Artikulator mit seinem Ober- und Unterteil,

C: KTS-Bissgabel im Artikulator-Unterteil mit exakt definierter Distanz zu den Artikulatorkondylen (Artikulatorachse),

D: Die Impressionen der KTS-Bissgabel sichern die Lage des Oberkiefers exakt zur KTS-Achse 
Die einmalige probandenspezifische Individualisierung der Oberkiefer-KTS-Bissgabel für jede(n) ProbandIn und die Verwendung dieser zur Kalibrierung vor jeder Messung gewährleisten die identische Lage der posterioren Referenzebene bei jeder Messung (siehe Abbildung 30). Um möglichst viele klinische Variablen bzw. Fehlerquellen mit potentiellem Einfluss auf die Untersuchung zu eliminieren und die Interpretation der Ergebnisse gezielt auf die Fragestellung zu fokussieren, wurde im Rahmen der Studie auf die Verwendung der arbiträren oder kinematischen Scharnierachse verzichtet und stattdessen das KTS-System eingesetzt. Auch deswegen dürfen die ermittelten Durchschnittswerte für den Bennett-Winkel und den Kondylenbahnneigungswinkel aber nicht ohne Einschränkungen mit entsprechenden Werten aus anderen klinischen Untersuchungen verglichen werden (Wegmann 2013). Im Gegensatz zu den klinisch ermittelten, absoluten Messwerten ist die erhobene Reliabilität der Untersuchungsmethode tendenziell deutlich besser mit anderen Studien vergleichbar, da die Bezugsebene hierbei von untergeordneter Bedeutung ist.

\subsubsection{Messintervall}

Die Messungen wurden je ProbandIn in drei Sitzungen im Abstand von zwei Wochen durchgeführt. Die Ergebnisse dieser Messungen wurden zur Bestimmung der Reliabilität verwendet. Die untersuchten Zielparameter (Bennett-Winkel und Kondylenbahnneigung) sind konstant und können sich im Untersuchungszeitraum nicht ändern. Eventuelle Abweichungen der Werte zu den unterschiedlichen Messzeitpunkten könnten prinzipiell auf zwei Ursachen zurückgeführt werden. Neben der möglicherweise schlechten Reliabilität des Registriersystems könnte das komplexe klinische Prozedere (z. B. das Anlegen der Oberkiefer- und Unterkieferbögen) zu Abweichungen der Messwerte führen. In beiden Fällen wäre die klinische Anwendung des Verfahrens zur Verbesserung prothetischer Restaurationen ungeeignet und ein eventueller Einsatz in der Patientenversorgung nicht sinnvoll.

Das angewandte Messintervall zur Untersuchung der Inter-Session-Reliabilität entspricht weitestgehend den Intervallen anderen Studien (Jüngling et al. 2004; Kucukkeles et al. 2005; Oberle 2007; Best 2010; Wagner 2017). Es erfolgte für jede Anlegetechnik eine Messung je Sitzung. Anders als in anderen Studien wurden keine Doppelmessungen innerhalb einer Sitzung durchgeführt (Bernhardt et al. 2003; Schierz et al. 2014; 2019; Wagner 2017). Daher konnte im Rahmen dieser Studie lediglich die Inter-Session- 
Reliabilität und nicht die Intra-Session-Reliabilität bestimmt werden. Allgemeinhin ist jedoch bekannt, dass mehrfach hintereinander ausgeführte Unterkieferbewegungen zu unterschiedlichen Bewegungsbahnen mit Messsystem-abhängigen, mittelwertigen Abweichung der Kondylenbahnneigung von $0,8^{\circ}$ bis zu 3, $1^{\circ}$ führen können (Miura et al. 1992; Baulig und Reiber 1995). Insgesamt ist jedoch die Inter-Session-Reliabilität in der Regel geringer als die Intra-Session-Reliabilität (Wagner 2017), sodass die Erhebung der Intra-Session-Reliabilität im Rahmen dieser Studie aufgrund der hohen Inter-SessionReliabilität keinen wissenschaftlichen Mehrwert geboten hat.

\subsubsection{Einflüsse auf die Messgenauigkeit}

Für beide Anlegetechniken sind Fehler hinsichtlich Montage und Anwendung des ARCUSdigma II trotz strenger Einhaltung der Herstellervorgaben nicht auszuschließen. Die wichtigsten Fehlerquellen, die häufig in der Literatur beschrieben werden, sind Störfrequenzen, Divergenzen zwischen dem Sender- und Empfängerbogen, schnelle bzw. ruckartige Bewegungen während des Messvorgangs, Positionsveränderungen des Empfängersystems oder Lockerung des paraokklusalen Löffels und Veränderung der Vertikaldimension (KaVo Dental GmbH 2008; Schierz und Reissmann 2008b; Ahlers und Jakstat 2009; Edelhoff 2015; Schierz et al. 2019). Die Gebrauchsanleitung des Systems gibt an, dass das ARCUSdigma-II-System durch Störfrequenzen, zum Beispiel von anderen elektronischen Geräten, beeinflusst werden kann (KaVo Dental GmbH 2008). Daher sollten möglichst alle Störfaktoren, wie drahtlose Sensoren anderer medizinischer Geräte, im Behandlungsraum vermieden werden (Fuentes et al. 2017). Zur Standardisierung wurden daher alle Messungen dieser Studie immer in derselben zahnärztlichen Behandlungseinheit der Poliklinik für Zahnärztliche Prothetik der Universitätsmedizin Göttingen durchgeführt. Somit wurden Veränderungen zwischen den Messungen weitestgehend ausgeschlossen. Weiterhin können Veränderungen der Distanz zwischen Sender- und Empfängerbogen während der Registrierung auftreten und zur Verfälschung der Aufzeichnungen führen. Ursächlich sind die Fehllokalisierung der Referenzachse oder die Veränderung dieser durch unterschiedliche Verläufe der Bewegungsbahnen beider Kondylen. Ohlrogge und Mack (1988) konnten beobachten, dass flache Bahnen noch flacher und steile Bahnen steiler registriert werden, wenn eine unterschiedliche Neigung zwischen der linken und rechten Protrusionsbahn vorliegt. Diese Vergrößerungseffekte wurden auch bei der Ermittlung des Bennett-Winkels durch die Registrierung der Mediotrusionsbewegungen beobachtet (Klett 1982b; 1983). 
Schnelle oder ruckartige Bewegungen während des Messvorgangs können durch Nachschwingen der Messbögen als scheinbare Bewegungen des Kondylus fehlinterpretiert werden (Klamt et al. 1990; Cousin J 1992). Neben diesen unkontrollierten Bewegungen können Positionsveränderungen des Empfängersystems oder die Lockerung des paraokklusalen Löffels zu Schwingungsartefakten führen, die zwangsläufig einen Neustart der Messungen erfordern (Edelhoff 2015). Dementsprechend fanden Ahlers und Jakstat heraus, dass die Reliabilität der Ergebnisse durch die stabile Montage der Bögen am Kopf und am Unterkiefer der Probanden sowie die Körperhaltung während der Messung beeinflusst werden kann (Ahlers und Jakstat 2009). Zusätzlich werden die Messungen durch das Gewicht des elektronischen Registriersystems beeinflusst (Lotzmann und Kobes 1988). Dabei scheint ein Gewicht von größer oder gleich $100 \mathrm{~g}$ des Empfängerbogens die Messung maßgeblich zu beeinflussen und somit zu fehlerhaften Daten zu führen (Lotzmann und Kobes 1988; Klamt et al. 1990). Demnach kann bei Verwendung des ca. $40 \mathrm{~g}$ schweren, paraokklusal befestigten Senderbogens des ARCUSdigma II, eine nennenswerte Beeinflussung der Unterkieferbeweglichkeit ausgeschlossen werden. Da zahlreiche Einflussfaktoren, wie Bewegungen der ProbandInnen, einen Einfluss auf die Messgenauigkeit und somit bei wiederholten Messungen auf die Reliabilität haben, wurden in dieser Studie alle Messungen in aufrechter, abgestützter Sitzposition auf demselben Behandlungsstuhl durchgeführt.

Die zentrale Voraussetzung zur Messung von Bewegungsbahnen in wissenschaftlichen Arbeiten ist ein standardisierter Ausgangspunkt (Ettlin und Gallo 2018). Aus diesem Grund ist der Startpunkt für alle Unterkieferbewegungen in dieser und den meisten anderen Untersuchungen die maximale Interkuspidation, da diese die einzig relativ sicher reproduzierbare Unterkieferlage ist (Fuhr et al. 1971). Dabei sollte eine okklusale Bisssperrung und somit die Erhöhung der Vertikaldimension vermieden werden, da dies eine sofortige Lageveränderung des Kondylus zur Folge hat (Čimić et al. 2016a). Bewegungen aus der habituellen Okklusion (IKP) stellen stets kombinierte DrehGleitbewegungen dar (Lindauer et al. 1995; Chen 1998). Durch die Erhöhung der Vertikaldimension werden die Kondylen aus ihrer IKP-Ausgangsposition verdrängt und infolgedessen wird ein Anteil der initialen Rotationsbewegung schon vor Messbeginn vorgenommen (Wagner 2017). Ursächlich hierfür können beispielweise bei der Befestigung des Empfängerbogens übersehene Überschüsse des Befestigungsmaterials sein. Die Auswirkung solcher okklusalen Interferenzen auf die Messwerte schwanken in 
der Literatur stark und werden unterschiedlich beurteilt (Posselt 1952; 1958; 2001; Körber 1971; Utz et al. 1987; Engelskirchen C 1998). Weiterhin kann die periokklusale Befestigung anderer Systeme durch die Bedeckung der Zahnflächen zur Erhöhung der Vertikaldimension führen (Ahlers 2009). In einer aktuellen Dissertation zum Einfluss der Bisssperrung auf die Steilheit des Kondylenbahnneigungswinkels konnte gezeigt werden, dass bei neutraler Vertikaldimension signifikant steilere Bahnen gemessen wurden als bei einer erhöhten Vertikaldimension (Wagner 2017). Um unterschiedliche Vertikaldimensionen als Einflussfaktor auf die Reliabilität auszuschließen, wurden in dieser Studie nur paraokklusale Befestigungstechniken untersucht und die Oberkieferzähne bei der Adaption mit Wachs bedeckt, um die Okklusalflächen vor Überschüssen des jeweiligen Befestigungsmaterials zu schützen (siehe Abbildung 15 und Abbildung 19). Zur Messung wurden die Wachsplatten entfernt und die statische und dynamische Okklusion vor jeder Messung akribisch auf Störfaktoren überprüft.

\subsubsection{Statistik}

In der Medizin und in der Zahnmedizin werden häufig zwei verschiedene diagnostische Methoden im Hinblick auf ihre Messgenauigkeit untersucht. Theoretisch werden die ermittelten Werte beider Techniken durch Messfehler in ungewissem Umfang verfälscht. Allerdings ist im Rahmen des Methodenvergleichs nicht bekannt, welches der beiden Verfahren die Ergebnisse liefert, die näher an den tatsächlichen Werten liegen. Wie von Grouven und seinen Mitarbeitern (2007) beschrieben, ist es für den Methodenvergleich von entscheidender Bedeutung, sowohl die durchschnittliche Abweichung als auch die Streuung der Abweichung individueller Messungen zu vergleichen. Die Berechnung von Korrelationskoeffizienten zweier Methoden ist daher nicht zielführend, da das Ziel des Methodenvergleichs in der Quantifizierung des Unterschiedes liegt und nicht im Testen einer statistischen Hypothese (Altman und Bland 1983; Grouven et al. 2007). Auf Basis dieser theoretischen Überlegungen ist die statistische Berechnung eines einzelnen Messwertes, der die Übereinstimmung zweier Messmethoden beschreibt, klinisch wenig bis nicht aussagekräftig (Bland und Altman 1986; Dewitte et al. 2002). Im Rahmen der vorliegenden Studie wurde daher die Bland-Altman-Analyse zum Vergleich des direkten und des indirekten Anlegeverfahrens angewendet. 


\subsubsection{Definition des ,Tiefbisses“}

Die vertikale Frontzahnstufe beschreibt den frontalen Überbiss (Overbite) der Schneidekanten der Oberkiefer- und Unterkieferzähne im Frontzahnbereich bei der maximalen Interkuspidation (siehe Abbildung 31) (Weber 2017). Der Normbereich wird in der Literatur mit $2 \mathrm{~mm}$ bis $3 \mathrm{~mm}$ angegeben (Diedrich et al. 2000; Nötzel und Schultz 2001). Ein Overbite von mehr als $3 \mathrm{~mm}$ wird als dentaler Tiefbiss bezeichnet (Weber 2017). Der Tiefbiss beschreibt somit nicht die in IKP sichtbare UnterkieferfrontzahnFacette. In dieser Studie wird jedoch der Begriff Tiefbiss für die noch sichtbare und somit für die Fixierung des Löffels noch verfügbare Retentionsfläche angewendet (siehe Abbildung 32). Diese beiden Parameter beeinflussen sich jedoch nur bedingt, da die Retentionsfläche durch verschiedene Faktoren, wie die individuelle Zahnlänge, Zahnstellung, Abrasionen, Attritionen, parodontale Erkrankungen, Elongationen, u.a., ausreichend verkleinert oder vergrößert sein kann. Aufgrund des jungen und funktionell unauffälligen ProbandInnenkollektivs in der vorliegenden Studie sind diese Einflussfaktoren vermutlich jedoch $\mathrm{zu}$ vernachlässigen und somit liegt bei den untersuchten Patienten eher ein starker Zusammenhang zwischen einem möglicherweise vorhandenem Tiefbiss und der Retentionsfläche an den Zähnen vor. Die Zuordnung in die Gruppe „Tiefbiss“ erfolgte bei einer Retentionsfläche in IKP von weniger als $4 \mathrm{~mm}$. Ursächlich für diese Begriffsfindung ist eine vorherige Studie, in der von Fixierungsproblemen des paraokklusalen Löffels in Abhängigkeit zum tiefen Biss berichtet wurde (Edelhoff 2015). Daher sollte in dieser Studie der Einfluss der Retentionsfläche auf die Reliabilität untersucht werden.

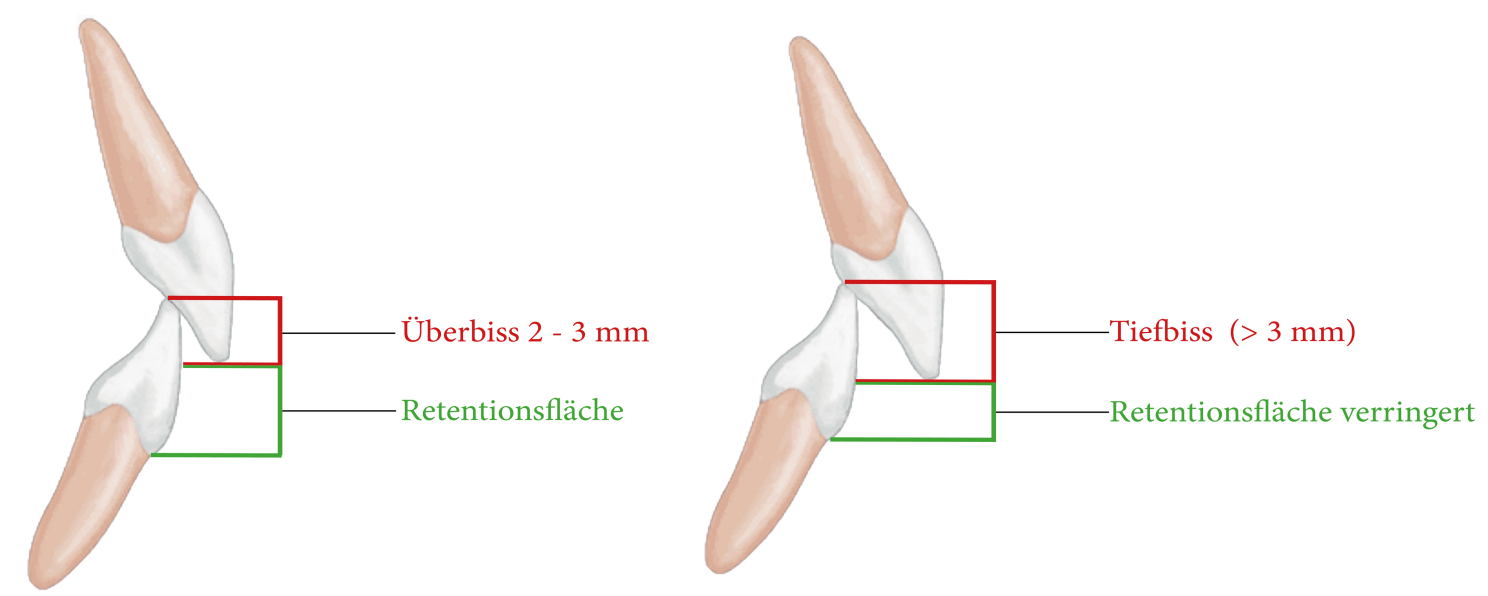

Abbildung 31: Tiefbiss und die daraus resultierende Reduktion der Retentionsfläche 


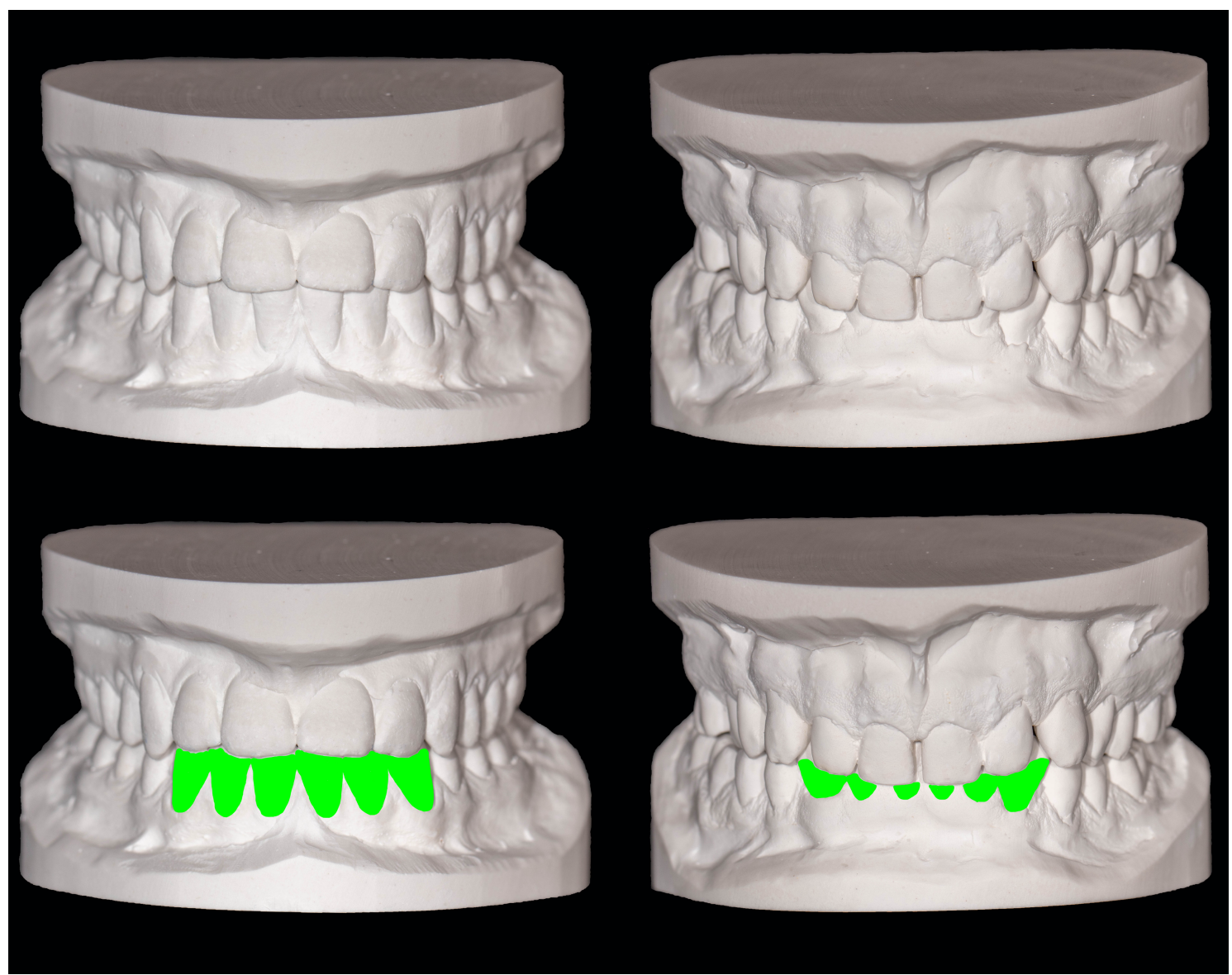

Abbildung 32: Modelle in IKP:

Links: Überbiss $2 \mathrm{~mm}$ mit ausreichend sichtbarer Retentionsfläche (grün) Rechts: Tiefbiss $7 \mathrm{~mm}$ mit deutlich verringerter Retentionsfläche (grün)

\subsection{Diskussion der Ergebnisse}

\subsubsection{Kondylenbahnneigungswinkel und Bennett-Winkel}

\subsubsection{Kondylenbahnneigungswinkel}

In dieser Studie wurden Durchschnittswerte für den Kondylenbahnneigungswinkel mit der direkten Anlegetechnik von $34,2^{\circ} \pm 12,3^{\circ}$ und mit der indirekten Technik von $32,1^{\circ} \pm 12,1^{\circ}$ gemessen. Mit anderen Registriersystemen wurden in der Literatur Durchschnittswerte für den Kondylenbahnneigungswinkel - je nach gewählter Schädelbezugsebene - von $31^{\circ}$ bis $49^{\circ}$ gemessen (siehe Tabelle 1, Kapitel 1.1.3.4). Daher liegen die erhobenen Daten im Bereich bereits bekannter Messwerte. Wie bereits im Abschnitt 4.1.3 beschrieben ist ein Vergleich dieser Messwerte jedoch nur eingeschränkt möglich, da die gewählte Referenzebene systemspezifisch ist und nicht auf anatomischen Gegebenheiten beruht. Ein direkter Vergleich mit anderen ProbandInnenkollektiven (CMD-Patienten, Allgemeinbevölkerung, etc.) unter Verwendung der entsprechenden 
Untersuchungsmethoden wäre hochinteressant, solche Studien liegen aber leider noch nicht vor, was eventuell an dem hohen instrumentellen Aufwand liegen könnte.

\subsubsection{Bennett-Winkel}

Die direkte Anlegetechnik führte zu Mittelwerten für die Bennett-Winkel von $8,2^{\circ} \pm 4,8^{\circ}$ und die indirekte Anlegetechnik führte zu Mittelwerten von $7,8^{\circ} \pm 4,5^{\circ}$. Diese Messwerte entsprechen trotz der besprochenen eingeschränkten Vergleichbarkeit den Werten der Literatur (Tabelle 2). In einer aktuellen Studie hat Čimić et al. (2016b) mit Hilfe des ARCUSdigma II sowie der KTS-Referenzebene mit einem Mittelwert von $8^{\circ} \mathrm{sehr}$ ähnliche Messwerte wie in der vorliegenden Arbeit erhoben. Die Ergebnisse dieser Studie liegen somit im erwarteten Bereich. Eine Besonderheit ist das Auftreten signifikanter Seitendifferenzen der intraindividuellen Bennett-Winkel. Dieses Phänomen wurde bereits in anderen Untersuchungen beobachtet (Theusner et al. 1993; Čimić et al. 2016b; Kordaß et al. 2019). Die Ursache hierfür kann in der Gelenkphysiologie, Gelenkanatomie und insbesondere in der Zahnführung bei Laterotrusionsbewegungen liegen (Utz et al. 1988; Bhawsar et al. 2015; Oancea et al. 2018). Zudem können die Seitendifferenzen der Bennett-Winkel als Aspekt der Lateralität des Kauorgans interpretiert werden, also als Folge einer habituell bevorzugten unilateralen Kautätigkeit. Ursächlich könnten hierbei einseitige Attritionserscheinungen bei unilateraler Kautätigkeit sein, die zu einer veränderten dentalen Führung führen könnten (Bourdiol und Mioche 2000). Zur abschließenden Klärung von diesen Ergebnissen sind Folgestudien mit einer höheren Anzahl von Probanden erforderlich.

\subsubsection{Reliabilität}

Im Rahmen dieser Studie wurde die Reliabilität der Messungen statistisch mit Hilfe eines Test-Retests ermittelt. Hierzu wurden jeweils die Messungen mit der größten InterSession-Differenz gewählt. Der Vergleich dieser wiederholten Messungen zeigte bei beiden Anlegetechniken eine starke positive Relation und somit eine hohe Reliabilität. Die Werte für die Inter-Session-Reliabilität, die in dieser Studie erhoben wurden, scheinen im oberen Bereich der bereits für andere Systeme ermittelten Werte zu liegen (Bernhardt et al. 2003; Wagner 2017). Die vergleichende Analyse wiederholter Messungen ergab in dieser Studie im Mittel Standardabweichungen von 2,19 ${ }^{\circ}$ für den $\mathrm{HCI}$ und $1,10^{\circ}$ für den BA. Diese geringen Abweichungen sind klinisch vernachlässigbar, da in der aktuellen Literatur Diskrepanzen von $3^{\circ}$ bis $4^{\circ}$ für den HCI als klinisch 
akzeptabel eingestuft werden und Abweichungen unter $3^{\circ}$ beim BA zur Herstellung von adäquatem Zahnersatz suffizient erscheinen (Beard et al. 1986; Celar und Tamaki 2002). Daher kann entsprechend vorhergehender Studien geschlussfolgert werden, dass das ARCUSdigma-II-System ein zuverlässiges Registriersystem ist (Ohlendorf et al. 2012; 2015; Edelhoff 2015; Wagner 2017). Axiographische Verfahren dienen unter anderem der graphisch-elektronischen Vermessung der Bewegungsbahnen des Kondylus für die individuelle Programmierung von volljustierbaren Artikulatoren. Die Anwendung elektronischer Verfahren gilt hierbei bereits seit längerer Zeit als wissenschaftlich anerkanntes Verfahren (Bumann und Lotzmann 2000). Die Vorteile der Axiographie bei der Herstellung von partiellem und totalem Zahnersatz sowie von Aufbissschienen wurden im Kapitel 1.1.5 ausführlich beschrieben. Die Ergebnisse dieser Studie und Untersuchungen zum Vorgängersystem zeigen, dass eine zuverlässige Artikulatorprogrammierung mittels ARCUSdigma möglich ist (Stiesch-Scholz et al. 2006; Kobs et al. 2007). Allerdings bezieht sich diese Untersuchung nur auf das Modul zur Artikulatorprogrammierung mittels KTS-Achse und nicht auf andere Anwendungsgebiete, wie z. B. das Modul zur Bewegungsanalyse. Somit spiegeln die vorliegenden Ergebnisse nicht alle Untersuchungsmodalitäten, die mit dem ARCUSdigma II möglich sind, wider. Nachfolgende Studien wären wünschenswert, auch um den Mehrwert der verfügbaren Methoden in der klinischen Anwendung im Sinne einer evidenzbasierten Zahnmedizin zu untermauern.

\subsubsection{Multifaktorielle Varianzanalyse}

Die Ergebnisse der multifaktoriellen Varianzanalyse ergaben keine Korrelation von Anlegetechnik, Geschlecht und Tiefbiss in Bezug auf die Reproduzierbarkeit der erhobenen Messwerte.

\subsubsection{Anlegetechnik}

$\mathrm{Zu}$ den verschiedenen Anlegetechniken elektronischer Registriersysteme bestehen in der aktuellen Literatur nur wenige Berichte und Studien (Ahlers 2009; Ahlers et al. 2015; Schierz et al. 2019). In der vorhandenen Literatur wird hauptsächlich zwischen para- und periokklusal fixierten Löffeln unterschieden, wobei paraokklusale Löffel von vestibulär und periokklusale Löffel von okklusal fixiert werden (Ahlers et al. 2015). Die nachteilige Erhöhung der Vertikaldimension bei Verwendung periokklusaler Löffel wurde bereits ausreichend diskutiert. Hinsichtlich der Messung des Kondylenbahnneigungswinkels zeigt eine aktuelle Studie, dass die Reliabilität von Messungen mit einem periokklusal- 
fixierten Löffel signifikant schlechter als bei der Verwendung von paraokklusalen Löffeln ist (Schierz et al. 2019). Die Autoren der Studie empfehlen daher die Verwendung von paraokklusalen Löffeln, gehen jedoch nicht auf die Fixationstechnik des Löffels ein. Dieser Fragestellung ist die vorliegende Studie nachgegangen und kommt zu den Schluss, dass sowohl die direkte als auch die indirekte Technik eine insgesamt hohe und im Vergleich gleichwertige Reliabilität aufweisen. Beide Methoden scheinen unter Berücksichtigung von praktischen Vor- und Nachteilen für die klinische Anwendung empfehlenswert.

\subsubsection{Geschlecht}

Die Probanden und Probandinnen dieser Studie setzen sich aus drei Männern und elf Frauen zusammen. In der statistischen Auswertung konnte kein signifikanter Einfluss des Geschlechts auf die Reliabilität der Messwerte beider Parameter festgestellt werden. Ursächlich könnte unter anderem die geringe Anzahl männlicher Probanden in der Studie sein. Die meisten anderen Studien zu der Reliabilität elektronischer Messsysteme mit ausgewogener Geschlechterverteilung und höheren ProbandInnenanzahlen haben den Einfluss des Geschlechts auf die Reliabilität nicht analysiert (Jüngling et al. 2004; Orth 2004; Kucukkeles et al. 2005; Stiesch-Scholz et al. 2006). Dahingegen konnte eine ältere Studie $\mathrm{zu}$ diesem Thema einen signifikanten Einfluss des Geschlechts auf die Unterkieferbeweglichkeit nachweisen (Szentpétery 1993). Jedoch zeigen drei aktuellere Untersuchungen, dass das Geschlecht keinen signifikanten Einfluss auf die Reliabilität der Messungen hat (Demling und Stiesch-Scholz 2007; Alshali et al. 2013; Schierz et al. 2014). Trotz der Einschränkungen der vorliegenden Arbeit sind die Ergebnisse demnach im Einklang mit diesen Studien und daher ist ein signifikanter Einfluss des Geschlechts auf die Reliabilität tendenziell eher unwahrscheinlich.

\subsubsection{Tiefbiss}

In einer vorherigen Studie wurde von der erschwerten Befestigung des paraokklusalen Löffels mit Hilfe eines Registriersilikons bei PatientInnen mit einem ausgeprägtem Tiefbiss und verringerter vestibulärer Retentionsfläche berichtet (Edelhoff 2015). Da in der aktuellen Literatur keine Hinweise auf den Einfluss der Retentionsfläche auf die Reliabilität von Messwerten, die durch elektronische Registriersysteme erhoben wurden, bestehen, sollte dies in der vorliegenden Arbeit untersucht werden. Die Ergebnisse der multifaktoriellen Varianzanalyse zeigen, dass der Einfluss einer verringerten Retentionsfläche trotz erschwerter klinischer Handhabung auf die Reliabilität der 
Messwerte statistisch nicht signifikant ist. Da der $p$-Wert bezogen auf den Kondylenbahnneigungswinkel und den Bennett-Winkel mit 0,06 bzw. 0,07 sehr nah am Signifikanzniveau liegt und die Anzahl der ProbandInnen mit jeweils sieben Personen sehr klein ist, könnten bei einem größeren ProbandInnenkollektiv signifikante Effekte auftreten. Da die klinische Anwendung der indirekten Anlegetechnik bei PatientInnen mit ausgeprägtem Tiefbiss deutlich erleichtert ist, sollte diese Fragestellung in einer zukünftigen Studie mit einer größeren ProbandInnenanzahl geklärt werden, um auch hier verlässliche Daten $\mathrm{zu}$ erhalten. Diese Fragestellung ist von besonderer klinischer Relevanz, da ein vergrößerter Overbite bei ca. 14-35 \% und ein ausgeprägter Tiefbiss mit traumatischem Einbiss bei ca. 1-4 \% in der Allgemeinbevölkerung vorliegt (Brunelle et al. 1996; Onyeaso 2004; Ahangar Atashi 2007; Gelgör et al. 2007; Beddis et al. 2014). 


\subsection{Schlussfolgerung}

Im Rahmen dieser Studie wurde ein belastbares Studiendesign zur Durchführung klinischer Studien mit dem ARCUSdigma II etabliert. In dieser Untersuchung wurde unter anderem die Reliabilität des Systems untersucht. Die Vermessung lieferte 42 gemessene Kondylenbahnneigungswerte sowie 42 Bennett-Winkelwerte sowohl für die direkte Anlegetechnik und als auch für die indirekte Anlegetechnik. Für die Untersuchung der Validität der Messungen standen somit insgesamt 84 gemessene Kondylenbahnneigungswerte und 84 Bennett-Winkelwerte zur Auswertung. Diese Anzahl war ausreichend, um die Unterschiede beider Anlegetechniken in Hinblick auf ihre Reliabilität und Validität statistisch zu bewerten. Die Ergebnisse dieser Studie zeigen, dass weder die Anlegetechnik noch Geschlecht oder Tiefbiss einen signifikanten Einfluss auf die Reliabilität hat. In Anbetracht dieser Studie und der aktuellen Literatur sind diese Effekte insbesondere in Bezug auf die Anlegetechnik und den Einfluss des Geschlechts besonders evident. Der Einfluss des Tiefbisses auf die Reliabilität ist jedoch aufgrund der Anzahl der ProbandInnen nicht hinreichend geklärt. Zur Abklärung wären umfangreichere Untersuchungen erforderlich.

Zusammenfassend zeigen die Ergebnisse, dass die direkte und indirekte Anlegetechnik eine hohe Reliabilität aufweisen und somit beide zur Artikulatorprogrammierung in der klinischen Anwendung geeignet sind. 


\section{Zusammenfassung}

Craniomandibuläre Dysfunktionen und funktionstherapeutische Prozedere rücken in den letzten Jahren immer mehr in den Fokus der zahnmedizinischen Forschung. Hierbei werden traditionelle, analoge Techniken durch neuere digitale Methoden ersetzt. Dieser Entwicklung trägt die S2k-Leitlinie „Instrumentelle zahnärztliche Funktionsanalyse“ aus dem Jahr 2016 der Deutschen Gesellschaft für Funktionsdiagnostik und -therapie (DGFDT) und der Deutschen Gesellschaft für Zahn-, Mund- und Kieferheilkunde (DGZMK) Rechnung. Laut dieser Leitlinie ist die instrumentelle Funktionsanalyse eine relevante diagnostische Untersuchung, mit der ergänzend zur klinischen Funktionsanalyse wertvolle Informationen zum Funktionsstatus des craniomandibulären Systems erhoben werden können. Somit dient die elektronische Axiographie als Teil der instrumentellen Funktionsanalyse der Diagnose, Dokumentation und Evaluation von Therapieverläufen und bei der Herstellung prothetischer Restaurationen der Ermittlung patientInnenindividueller Daten zur Artikulatorprogrammierung. In den letzten Jahren wurden verschiedene Messverfahren und -systeme entwickelt, um spezifische Unterkieferbewegungen zu erfassen, zu untersuchen und zu reproduzieren. Die digitale Datenspeicherung moderner elektronischer Systeme ermöglicht die dreidimensionale Visualisierung der erfassten Bewegungen und erlaubt die räumliche Berechnung der Bewegungsbahnen eines beliebigen Punktes. Berührungslose und gelenkfern registrierende Systeme, wie das Testsystem aus der vorliegenden Untersuchung (ARCUSdigma II), verwenden einen peri- oder paraokklusal befestigten Senderbogen sowie einen schädelbezüglich fixierten Empfängerbogen. Die paraokklusale Fixierung kann nach zahntechnischer Individualisierung des paraokklusalen Löffels (indirekte Technik) oder durch intraorale Befestigung mit Registriermaterial durchgeführt werden (direkte Technik). Sowohl für die instrumentelle Bewegungsanalyse als auch die individuelle Artikulatorprogrammierung ist die Erhebung reliabler Daten erforderlich. Allerdings hat sich bisher noch keine Untersuchung mit dem Einfluss der Anlegetechnik, dem Geschlecht oder eines Tiefbisses auf die Reliabilität der Messwerte beschäftigt. Daher wurde in dieser Studie die Reliabilität des Testsystems zur instrumentellen Funktionsanalyse bei der Artikulatorprogrammierung untersucht. Zusätzlich wurde der Einfluss des Geschlechts und des Tiefbisses überprüft. Das Ziel war es eine evidenzbasierte klinische Empfehlung geben zu können, welche Anlegetechnik eine höhere Reliabilität aufweist. 
Hierzu wurden 14 funktionell unauffällige ProbandInnen mit einer vollständigen Dentition akquiriert. Die Kiefer der ProbandInnen wurden abgeformt, um Oberkieferund Unterkiefersituationsmodelle im zahntechnischen Labor herstellen zu können. Sieben ProbandInnen wiesen eine Retentionsfläche zur Fixierung von paraokklusalen Löffeln von gleich oder weniger als $3,5 \mathrm{~mm}$ in maximaler Interkuspidation auf und wurden in die Tiefbissgruppe eingeschlossen. Für die indirekte Technik wurde für jeden ProbandIn ein paraokklusaler Löffel mit lichtpolymerisierendem Kunststoff (Impression Tray Resin LC, Henry Schein Deutschland GmbH, Langen, Deutschland) individualisiert. Ein weiterer paraokklusaler Löffel je ProbandIn wurde für die direkte Technik chairside mit einem Registriersilikon angepasst. Beide Techniken wurden angewendet, um an drei Messterminen in einem Abstand von je zwei Wochen die Unterkieferbewegungen aller ProbandInnen mit dem Artikulatorprogrammierungsmodul des ARCUSdigma II aufzuzeichnen. Als posteriore Referenzachse wurde immer das KaVo-Transfer-System gewählt. Die dafür notwendigen KTS-Löffel zur Kalibrierung des Oberkiefers wurden beim ersten Messtermin einmalig angepasst und für alle Messungen wiederverwendet. Im Anschluss erfolgte die statistische Auswertung der Messungen.

Die Ergebnisse dieser Studie zeigen, dass beide Anlegetechniken die Erfassung reproduzierbarer Daten erlauben und keine signifikanten Unterschiede hinsichtlich ihrer Messwerte und der Reliabilität aufweisen. Die multifaktorielle Varianzanalyse hat gezeigt, dass weder die Anlegetechnik, das Geschlecht noch ein Tiefbiss einen signifikanten Einfluss auf die Reliabilität der Messungen hatte. Somit sind beide Anlegetechniken klinisch gleichwertig. Jedoch ist die direkte Fixierung bei ProbandInnen mit wenig Retentionsfläche praktisch nur schwerer realisierbar. Dies kann insbesondere bei einem unerfahrenen Anwender zur Lockerung des fixierten Löffels führen und somit eine Messungswiederholung erforderlich machen. Die direkte Anlegetechnik hingegen ist ohne Verlust an Messgenauigkeit und Reproduzierbarkeit klinisch flexibler und anwenderfreundlicher, da die Aufzeichnung der Unterkieferbewegungen ohne vorherige Planung und ohne Laboraufwand möglich ist. Zusammenfassend ist daher die Wahl der Befestigungsmethode lediglich abhängig von der Behandlerpräferenz. 


\section{Literaturverzeichnis}

Abdal-Hadi L (1989): The hinge axis: evaluation of current arbitrary determination methods and a proposal for a new recording method. J Prosthet Dent $\underline{62}, 463-467$

Abe S, Takasaki I, Ichikawa K, Ide Y (1993): Investigations of the run and the attachment of the lateral pterygoid muscle in Japanese. Bull Tokyo Dent Coll $\underline{34}$, $135-139$

Ahangar Atashi MH (2007): Prevalence of malocclusion in 13-15 year-old adolescents in Tabriz. J Dent Res Dent Clin Dent Prospects $\underline{1}, 13-18$

Ahlers MO: Restaurative Zahnheilkunde mit dem Artex-System: Einsatz von Gesichtsbogen, Kieferrelationsbestimmung und Artikulator zur individualisierten Therapie. DentaConcept, Hamburg 1996

Ahlers MO (2009): The attachment of a paraocclusal tray adapter to the lower arch for axiography. J CranioMand Func $\underline{1}, 241-250$

Ahlers MO, Jakstat H (2008): Identifikation funktionsgestörter Patienten. Zahnmedizin up2date $\underline{2}, 143-155$

Ahlers MO, Jakstat H (2009): Development of a computer-assisted system for modelbased condylar positi analysis (E-CPM). Int J Comput Dent 12, 223-34

Ahlers MO, Biffar R, Bumann A, Freesmeyer WB, Hugger A, Kordaß B, Klett R, Koeck B, Meyer G, Ottl P et al. (2006): Gemeinsame Stellungnahme der AFDT und DGzPW: Terminologie der Arbeitsgemeinschaft für Funktionsdiagnostik und Therapie (AFDT) und der Deutschen Gesellschaft für zahnärztliche Prothetik und Werkstoffkunde (DGzPW). Dtsch Zahnarztl Z $\underline{61}, 8-10$

Ahlers MO, Bernhardt O, Jakstat HA, Kordaß B, Türp JC, Schindler HJ, Hugger A (2015): Motion analysis of the mandible: guidelines for standardized analysis of computer-assisted recording of condylar movements. Int J Comput Dent 18, 201223

Alshali RZ, Yar R, Barclay C, Satterthwaite JD (2013): Sagittal condylar angle and gender differences: Sagittal condylar angle. J Prosthodont 22, 561-565

Altman DG, Bland JM (1983): Measurement in Medicine: The analysis of method comparison studies. The Statistician $\underline{32}, 307-317$ 
Årstad T: The capsular ligaments of the temporomandibular joint and retrusion facets of the dentition in relationship to mandibular movements. Akademisk forlag, Oslo 1954

Baulig H, Reiber T (1995): Experimental studies of the precision of the String Condylocomp LR 2. Dtsch Zahnarztl Z 므, 533-535

Beard CC, Donaldson K, Clayton JA (1986): Comparison of an electronic and a mechanical pantograph. Part I: Consistency of an electronic computerized pantograph to record articulator settings. J Prosthet Dent 55, 570-574

Beddis HP, Durey K, Alhilou A, Chan MFWY (2014): The restorative management of the deep overbite. Br Dent J 217, 509-515

Benner K-U, Fanghänel J, Kowalewski R, Kubein-Meesenburg D, Randzio J, Schlegel D: Morphologie, Funktion und Klinik des Kiefergelenks. Quintessenz Verlag, Berlin 1993

Bernhardt O, Küppers N, Rosin M, Meyer G (2003): Comparative tests of arbitrary and kinematic transverse horizontal axis recordings of mandibular movements. $\mathrm{J}$ Prosthet Dent $\underline{89}, 175-179$

Best N: Untersuchungen zur Reliabilität und Validität der dreidimensionalen Kiefergelenkfunktionsanalyse mittels Zebris WinJaw-System. Med. Diss. Jena 2010

Bewersdorff H (1967): Electrognathography. Electronic 3-dimensional measurement and registration of jaw movement. Dtsch Zahn Mund Kieferheilkd Zentralbl Gesamte $\underline{48}, 100$

Bhawsar SV, Marathe AS, Ansari SA (2015): Evaluation of Hanau's formula in determination of lateral condylar guidance: A clinical research study. J Indian Prosthodont Soc 15, 326-330

Bland JM, Altman DG (1986): Statistical methods for assessing agreement between two methods of clinical measurement. Lancet $\underline{1}, 307-310$

Bojanov V, Jordanov J, Raitschinova E (1972): Über die Parallelität der Camperschen Ebene und der Okklusionsebene. Dtsch Zahnarztl Z 27, 474-477 
Bourdiol P, Mioche L (2000): Correlations between functional and occlusal toothsurface areas and food texture during natural chewing sequences in humans. Arch Oral Biol 노, 691-699

Bowley JF, Pierce CJ (1990): Reliability and validity of a transverse horizontal axis location instrument. J Prosthet Dent $\underline{64}, 646-650$

Bräunig B: Zentrische Kondylenpositionsanalyse - Übersicht und klinische Bewertung nach Untersuchungen mittels computergestützter Messsysteme. Med. Diss. Greifswald 2012

Brewer AA, Hudson DC (1961): Application of miniaturized electronic devices to the study of tooth contact in complete dentures: A progress report. J Prosthet Dent $\underline{11}$, $62-72$

Brunelle JA, Bhat M, Lipton JA (1996): Prevalence and distribution of selected occlusal characteristics in the US population, 1988-1991. J Dent Res $\underline{75}$, 706-713

Bumann A, Lotzmann U: Funktionsdiagnostik und Therapieprinzipien (Farbatlanten der Zahnmedizin). Band 12; Georg Thieme Verlag, Stuttgart 2000

Burckhardt R (1982): Optoelektronische berührungslose Kiefergelenksbewegungsregistrierung mit linearer Messwertfassung digitaler Messwertverarbeitung Vortrag im Rahmen der Jahrestagung der AG für Funktionsdiagnostik in der DGZMK, Bad Nauheim, 1982

Burckhardt R (1991): Elektronische Registriermethoden zur Aufzeichnung der Unterkieferbewegungen. ZMK Heute 7 , 6-14

Burckhardt R, Polz M (1986): New methods in instrumentation functional diagnosis. Dent Labor (Munch) 쏘, 387-390

Carlsson GE, Magnusson T: Behandlung temporomandibulärer Funktionsstörungen in der Praxis. Quintessenz Verlag, Chicago 2000

Celar AG, Tamaki K (2002): Accuracy of recording horizontal condylar inclination and Bennett angle with the Cadiax compact. J Oral Rehabil 29, 1076-1081

Chen X (1998): The instantaneous center of rotation during human jaw opening and its significance in interpreting the functional meaning of condylar translation. Am J Phys Anthropol 106, 35-46 
Čimić S, Šimunković SK, Badel T, Dulčić N, Alajbeg I, Ćatić A (2014): Measurements of the sagittal condylar inclination: intraindividual variations. Cranio $\underline{32}, 104-109$

Čimić S, Žaja M, Kraljević S, Šimunković M, Kopić A, Ćatić null (2016a): Influence of occlusal interference on the mandibular condylar position. Acta Stomatol Croat 50 , $116-121$

Čimić S, Simunković SK, Catić A (2016b): The relationship between angle type of occlusion and recorded bennett angle values. J Prosthet Dent 115, 729-735

Cousin J: Über die Reproduzierbarkeit axiographischer Messungen mit dem StringCondylocomp LR2. Med. Diss. Münster 1992

Craddock FW, Symmons HF (1952): Evaluation of the face-bow. J Prosthet Dent $\underline{2}$, $633-642$

Dauber W (1987): Die Nachbarschaftsbeziehungen des Discus articularis des Kiefergelenks und ihre funktionelle Deutung. Schweiz Monatsschr Zahnmed 97, $427-437$

Dejak A, Gliszczynska A, Kapiczke A, Dejak B (2018): Comparison of TMJ angles based on the ARCUSdigma II examination and occlusion records in the Protar 7 articulator. Prosthodontics $\underline{68}, 73-84$

Demling A, Stiesch-Scholz M (2007): Vergleich der Reproduzierbarkeit elektronisch ermittelter Funktionsparameter bei Patienten und Probanden. Dtsch Zahnarztl Z $\underline{62}$, $162-167$

Dewitte K, Fierens C, Stöckl D, Thienpont LM (2002): Application of the BlandAltman plot for interpretation of method-comparison studies: a critical investigation of its practice. Clin Chem $\underline{48}, 799-802$

DGPro (2010): Wissenschaftliche Mitteilung der Deutschen Gesellschaft für Prothetische Zahnmedizin und Biomaterialien e. V. (DGPRo) (vormals DGZPW): Anwendung des Gesichtsbogens beim funktionsgesunden Patienten im Rahmen restaurativer Maßnahmen. Dtsch Zahnarztl Z 65, 690-694

Diedrich P, Berg R, Haunfelder D (Hrsg.): Kieferorthopädie II: Praxis der Zahnheilkunde Studienausgabe. 4. Auflage; Urban \& Fischer, München 2000

Downs WB (1948): Variations in facial relationships: Their significance in treatment and prognosis. Am J Orthod $\underline{34}, 812-840$ 
Edelhoff JM: Vergleichende Untersuchungen zur Unterkieferbewegung mittels Videosequenz- und 3D-Ultraschallanalyse. Med. Diss. Göttingen 2015

Edinger D (1990): A new ultrasound system for recording masticatory function in implant patients. Dtsch Zahnarztl Z $\underline{45}$, S7-10

Ellis E, Tharanon W, Gambrell K (1992): Accuracy of face-bow transfer: Effect on surgical prediction and postsurgical result. J Oral Maxillofac Surg 50, 562-567

Engelhardt J (1993): Die instrumentelle Funktionsanalyse im Behandlungskonzept der täglichen Praxis. Dtsch Zahnarztl Z $\underline{48}, 287-292$

Engelskirchen C: Untersuchungen zur elektronischen Vermessung der transversalen und sagittalen Unterkieferbewegungen. Med. Diss. Bonn 1998

Ettlin D, Gallo LM (Hrsg.): Das Kiefergelenk in Funktion und Dysfunktion. 1. Auflage; Georg Thieme Verlag, Stuttgart 2018

Fasold A, Kordaß B, Bumann A (2012): Zusammenhang von Belastungsvektoren und ätiologischen Faktoren im Rahmen der erweiterten Funktionsanalyse - Teil 2: Grundlagen der EAEF und Auswertung. ZWR 121, 90-92

Ferrario VF, Sforza C, Miani A, Serrao G, Tartaglia G (1996): Open-close movements in the human temporomandibular joint: Does a pure rotation around the intercondylar hinge axis exist? J Oral Rehabil 23, 401-408

Freesmeyer W, Bumann A, Fuhr K, Klett R, Koeck B, Kubein-Mesenburg D, Lotzmann U, Reich R (1992): Nomenklaturvorschläge der Arbeitsgemeinschaft für Funktionsdiagnostik innerhalb der DGZMK. Dtsch Zahnarztl Z $\underline{47}, 347-347$

Fuentes R, Arias A, Lezcano MF, Saravia D, Kuramochi G, Dias FJ (2017): Systematic standardized and individualized assessment of masticatory cycles using electromagnetic 3D articulography and computer scripts. Biomed Res Int 2017, 1-9

Fuhr K, Bansemer D, Zilz W (1971): Untersuchungen zur Reproduzierbarkeit der zentralen Okklusion. Dtsch Zahnarztl Z 26, 176-185

Gallo LM, Airoldi GB, Airoldi RL, Palla S (1997): Description of mandibular finite helical axis pathways in asymptomatic subjects. J Dent Res $\underline{76}, 704-713$

Gallo LM, Brasi M, Ernst B, Palla S (2006): Relevance of mandibular helical axis analysis in functional and dysfunctional TMJs. J Biomech $\underline{39}, 1716-1725$ 
Gärtner C: Der Virtuelle Artikulator „DentCAM“ Evaluation und Konzepte zur praktischen Realisierung. Med. Diss. Greifswald 2003

Gelgör IE, Karaman AI, Ercan E (2007): Prevalence of malocclusion among adolescents in central anatolia. Eur J Dent 1, 125-131

Gernet W, Biffar R, Beuer F, Schwenzer N, Ehrenfeld M, Klinke T, Körber E, Kordaß B, Mack F, Mundt T et al. (Hrsg.): Zahnärztliche Prothetik (Zahn-Mund-KieferHeilkunde). 3. Auflage; Georg Thieme Verlag, Stuttgart 2007

Glickman I, Pameijer JH, Roeber FW, Brion MA (1969): Functional occlusion as revealed by miniaturized radio transmitters. Dent Clin North Am 13, 667-679

Gonzalez JB, Kingery RH (1968): Evaluation of planes of reference for orienting maxillary casts on articulators. J Am Dent Assoc 76, 329-336

Goodson JM, Johansen E (1975): Analysis of human mandibular movement. Monogr Oral Sci $\underline{5}, 1-80$

Goska JR, Christensen LV (1988): Comparison of cast positions by using four facebows. J Prosthet Dent 59, 42-44

Graf H, Zander HA (1963): Tooth contact patterns in mastication. J Prosthet Dent $\underline{13}$, $1055-1066$

Griffin CJ, Hawthorn R, Harris R (1975): Anatomy and histology of the human temporomandibular joint. Monogr Oral Sci 4, 1-26

Grouven U, Bender R, Ziegler A, Lange S (2007): Vergleich von Messmethoden. Dtsch Med Wochenschr 132, 69-73

Hannak WB (2003): Gesichtsbogeneinsatz unter Berücksichtigung elektronischer Systeme. ZWR $\underline{112}, 77-80$

Hayashi T, Itoh K, Miyakawa M (1994): Determination of the kinematic axis point of the temporomandibular joint regardless of cyclic mandibular movement data. Front Med Biol Eng $\underline{6}, 199-208$

Hobo S, Mochizuki S (1983): A kinematic investigation of mandibular border movement by means of an electronic measuring system. Part I: Development of the measuring system. J Prosthet Dent 50, 368-373 
Hugger A: Gelenknahe elektronische Erfassung der Unterkieferfunktion und ihre Umsetzung in den Artikulator: klinisch-experimentelle Untersuchungen an Probanden und Patienten. Quintessenz Verlag, Berlin 2000

Hugger A, Edinger D, Stüttgen U (1996): Eine diagnostische Erweiterungssoftware zum String-Registriersystem. Dtsch Zahnarztl Z 1ㅡ, 424-428

Hugger A, Turp J, Proschel P, Strub J, Stuttgen U (2001): Tagungsbeitrage-Die

Anwendung von Gesichtsbogen in der restaurativen Therapie und

Funktionsdiagnostik--welches Evidenzniveau liegt vor? Dtsch Zahnarztl Z ㅌ6, 671675

Hugger A, Türp JC, Kerschbaum T (Hrsg.): Curriculum orale Physiologie (Curriculum). Quintessenz Verlag, Berlin 2006

Jankelson RR: Neuromuscular dental diagnosis and treatment. Ishiyaku EuroAmerica, St. Louis 1990

Jasinevicius TR, Pyle MA, Nelson S, Lalumandier JA, Kohrs KJ, Sawyer DR (2006): Relationship of degenerative changes of the temporomandibular joint (TMJ) with the angle of eminentia. J Oral Rehabil $\underline{33}, 638-645$

Jüngling N, Smolenski UC, Loth D (2004): Untersuchung zur Reliabilität und Validität der dreidimensionalen Funktionsanalyse des Kiefergelenks. Manuelle Med $\underline{42}$, $441-448$

KaVo Dental GmbH (2008): Gebrauchsanweisung ARCUSdigma II USB, SD, wireless; https://embed.widencdn.net/download/kavokerr/xs2aqrorhv/GA_ARCUSdigmaII_2 0080619_01_de.pdf?u=a0aubk; abgerufen am 09.06.2020.

KaVo Dental GmbH (2012): Instrumentelle Funktionsdiagnostik - Innovativ und praxisgerecht. ZWR $\underline{121}, 57-57$

Kim P (2020): N temporalis profundus anterior, (C) Kenhub (www.kenhub.com); https:// www.kenhub.com/de/library/anatomie/nervus-mandibularis; abgerufen am 24.01.2021

Klamt B, Nägerl H, Kubein-Meesenburg D (1990): Comparative studies of measuring methods for the six-dimensional recording of spatial mandibular movements. Dtsch Zahnarztl Z $\underline{45}, 33-35$ 
Klett R (1982a): Elektronisches Registrierverfahren für die Kiefergelenksdiagnostik. Dtsch Zahnarztl Z 37, 991-998

Klett R (1982b): Projektionsfehler bei der Winkelmessung mit Scharnierachsschreibern. Dtsch Zahnarztl Z 37, 482-486

Klett R (1983): Projection-related measurement errors in axial pantography. Dtsch Zahnarztl Z $\underline{38}, 44-46$

Knap FJ, Richardson BL, Bogstad J (1970): Study of mandibular motion in six degrees of freedom. J Dent Res $\underline{49}, 289-292$

Kober C, Sader R, Leiggener CS, Zeilhofer H-F, Berg B-I (2016): Computerassistierte Unterstützung der Diagnostik von kraniomandibulären Dysfunktionen. MKGChirurg $\underline{9}, 184-190$

Kobs G, Didziulyte A, Kirlys R, Stacevicius M (2007): Reliability of ARCUSdigma $(\mathrm{KaVo})$ in diagnosing temporomandibular joint pathology. Stomatologija $\underline{9}, 47-55$

Koeck B, Engelhardt JP, Haunfelder D, Diedrich P, Horch H-H (Hrsg.): Funktionsstörungen des Kauorgans (Praxis der Zahnheilkunde). 3. Auflage; Urban \& Schwarzenberg, München 1995

Koh Y (2020a): Kaumuskulatur von dorsal, (C Kenhub (www.kenhub.com); https:// www.kenhub.com/de/library/anatomie/musculi-pterygoidei; abgerufen am 24.01.2021

Koh Y (2020b): M. masseter, (C) Kenhub (www.kenhub.com); https://www.kenhub.com/ de/library/anatomie/musculus-masseter; abgerufen am 24.01.2021

Koh Y (2020c): Mandibula, (C) Kenhub (www.kenhub.com); https://www.kenhub.com/ de/library/anatomie/mandibula-unterkiefer; abgerufen am 24.01.2021

Koh Y (2020d): Schädel laterale Ansicht, (C Kenhub (www.kenhub.com); https://www. kenhub.com/de/library/anatomie/anatomie-des-schaedels; abgerufen am 24.01.2021

Körber E, Luckenbach A (1981): Dreidimensionale Darstellung der Bewegung einzelner Punkte eines Kiefermodelles im Artikulator. Dtsch Zahnarztl Z $\underline{\text { 36, }}$, 462466

Körber K (1971): Electronic registration of mandibular movements in normal and malfunctioning dental occlusion. Dtsch Zahnarztl Z 26, 167-176 
Kordaß B: Koppelung der Kernspintomographie des Kiefergelenkes mit computergestützten Aufzeichnungen der Kondylenbahn: neue Aspekte für die funktionsorientierte Kiefergelenkdiagnostik und -therapie. Quintessenz Verlag 1996

Kordaß B (2002): Computer-assisted instrumental functional diagnostics--state of development, possibilities, and limits. Int J Comput Dent $\underline{5}$, 249-269

Kordaß B, Mundt T (2003): Instrumentelle Funktions- und Okklusionsanalyse - stateof-the-art der diagnostischen Möglichkeiten. Teil 1: Instrumentelle Funktionsanalyse. Quintessenz 54, 1061-1077

Kordaß B, Bernhardt O, Ruge S, Ratzmann A, Hugger S, Hugger A (2019): Standard and limit values for the symmetry of articulation parameters in the temporomandibular joint area - evaluations of the associated project of the SHIP study. Int J Comput Dent 22, 353-362

Krueger GE, Schneider RL (1986): A plane of orientation with an extracranial anterior point of reference. J Prosthet Dent $\underline{56}, 56-60$

Kucukkeles N, Ozkan H, Ari-Demirkaya A, Cilingirturk AM (2005): Compatibility of mechanical and computerized axiographs: A pilot study. J Prosthet Dent $\underline{94}, 190$ 194

Leukhardt M (2010): Transferring esthetically relevant facial reference lines to the articulator Übertragung ästhetisch relevanter Bezugslinien des Gesichts in den Artikulator. J CranioMand Func 2, 311-328

Lewin A, Ramadori G: Electrognathographics: atlas of diagnostic procedures and interpretation. Quintessence Pub. Co., Chicago 1985

Lindauer SJ, Sabol G, Isaacson RJ, Davidovitch M (1995): Condylar movement and mandibular rotation during jaw opening. Am J Orthod Dentofacial Orthop 107, $573-577$

Lippert H, Deller T: Lehrbuch Anatomie. 6. Auflage; Urban \& Fischer, München 2004 Lotzmann U (1990): Betrachtungen zur Präzision und Konstanz der transversalen Scharnierachse des Unterkiefers. ZWR 99, 372-379 
Lotzmann U, Kobes L (1988): On the influence of weight and point of gravity position of the mandibular recording bow on the course of axiographic tracings. Dtsch Zahnaerztl Z $\underline{43}$, S67

Luckenbach A: Untersuchungen zur unterkieferbewegung mit hilfe eines elektronischen, computergestutzten registriersystems (ECRS). Med. Diss. Tübeingen 1983

Lynch CD, Singhrao H, Addy LD, Gilmour ASM (2010): The teaching of fixed partial dentures in undergraduate dental schools in Ireland and the United Kingdom. J Oral Rehabil 37, 908-915

Mage K, Ćelić R, Ćimić S, Dulčić N (2019): Comparison of parameters for programming adjustable dental articulators by using wax eccentric records and Arcus Digma device. Acta Stomatol Croat $\underline{53}$, 213-223

Meyer G: Praxisgerechte instrumentelle Funktionsdiagnostik. In: Hahn W (Hrsg.): Funktionslehre - aktueller Stand und praxisgerechte Umsetzung. Carl Hanser Verlag, München 1993, 59-72

Meyer G: Elektronisches Registrieren der Unterkieferbewegungen und der Kiefergelenkfunktion. In: Siebert GK (Hrsg.): Atlas der zahnärztlichen Funktionsdiagnostik. Carl Hanser Verlag, München 1996, 271-288

Meyer G, dal Ri H: Entwicklung einer dreidimensionalen Meßanordnung für die räumliche Erfassung von Unterkieferbewegungen in der Zahnheilkunde. In: Ehlers CT, Beland H (Hrsg.): Perspektiven der Informationsverarbeitung in der Medizin Kritische Synopse der Nutzung der Informatik in der Medizin (Medizinische Informatik und Statistik). Band 64; Springer Verlag, Berlin 1986, 346-348

Michelotti A, Farella M, Vollaro S, Martina R (1997): Mandibular rest position and electrical activity of the masticatory muscles. J Prosthet Dent $\underline{78}, 48-53$

Miura H, Luckenbach A, Freesmeyer W (1992): Untersuchung zur Reproduzierbarkeit des ECR-Systems. Dtsch Zahnarztl Z 47, 373-376

Moffett BC, Johnson LC, McCabe JB, Askew HC (1964): Articular remodeling in the adult human temporomandibular joint. Am J Anat 115, 119-141

Moll KJ, Moll M: Anatomie: Kurzlehrbuch zum Gegenstandskatalog. 18. Auflage; Urban \& Fischer, München 2006 
Neill D (1976): Studies of tooth contact in complete denture. Br dent J $\underline{123}, 369-378$

Nötzel F, Schultz C: Leitfaden der kieferorthopädischen Diagnostik: Analysen und Tabellen für die Praxis. Deutscher Zahnärzte Verlag, DÄV-Hanser 2001

Oancea L, Stegaroiu R, Cristache CM (2018): The influence of temporomandibular joint movement parameters on dental morphology. Ann Anat 218, 49-58

Öberg T, Carlsson GE, Fajers C-M (1971): The temporomandibular joint: A morphologic study on a human autopsy material. Acta Stomatol Croat 29, 349-389

Oberle A: Vergleichende Untersuchung von Patienten mit und ohne Myoarthropathien (MAP) mit der elektronischen Achsiographie unter Berücksichtigung der Messwiederholung. Med. Diss. Greifswald 2007

Ohlendorf D, Pusch K, Kopp S (2008): Beinlängendifferenz versus zentrische Lage des Unterkiefers. Manuelle Med $\underline{46}, 418-423$

Ohlendorf D, Heil D, Sagel L, Chung TL, Kopp S (2012): Veränderungen der Grenzbewegungen des Unterkiefers durch Folgen einer Hüftarthritis: Eine Pilotstudie. Manuelle Med 50, 493-498

Ohlendorf D, Heil L, Heil D, Kopp S (2015): Auswirkungen der Probandenmessposition auf die Lage und Bewegungen des Unterkiefers: Vergleich zwischen stehender und sitzender Ausgangsposition. Manuelle Med 53, 277-283

Ohlrogge H (1982): Elektronische Registrierung der Kiefergelenksbewegungen. Dtsch Zahnarztl Z 37, 263-264

Ohlrogge H, Mack H (1988): Verzeichnungen und Verzerrungen bei sagittalen Aufzeichnungen in der instrumentellen Funktionsanalyse. Dtsch Zahnarztl Z $\underline{4,39}$ 43

Okeson JP, de Leeuw R (2011): Differential diagnosis of temporomandibular disorders and other orofacial pain disorders. Dent Clin North Am 55, 105-120

O’Malley AM, Milosevic A (2000): Comparison of three facebow/semi-adjustable articulator systems for planning orthognathic surgery. Br J Oral Maxillofac Surg $\underline{38}, 185-190$

Onyeaso CO (2004): Prevalence of malocclusion among adolescents in Ibadan, Nigeria. Am J Orthod Dentofacial Orthop 126, 604-607 
Orth B: Aufzeichnung einiger Parameter der Kiefergelenksführung durch unterschiedliche extraorale Registriersysteme - Vergleich der Handhabung und Ergebnisse. Med. Diss. Würzburg 2004

Palla S, Gallo L, Gossi D (2003): Dynamic stereometry of the temporomandibular joint. Orthod Craniofac Res $\underline{6}, 37-47$

Piehslinger E, Bauer W, Schmiedmayer HB (1995): Computer simulation of occlusal discrepancies resulting from different mounting techniques. J Prosthet Dent $\underline{74}$, 279-283

Posselt U (1952): Studies in mobility of the human mandible. Acta Odontol Scand 10, $1-160$

Posselt U (1958): Range of movement of the mandible. J Am Dent Assoc 56, 10-13

Posselt U (2001): Terminal hinge movement of the mandible. J Prosthet Dent $\underline{86}, 2-9$

Posselt U, Odont. D (1958): Range of movement of the mandiblew. J Am Dent Assoc $\underline{56}, 10-13$

Pröbster L, Benzing U (1990): Comparison of two computer-aided recording systems: MT 1602 and Compugnath. Dtsch Zahnarztl Z $\underline{45}$, S54-8

Pröschel P (1987): An extensive classification of chewing patterns in the frontal plane. Cranio $\underline{5}, 55-63$

Pröschel P (1988): Zum Einfluss der Okklusalflächenform auf den Bewegungsablauf des Unterkiefers bei der Kaufunktion. Dtsch Zahnarztl Z $\underline{43}$, 1099-103

Pröschel P, Hofmann M (1987): Zur Problematik der Interpretation von funktionellen Unterkieferbewegungen. Teil I: Die Auswirkungen multifaktorieller Einflüsse auf die Interpretierbarkeit von Kaubewegungsaufzeichnungen. Dtsch Zahnarztl Z $\underline{42}$, 696-700

Pröschel P, Morneburg T, Hugger A, Kordass B, Ottl P, Niedermeier W, Wichmann M (2002): Articulator-related registration - a simple concept for minimizing eccentric occlusal errors in the articulator. Int J Prosthodont 15, 289-294

Pröschel PA, Maul T, Morneburg T (2000): Predicted incidence of excursive occlusal errors in common modes of articulator adjustment. Int J Prosthodont $\underline{13}, 303-310$

Ptok M (Hrsg.): Sprache Stimme Gehör: Anatomische Grundlagen: Zeitschrift für Kommunikationsstörungen. 2. Auflage; Georg Thieme Verlag, Stuttgart 2014 
Rammelsberg P: Untersuchungen über Ätiologie, Diagnose und Therapie von Diskopathien des Kiefergelenkes. Quintessenz Verlag, Berlin 1998

Raustia AM, Pirttiniemi P, Salonen MA, Pyhtinen J (1998): Effect of edentulousness on mandibular size and condyle-fossa position. J Oral Rehabil 25, 174-179

Richards LC (1988): Degenerative changes in the temporomandibular joint in two Australian Aboriginal populations. J Dent Res 67, 1529-1533

Sander FG, Schwenzer N, Ehrenfeld M, Ahlers MO, Bantleon H-P (Hrsg.):

Kieferorthopädie. 2. Auflage; Georg Thieme Verlag, Stuttgart 2011

Schierz O, Reissmann D (2008a): Die elektronische Vermessung der Gelenkbahn (Teil 1). Digital Dental News $\underline{2}, 21-27$

Schierz O, Reissmann D (2008b): Die elektronische Vermessung der Gelenkbahn (Teil 2). Digital Dental News $\underline{2}, 18-21$

Schierz O, Klinger N, Schön G, Reissmann DR (2014): The reliability of computerized condylar path angle assessment. Int J Comput Dent $\underline{17}, 35-51$

Schierz O, Wagner P, Rauch A, Reissmann DR (2019): Impact of mounting methods in computerized axiography on assessment of condylar inclination. Cranio $\underline{37}, 60-67$

Schindler H, Hugger A (2008): Welche Rolle spielt die Okklusion in der Zahnmedizin. Freie Zahnarzt 11, 48-55

Schmitter M, Giannakopoulos N, Eberhard L, Hellmann D, Kelter V, Leckel M, Schindler H (2011): Elektronische Messverfahren. Quintessenz Zahntech 37, 1212 1220

Schrader U: Untersuchung zur Meßgenauigkeit des elektronischen Meßsystems MT 1602 unter besonderer Berücksichtigung klinischer Aspekte. Med. Diss. Düsseldorf 1994

Schumacher G-H: Anatomie für Stomatologen: Kopf-Orofaziales System-Auge-Ohr. Johann Ambrosius Barth, Leipzig 1984

Schunke S (2012): Die Gesichtsbogenübertragung. Eine persönliche Betrachtung der Problematik. Quintessenz Zahntech $\underline{38}$, 2-18

Seifert D, Jerolimov V, Carek V, Ibrahimagić L (2000): Relations of reference planes for orientation of the prosthetic plane. Acta Stomatol Croat 34, 413-416 
Siebert GK (Hrsg.): Atlas der zahnärztlichen Funktionsdiagnostik. 3. Auflage; Hanser Fachbuch, München 1996

Simpson JW, Hesby RA, Pfeifer DL, Pelleu GB (1984): Arbitrary mandibular hinge axis locations. J Prosthet Dent 51, 819-822

Singh S, Rehan S, Palaskar J, Mittal S (2017): Hinge axis - location, clinical use and controversies. J Dent Res $\underline{4}, 158-161$

Sobotta J, Putz RV, Pabst R, Putz R: Atlas der Anatomie des Menschen: Band 1 Kopf, Hals, obere Extremität. 21. Auflage; Urban \& Fischer, München 2005

Sójka A, Huber J, Kaczmarek E, Hędzelek W (2017): Evaluation of mandibular movement functions using instrumental ultrasound system. J Prosthodont $\underline{26}, 123-$ 128

Sörgel W: Okklusale Differenzen bei fehlregistrierten Scharnierachsenpunkten. Med. Diss. Tübeingen 1978

Stelzenmüller W, Wiesner J (Hrsg.): Therapie von Kiefergelenkschmerzen: ein Behandlungskonzept für Zahnärzte, Kieferorthopäden und Physiotherapeuten (ZMK Praxis). 2. Auflage; Georg Thieme Verlag, Stuttgart 2010

Stiesch-Scholz M, Demling A, Rossbach A (2006): Reproducibility of jaw movements in patients with craniomandibular disorders. J Oral Rehabil $\underline{33}, 807-812$

Strub JR, Kern M, Türp JC, Witkowski S, Heydecke G, Wolfart S: Curriculum Prothetik. Band 1: Geschichte, Grundlagen, Behandlungskonzept, Vorbehandlung. 4. Auflage; Quintessenz Verlag, Berlin 2011

Sümnig W, Bartolain G, Fanghänel J (1991): Histologische Untersuchungen über die morphologischen Beziehungen des Musculus pterygoideus lateralis zum Discus articularis am menschlichen Kiefergelenk. Anat Anz 173, 279-286

Szentpétery A (1993): Clinical utility of mandibular movement ranges. J Orofac Pain $\underline{7}$, $163-168$

The Glossary of Prosthodontic Terms: Ninth edition (2017) J Prosthet Dent 117, 1-105

Theusner J, Plesh O, Curtis DA, Hutton JE (1993): Axiographic tracings of temporomandibular joint movements. J Prosthet Dent $\underline{69}$, 209-215

Touré G, Duboucher C, Vacher C (2005): Anatomical modifications of the temporomandibular joint during ageing. Surg Radiol Anat 27, 51-55 
Utz K, Lehner B, Swoboda R, Duvenbeck H, Oettershagen K (1987): Paraokklusale Axiographie: Zur Lage der individuellen terminalen Scharnierachse bei Vollbezahnten. Zahnärztl Welt 96, 706-712

Utz K, Lehner B, Swoboda R, Duvenbeck H, Oettershagen K (1988): Para-occlusal axiography: the Bennett angle during complete occlusion - a clinico-experimental study. Schweizer Monatsschrift für Zahnmedizin $=$ Revue mensuelle suisse d'odonto-stomatologie $=$ Rivista mensile svizzera di odontologia e stomatologia $\underline{98}$, 22

Utz K-H, Hugger A, Ahlers MO, Seeher W-D, Bartsch K, Feyen J, Frahn G, Gabel S, Kordaß B, Lange-Lentz B (2016): S2k-Leitlinie Instrumentelle zahnärztliche Funktionsanalyse/ S2k Guide-line Instrumental functional analysis in dentistry (AWMF Register Nr. 083-017). J CranioMand Func $\underline{8}$, 185-236

Wagner P: Steilheit und Reproduzierbarkeit der elektronischen Registrierung der Gelenk- bahnneigung in Abhängigkeit von der Befestigung am Unterkiefer. Med. Diss. Leipzig 2017

Waldeyer A, Mayet A: Anatomie des Menschen 2 - Kopf und Hals, Auge, Ohr, Gehirn, Arm, Brust. 16. Auflage; De Gruyter, Berlin 1993

Walker PM (1980): Discrepancies between arbitrary and true hinge axes. J Prosthet Dent $\underline{43}, 279-285$

Weber T: Memorix Zahnmedizin (Memorix). 5. Auflage; Georg Thieme Verlag, Stuttgart 2017

Wegmann U (2009): Das Digma2-Registriersystem. Prodental 1, 26-29

Wegmann U (2013): Wie viel Okklusion braucht der Mensch? Zahntech Mag 17, 284296

Weinberg LA (1959): The transverse hinge axis: Real or imaginary. J Prosthet Dent $\underline{9}$, $775-787$

Weinberg LA (1961): An evaluation of the face-bow mounting. J Prosthet Dent 11, 32 42

Wenz H-J, Hellwig E: Zahnärztliche Propädeutik: Einführung in die Zahnheilkunde. 14. Auflage; Deutscher Ärzteverlag, Köln 2018 
Wieckiewicz M, Zietek M, Nowakowska D, Wieckiewicz W (2014): Comparison of selected kinematic facebows applied to mandibular tracing. Biomed Res Int 2014, 818694

Wilkinson TM, Crowley CM (1994): A histologic study of retrodiscal tissues of the human temporomandibular joint in the open and closed position. J Orofac Pain $\underline{8}$, $7-17$

Wilm-Gert E: Rationelle Arbeitsabläufe in der Zahnarztpraxis (ZMK-Praxis). 1. Aufl; Georg Thieme Verlag, Stuttgart 2006

Yatabe M, Zwijnenburg A, Megens CC, Naeije M (1995): The kinematic center: A reference for condylar movements. J Dent Res $\underline{74}$, 1644-1648

Yurkstas AA, Emerson WH (1954): A study of tooth contact during mastication with artificial dentures. J Prosthet Dent $\underline{4}, 168-174$ 


\section{Danksagung}

Mein größter Dank gilt meinem Doktorvater Herrn Professor Ralf Bürgers für die Überlassung des Themas, die ausgezeichnete Unterstützung und Betreuung bei der Erstellung dieser Arbeit!

Weiterer großer Dank geht an Herrn Dr. Rasing, Herrn Dr. Krohn und Herrn Zahnarzt Hampe, die mich geduldig betreut haben und jetzt noch mit gutem Rat zur Seite stehen. Außerdem möchte ich meinen Kollegen und Freunden Frau Frach, Herrn Wiessner, Herrn Meyer und Herrn Özköklü für die engagierte Zusammenarbeit, gegenseitige Motivation und das kritische und konstruktive Lektorat dieser Arbeit danken. 